WHC-SP-1116

Revision 0

UC-630

\title{
Overheads, Safety Analysis \& Engineering FY 1995 Site Support Program Plan WBS 6.3.5
}

E. P. DiVincenzo

Date Published

September 1994

Prepared for the U.S. Department of Energy

\footnotetext{
(20) 
LEGAL DISCLAIMER

This report was prepared as an sccount of work sponsored by an agency of the United States Government. Neither the United States Government nor any agency thereof, nor any of their employees, nor any of their contractors, subcontractors or their employees, makes any warranty, express or implied, or assumes any legal liability or responsibility for the accuracy, completeness, or any third party's use or the results of such use of any information, apparatus, product, or process disclosed, or represents that its use would not infringe privately owned rights. Reference herein to any specific commercial product, process, or service by trade name, trademark, manufacturer, or otherwise, does not necessarily constitute or imply its endorsement, recommendation, or favoring by the United States Government or any agency there of or its contractors or subcontractors. The views and opinions of authors expressed herein do not necessarily state or reflect those of the United States Government or any agency thereof.

This report has been reproduced from the best available copy. Available in paper copy and microfiche.

Available to the U.S. Department of Energy and its contractors from

Office of Scientific and Technical Information

P.O. Box 62

Oak Ridge, TN 37831

(615) 576.8401

Printed in the United States of Amerca

DISCLM-3.CHP (1-91) 


\section{DISCLAIMER}

Portions of this document may be illegible in electronic image products. Images are produced from the best available original document. 


\section{RELEASE AUTHORIZATION}

Document Number: HHC-SP-1116, REV. 0

Document Title: $\quad$ Overheads, Safety Analysis \& Engineering FY 1995

Site Support Program Plan, WBS 6.3.5

Release Date: $\quad 9 / 27 / 94$

This document was reviewed following the procedures described in WHC-CM-3-4 and is:

APPROVED FOR PUBLIC RELEASE

***************

WHC Information Release Administration Specialist:

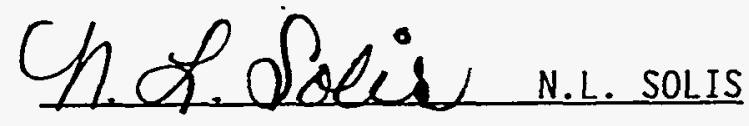

(Signature)

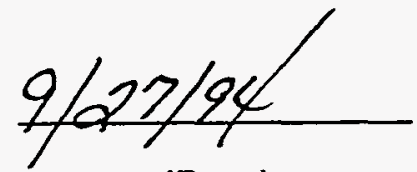

(Date) 


\section{Site Support Program Plan Approval Sheet}

\section{WBS 6.3.5 Overheads, Safety Analys is and Engineering}

FY 1995 Site Support Progran Plan

Assistant Manager-Contracting Officer's Representative
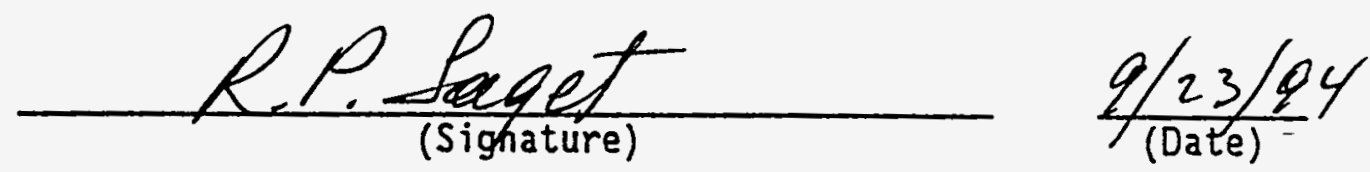

R. P. Saget
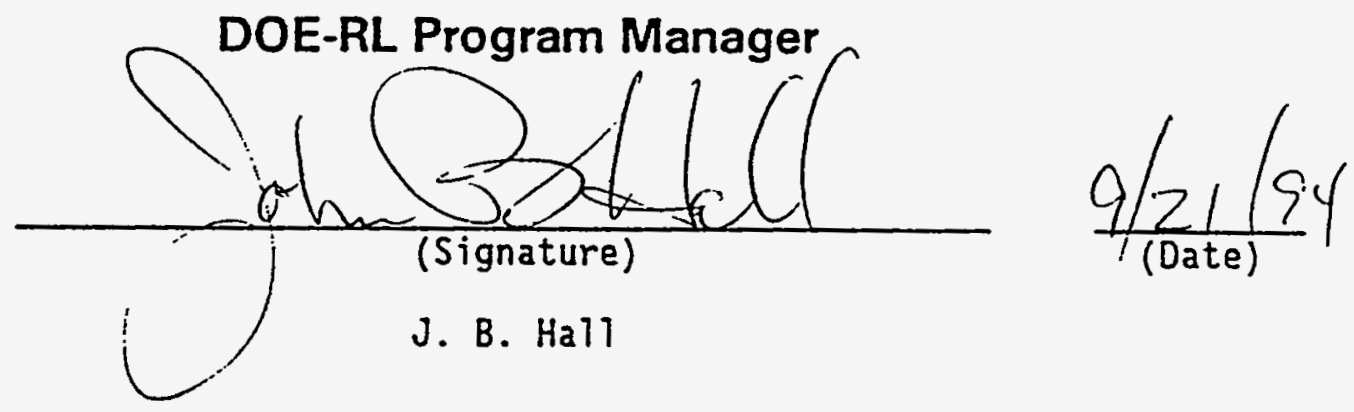

WHC Director or VP
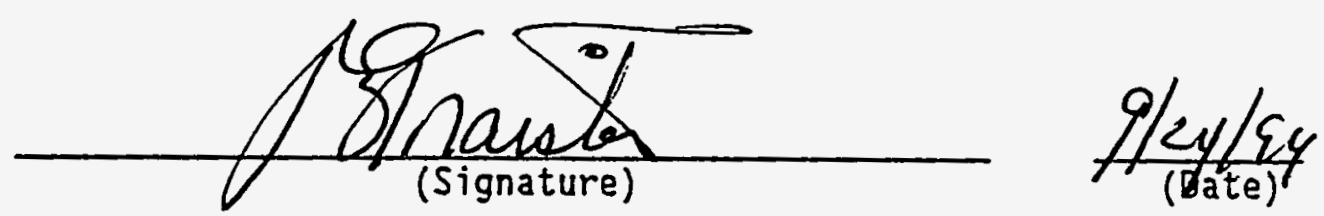

R. E. Traister

WHC Program Manager
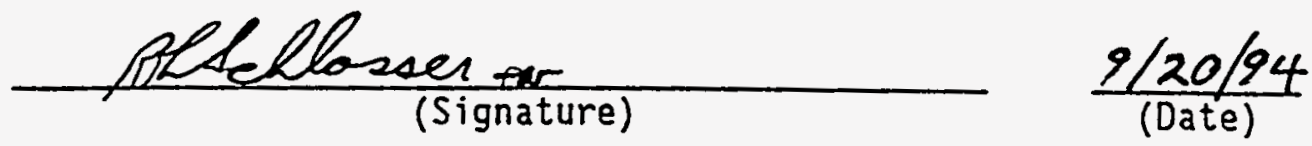

R. M. Suyama 
WHC-SP-1116 REV 0

This page intentionally left blank. 


\section{HHC-SP-1116 REV 0}

\section{CONTENTS}

1.0 Overview

1.A Vision and Mission ................. 1-1

1.B Situational Analysis .................. 1-2

1.B.1 Internal Assessment Summary ........... 1-3

1.B.2 External Assessment Summary . . . . . . . . . 1-5

1.C Goals and Objectives ....................... 1-6

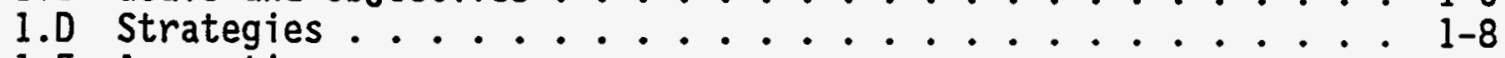

1.E Assumptions ..................... 1- . . . . . . .

1.F Issues and Constraints ............... 1-11

1.G Performance Measures . . . . . . . . . . . . . . 1-12

1.H References................. 1-13

2.0 Basetines

2.A Technical Baseline............... 2-1 2.A.1 Work Breakdown Structure and

Responsibility Assignment Matrix . . . . . . . 2-1

2.A.2 Description of Activities .......... 2-2

2.B Schedule Baseline ................. 2-3

2.B.1 Milestone List ............... 2-3

2.B.2 Program Schedule . . . . . . . . . . 2-9

2.C Cost Baseline .............. 2-15

2.C.1 Cost Basel ine by Program Element . . . . . . . 2-15

2.C.2 Cost Basis .............. 2- 2-20

2.C.3 Full-Time Equivalent Staff by Job Description .. 2-22

3.0 Execution Year Package

3.A Work Breakdown Structure Dictionary ............. 3-1

3. B Milestone Description Sheets ............. 3-5

3.C Glossary ........................ 3-27

3.D FY 1995 Task Package Contro1 Number Index ....... 3-30

3.E Cost Account PIan, B620-R Report ........... 3-31

4.0 Appendixes

4.A Appendix A................... 4-1

4.B Appendix B .................... 4- . . . . . . 


\section{WHC-SP-1116 REV 0}

\section{LIST OF TERHS}

$\begin{array}{ll}\text { CAM } & \text { corrective action management } \\ \text { CNTR } & \text { contractor } \\ \text { CSP } & \text { common support pool } \\ \text { DOE-HQ } & \text { U.S. Department of Energy, Headquarters } \\ \text { DOE-RL } & \text { U.S. Department of Energy, Richland Operations office } \\ \text { DOH } & \text { department overhead } \\ \text { EFCOG } & \text { Energy Facilities Contractors Operating Group } \\ \text { FY } & \text { fiscal year } \\ \text { G\&A } & \text { general and administrative } \\ \text { SA\&E } & \text { Safety Analysis \& Engineering } \\ \text { SMS } & \text { Site management system } \\ \text { TPCN } & \text { task package control number } \\ \text { WBS } & \text { work breakdown structure } \\ \text { WHC } & \text { Westinghouse Hanford Company }\end{array}$


WHC-SP-1116 REV 0

\begin{tabular}{|c|c|c|}
\hline 1.A Vision and Mission & $\begin{array}{c}\text { Westinghouse Hanford Company } \\
\text { Overheads, Safety Analysis \& } \\
\text { Engineering, SMS/WBS 6.3.5 }\end{array}$ & $\begin{array}{c}\text { Fite Support Program Plan } \\
\text { Date Prepared: } 9 / 14 / 94\end{array}$ \\
\hline
\end{tabular}

\section{Vision}

The Safety Analysis \& Engineering (SA\&E) department provides core competency for safety analysis and risk documentation that supports achievement of the goals and mission as described in the Hanford Mission Plan, Volume I, Site Guidance (DOE-RL 1993). SA\&E operations are integrated into the programs that plan and conduct safe waste management, environmental restoration, and operational activities. SA\&E personnel are key members of task teams assigned to eliminate urgent risks and inherent threats that exist at the Hanford Site. Key to ensuring protection of public health and safety, and that of onsite workers, are the products and services provided by the department. SA\&E will continue to provide a leadership role throughout the DOE complex with innovative, cost-effective approaches to ensuring safety during environmental cleanup operations.

\section{Mission}

The SA\&E mission is to provide support to direct program operations through safety analysis and risk documentation and to maintain an infrastructure responsive to the evolutionary climate at the Hanford Site. SA\&E will maintain the appropriate skills mix necessary to fulfill the customers' need to conduct all operations in a safe and cost-effective manner while ensuring the safety of the public and the onsite worker. 


\section{WHC-SP-1116 REV 0}

\begin{tabular}{|l|c|c|}
\hline 1.B Situational Analysis & $\begin{array}{c}\text { Westinghouse Hanford Company } \\
\text { Overheads, Safety Analysis \& } \\
\text { Engineering, SMS/WBS 6.3.5 }\end{array}$ & $\begin{array}{c}\text { FY } 1995 \\
\text { Site Support Program Plan } \\
\text { Date Prepared: } 9 / 14 / 94\end{array}$ \\
\hline
\end{tabular}

\section{Primary Customers}

The primary customers supported by this portion of the Site Support Program Plan are the SA\&E personnel; the U.S. Department of Energy, Richland Operations Office (DOE-RL); the U.S. Department of Energy, Headquarters (DOE-HQ); Westinghouse Hanford Company (WHC) direct-funded programs and projects; and operating and surplus nuclear and nonnuclear facilities. The SA\&E Department provides safety-design support and produces safetyanalysis and risk-assessment documentation, safety assessments, criticality analysis, and project safety documentation. The department is responsible for implementing DOE orders and rules for use in safety and risk analysis activities.

\section{Products and Services Provided}

This evaluation is divided into three major elements: (1) operations support, (2) program management, and

(3) qualification and training.

(1) Operations support provides the building space (occupancy), telephones, network access, computermaintenance support (through Boeing Computer Services, Richland), medical assistance (through the Hanford Environmental Health Foundation), Engineering Analys is and Technology (EAT) management proration, and building administration.

(2) Program management provides the personnel and resources to ensure implementation of the department charter, assess productivity, maintain total quality involvement within the DOE community, ensure compliance with WHC policies and procedures, trend cost effectiveness and customer satisfaction, support the Site Support Program Plan and the Environment, Health and Safety Management $\mathrm{Plan}$, and ensure that an adequate mix of required skills is available.

(3) Qualification and training for SA\&E personnel is provided. 


\begin{tabular}{|l|c|c|}
\hline 1.B.1 Internal Assessment Summary & $\begin{array}{c}\text { Westinghouse Hanford Company } \\
\text { Overheads, Safety Analysis \& } \\
\text { Engineering, SMS/WBS 6.3.5 }\end{array}$ & $\begin{array}{c}\text { FY 1995 } \\
\text { Site Support Program P1an } \\
\text { Date Prepared: 9/14/94 }\end{array}$ \\
\hline
\end{tabular}

The major strength of the organization is the ability to redirect limited resources to ever-changing priorities and to maintain the necessary skill base to do so.

\section{Major Activities Performed to Deliver Products and Services}

Goals, objectives, and values receive continuous evaluation through staff interaction, management interface with customers, and the involvement of SA\&E personnel with the Energy Facilities Contractors Operating Group (EFCOG), professional societies and standards groups, and DOE personnel complex-wide.

Department participation in the development and implementation of the Multi-Year Program Plan (direct) and the Site Support Program Plan (indirect) are major activities that determine the allocation of resources and the acquisition of skills necessary for the department to produce timely, high quality, cost-effective products.

Because safety analysis work is highly specialized, the qualification and certification program for analysts is an essential activity for ensuring high quality products. Adequate training of SA\&E personnel to ensure core competency in an ever-changing environment requires a disciplined approach to training and has a direct impact on product quality.

Contracts administration is a major activity necessary to provide the appropriate level of qualified outside services supporting direct staff in meeting commitments.

A managed program for the acquisition, deployment, and maintenance of computing hardware and software is an integral part of the department's daily operation.

As a performing organization, SA\&E is a major contributor to the policies and procedures that become part of the Nonreactor Facility Safety Analysis Manual (WHC-CM-4-46) managed by the Nuclear Safety Organization. 


\begin{tabular}{|c|c|c|}
\hline & $\begin{array}{c}\text { Westinghouse Hanford Company } \\
\text { Overheads, Safety Analysis \& } \\
\text { Engineering, SMS/WBS 6.3.5 }\end{array}$ & $\begin{array}{c}\text { FY } 1995 \\
\text { Site Support Program Plan } \\
\text { Date Prepared: } 9 / 14 / 94\end{array}$ \\
\hline
\end{tabular}

\section{Evaluation of Major Activities}

The cost of products produced could be reduced significantly by relieving the SA\&E department of a portion of the nondiscretionary costs. As an indirect program, 100\% of all occupancy costs, telephones, medicals, network connections, supplies, program developments, and mandatory training is passed on to the customers as burden. Although the dollar volume of direct-funded work has steadily increased over the last few years, the overhead-funded program has remained essentially constant. However, rising costs have reduced to a critical level the resources available to implement the program objectives. Administrative functions that inform the organization and the company of the program's effectiveness are strained. Adoption and use of sitewide management systems (e.g., Site standard scheduling system) would eliminate redundancy and reduce costs.

\section{Factors that Influence Resource Consumption}

The major factors that influence resource consumption are changes to DOE rules and orders, escalation rates, increased training requirements, changing planning and reporting requirements, reorganizations, reprioritizations of funds and personnel, fluctuations in direct-funding bases, rising material and services costs, and emergent work.

\section{Factors that Influence Changes in Types or Nature of Products and Services}

The major factors that influence changes in the types or nature of products and services are changes to planning and reporting systems and processes, rising costs, resource limitations, incomplete planning, lack of cost control, new or changing regulations, el imination of previous requirements, customer needs, and reorganizations or reassignment of organizational roles and responsibilities. 


\begin{tabular}{|l|l|l} 
1.B.2 External Assessment Summary & $\begin{array}{l}\text { Westinghouse Hanford Company } \\
\text { Overheads, Safety Analysis \& } \\
\text { Engineering, SMS/WBS 6.3.5 }\end{array}$ & $\begin{array}{c}\text { FY 1995 } \\
\text { Site Support Program Plan } \\
\text { Date Prepared: } 9 / 14 / 94\end{array}$
\end{tabular}

\section{Summary of Customer Requirements}

The customers' needs have been identified through an ongoing dialogue and will continue to evolve with time. At this point, the major expectations of SA\&E are to develop, in concert with WHC Nuclear Safety, a standardized approach to safety documentation that reflects DOE rulemaking activities, to continue a qualification and training program for analysts, and to support the Hanford Site Plan through the development of input to the Site Support Program Plan/Multi-Year Program Plan.

\section{Potential Impacts}

The strong thrust towards privatization at Hanford and the changing role of the maintenance and operations contractor to one of integrator, could precipitate some impacts to the plans developed in this program. If safety analysis and engineering capability, per se, is outsourced, the program development/excellence portion of the department's operation might be at risk for a clearly defined sponsor. The integrator would, by charter, be concerned with the programmatic aspects of the work. The service contractors would be responsible for the execution. Managed focus on these core competencies of SA\&E throughout this transition is recommended to ensure program consistency and continued progress. Additionally, it is unclear what impact increased outsourcing would have on the liquidation base that provides for this program's continued success. 


\begin{tabular}{|l|c|c|}
\hline 1.C Goals and Objectives & $\begin{array}{c}\text { Westinghouse Hanford Company } \\
\text { Overheads, Safety Analysis \& } \\
\text { Engineering, SMS/WBS 6.3.5 }\end{array}$ & $\begin{array}{c}\text { FY 1995 } \\
\text { Site Support Program Plan } \\
\text { Date Prepared: } 9 / 14 / 94\end{array}$ \\
\hline
\end{tabular}

\section{Goals}

1.0 Ensure that all SA\&E personnel work safely at all times. Provide a culture that supports safety in all facets of our employees' 1 ives.

2.0 Increase DOE customer support through improved interface meetings and better performance measurement.

3.0 Reduce the cost of support services to the direct programs.

4.0 Improve the skills mix and capabilities of the department staff in all areas of support.

5.0 Enhance processes and procedures to increase worker productivity and reduce product costs.

\section{objectives}

1.0 Maintain a high level of quality safety training and achieve $90 \%$ or better attendance at monthly safety meetings.

2.0 Ensure that all safety and risk-analysis activities are conducted in accordance with the applicable DOE rules and orders, laws, and regulations to protect the health and environment. Update, revise, and eliminate department procedures relating to quality that affect safety and risk-analysis activities.

3.0 Conduct monthly DOE customer-interface meetings to review cost, scope, and schedule for the approved work-plan milestones.

4.0 Develop resource-loaded schedules for all program milestones and review the status monthly. 


\section{WHC-SP-1116 REV 0}

\begin{tabular}{|c|c|c|}
\hline 1.C Goais and Objectives & $\begin{array}{c}\text { Westinghouse Hanford Company } \\
\text { Overheads, Safety Analys is \& } \\
\text { Engineering, SMS/WBS 6.3.5 }\end{array}$ & $\begin{array}{c}\text { FY 1995 } \\
\text { Site Support Program Plan } \\
\text { Date Prepared: } 9 / 14 / 94\end{array}$ \\
\hline
\end{tabular}

5.0 Reduce the department overhead (DOH) rate by $10 \%$ for fiscal year (FY) 1995.

6.0 Develop and implement a qualification program for safety analysts. 


\begin{tabular}{|l|c|c|}
\hline 1.D Strategies & $\begin{array}{c}\text { Westinghouse Hanford Company } \\
\text { Overheads, Safety Analysis \& } \\
\text { Engineering, SMS/WBS 6.3.5 }\end{array}$ & $\begin{array}{c}\text { FY 1995 } \\
\text { Site Support Program Plan } \\
\text { Date Prepared: } 9 / 14 / 94\end{array}$ \\
\hline
\end{tabular}

\section{Customer Support Strategies}

The major program elements have been determined through continuous customer interface. Those qualityaffecting elements receive input from internal-staff management and from our DOE customer. The SA\&E program must be responsive to changes in DOE guidance/regulations as well as the needs of our internal customers. Constant communication via biweekly interface meetings with the DOE-RL monitor ensure consensus direction of ongoing activities.

SA\&E products and services provided to the direct-funded programs are identified in each program's input to the Multi-Year Program Plan and the Fiscal Year Program Plans. Additionally, direct funding is often provided through cross charging of task package control numbers (TPCNs) and work orders for activities related to urgent risks and emergent work.

\section{Production Strategies}

Products are first prioritized in order of importance (need). Cost estimates for each deliverable, and an accompanying time frame, are determined. Finally, the availability of resources, personnel, and money are considered to determine how much can be accomplished in a given fiscal year. Milestone commitments are clearly defined, and the dedicated resources necessary to accomplish them are identified. A major factor in success is to have deliverables described in enough detail that all involved understand what is expected. All milestone deliverables are accompanied by resource-loaded schedules that are reviewed monthiy. 


\begin{tabular}{|l|c|c|}
\hline 1.D Strategies & $\begin{array}{c}\text { Westinghouse Hanford Company } \\
\text { Overheads, Safety Analys is \& } \\
\text { Engineering, SMS/WBS 6.3.5 }\end{array}$ & $\begin{array}{c}\text { Fy 1995 } \\
\text { Site Support Program Plan } \\
\text { Date Prepared: } 9 / 14 / 94\end{array}$ \\
\hline
\end{tabular}

\section{Organization and Management Strategies}

The department is essentially structured to support our major, WHC project customers. However, because the products of the support program often benefit all of our customers, organizational structures need to be flexible enough to support changing needs. A management system responsive to changing priorities is essential in today's environment. The department manager has an ongoing evaluation of organizational responsiveness to customer needs and the skills mix of personnel within the department. 
WHC-SP-1116 REV 0

\begin{tabular}{|l|c|c|}
\hline 1.E Assumptions & $\begin{array}{c}\text { Westinghouse Hanford Company } \\
\text { Overheads, Safety Analysis \& } \\
\text { Engineering, SMS/WBS 6.3.5 }\end{array}$ & $\begin{array}{c}\text { FY 1995 } \\
\text { Site Support Program Plan } \\
\text { Date Prepared: } 9 / 14 / 94\end{array}$ \\
\hline
\end{tabular}

The following assumptions are the basis for developing the goals and objectives of this plan for FY 1995 .

Assumption 1: Direct funding to support the liquidation labor base for this program will be available at the level necessary. At this time, the direct funding is based on best estimates developed with the program office representatives.

Assumption 2: The SA\&E department will remain intact and be able to adjust the skills mix of the personnel to more effectively support our customers.

Assumption 3: Creative ways will be developed to have the programs bear the nondiscretionary costs associated with hiring contract personnel to support the workload at hand.

Assumption 4: The program management infrastructure currently in place will continue to function and to provide increased visibility to. our DOE customers on program performance. 


\section{WHC-SP-1116 REV 0}

\begin{tabular}{|l|c|c|}
\hline 1.F Issues and Constraints & $\begin{array}{c}\text { Westinghouse Hanford Company } \\
\text { Overheads, Safety Analys is \& } \\
\text { Engineering, SMS/WBS 6.3.5 }\end{array}$ & $\begin{array}{c}\text { FY 1995 } \\
\text { Site Support Program P1 an } \\
\text { Date Prepared: } 9 / 14 / 94\end{array}$ \\
\hline
\end{tabular}

Significant increases in outsourcing of work could impact this program. This activity does not bear burden for department overhead and would significantly reduce funds available for the Safety Analysis \& Engineering program. However, work scope could be maintained with a significant rate increase. If funds are reduced, the discretionary portion of the program would have reduced work scope in order to meet nondiscretionary expenses.

No known obstacles exist, at present, to prevent accomplishment of the program's objectives. 


\begin{tabular}{|l|c|c|}
\hline 1.G Performance Measures & $\begin{array}{c}\text { Westinghouse Hanford Company } \\
\text { Overheads, Safety Analys is \& } \\
\text { Engineering, SMS/WBS 6.3.5 }\end{array}$ & $\begin{array}{c}\text { FY } 1995 \\
\text { Site Support Program Plan } \\
\text { Date Prepared: 9/14/94 }\end{array}$ \\
\hline
\end{tabular}

The SA\&E organization maintains a total quality plan that utilizes performance indicators to assess performance and to $\mathrm{pl}$ an and indicate the need for corrective actions and improvements. The performance measures are provided on a monthly basis to management.

1.0 Meet a least $90 \%$ of commitment dates.

2.0 Provide analyses and corrective action plans for all variances exceeding a threshold of plus or minus $10 \%$ and $\$ 25,000$. Monitor corrective action plans and evaluate them for success.

3.0 Meet at least $95 \%$ of the QUEST response dates.

4.0 Submit ECCEL requests that meet or exceed the ECCEL dollar target for FY 1995.

5.0 Achieve $90 \%$ or better attendance at monthly safety meetings. 
WHC-SP-1116 REV 0

\begin{tabular}{|l|c|c|}
\hline $1 . H \quad R e f e r e n c e s$ & $\begin{array}{c}\text { Westinghouse Hanford Company } \\
\text { Overheads, Safety Analysis \& } \\
\text { Engineering, SMS/WBS 6.3.5 }\end{array}$ & $\begin{array}{c}\text { FY 1995 } \\
\text { Site Support Program Plan } \\
\text { Date Prepared: 9/14/94 }\end{array}$ \\
\hline
\end{tabular}

DOE-RL, 1993, Hanford Mission Plan, Volume I, Site Guidance, D0E-RL-93-0008, U.S. Department of Energy, Richland Operations Office, Richland, Washington.

WHC-CM-4-46, Nonreactor Facility Safety Analysis Manual, Westinghouse Hanford Company, Richland, Washington. 


\section{WHC-SP-1116 REV 0}

This page intentionally left blank.

Overheads, Safety Analysis \& Engineering Page 1-14 


\section{WHC-SP-1116 REV 0}

\begin{tabular}{|c|c|c|c|c|c|}
\hline \multirow{2}{*}{\multicolumn{2}{|c|}{$\begin{array}{l}\text { 2.A Technical Baseline } \\
\text { 2.A.1 Work Breakdown } \\
\text { Structure and } \\
\text { Responsibility Assignment } \\
\text { Matrix }\end{array}$}} & \multirow{2}{*}{\multicolumn{2}{|c|}{$\begin{array}{l}\text { Westinghouse Hanford Company } \\
\text { Overheads, Safety Analysis \& } \\
\text { Engineering, SMS/WBS } 6.3 .5\end{array}$}} & \multirow{2}{*}{\multicolumn{2}{|c|}{$\begin{array}{c}\text { FY } 1995 \\
\text { Site Support Program Plan } \\
\text { Date Prepared: } 9 / 14 / 94\end{array}$}} \\
\hline & & & & & \\
\hline DOE-RL WBS & $\begin{array}{l}\text { Activity } \\
\text { (See 2.A.2) }\end{array}$ & $\begin{array}{c}\text { Cost } \\
\text { account }\end{array}$ & $\begin{array}{l}\text { Title (DOE-RL WBS, } \\
\text { activity, cost } \\
\text { account) }\end{array}$ & $\begin{array}{l}\text { Responsible } \\
\text { manager }\end{array}$ & $\begin{array}{c}\text { Responsible } \\
\text { organization } \\
\text { (organization code) }\end{array}$ \\
\hline \multirow[t]{5}{*}{6.3} & 6.3 .5 & $1 \mathrm{~J} 8010$ & $\begin{array}{l}\text { Overheads, Safety } \\
\text { Analysis \& } \\
\text { Engineering }\end{array}$ & E. P. DiVincenzo & $\begin{array}{l}\text { Safety Engineering } \\
\text { Programs (8D170) }\end{array}$ \\
\hline & 6.3 .5 .1 & & $\begin{array}{l}\text { Management and } \\
\text { Administration. }\end{array}$ & E. P. DiVincenzo & $\begin{array}{l}\text { Safety Engineering } \\
\text { Programs }(80170)\end{array}$ \\
\hline & 6.3 .5 .2 & & Program Management & E. P. DiVincenzo & $\begin{array}{l}\text { Safety Engineering } \\
\text { Programs }(80170)\end{array}$ \\
\hline & 6.3 .5 .3 & & $\begin{array}{l}\text { Qualifications and } \\
\text { Training }\end{array}$ & E. P. DiVincenzo & $\begin{array}{l}\text { Safety Engineering } \\
\text { Programs }(80170)\end{array}$ \\
\hline & 6.3 .5 .4 & & Emergent Work & E. P. DiVincenzo & $\begin{array}{l}\text { Safety Engineering } \\
\text { Programs (8D170) }\end{array}$ \\
\hline
\end{tabular}




\begin{tabular}{|c|c|c|c|}
\hline $\begin{array}{l}\text { 2.A.2 Description } \\
\text { of Activities }\end{array}$ & $\begin{array}{l}\text { West } i \\
\text { Overheads, }\end{array}$ & $\begin{array}{l}\text { Ise Hanford Company } \\
\text { ty Analysis \& Engineering } \\
\text { S/WBS } 6.3 .5\end{array}$ & $\begin{array}{l}\text { FY } 1995 \\
\text { Site Support Program Plan } \\
\text { Date Prepared: } 9 / 14 / 94\end{array}$ \\
\hline Activity number & Activity title & \multicolumn{2}{|c|}{ Description } \\
\hline 6.3 .5 .1 & $\begin{array}{l}\text { Management and } \\
\text { Administration }\end{array}$ & \multicolumn{2}{|c|}{$\begin{array}{l}\text { Management and Administration encompasses the management and } \\
\text { administrative activities associated with the operations of } \\
\text { the SA\&E department and the management of department } \\
\text { resources. This includes management of fixed departmental } \\
\text { costs, discretionary functional management and } \\
\text { administration activities, provision for hourly support } \\
\text { personnel, and resources for departmental staff moves. }\end{array}$} \\
\hline 6.3 .5 .2 & Program Management & \multicolumn{2}{|c|}{$\begin{array}{l}\text { Program Management addresses budgetary and planning input to } \\
\text { the Multi-Year Program Plan, Fiscal Year Work Plan, and Site } \\
\text { Support Program Plan. } \\
\text { Contract Administration supports the SA\&E organization's } \\
\text { role in the administration and procurement of subcontracted } \\
\text { services (safety analysis documents). } \\
\text { Also included is the performance of monthly program reviews, } \\
\text { monthly DOE-interface meetings, development of performance } \\
\text { analysis/indicators, productivity evaluations, process } \\
\text { change control actions, input to the monthly Site Management } \\
\text { System Report, and management of the automated data- } \\
\text { processing equipment for the department. } \\
\text { This activity also manages EFCOG involvement, professional } \\
\text { societies requirements, and corrective action activities } \\
\text { for the SA\&E department. }\end{array}$} \\
\hline $6.3 \cdot 5.3$ & $\begin{array}{l}\text { Qualification and } \\
\text { Certification } \\
\text { Training }\end{array}$ & \multicolumn{2}{|c|}{$\begin{array}{l}\text { Qualification and Certification Training develops training } \\
\text { requirements criteria and training plans and trains } \\
\text { analysts. }\end{array}$} \\
\hline 6.3 .5 .4 & Emergent Work & \multicolumn{2}{|c|}{$\begin{array}{l}\text { Emergent Work manages imposed, unbudgeted, and unplanned } \\
\text { work scope. }\end{array}$} \\
\hline
\end{tabular}




\section{WHC-SP-1116 REV 0}

\begin{tabular}{|c|c|c|c|c|}
\hline \multicolumn{3}{|c|}{ 2.B Schedule Baseline } & \multirow{2}{*}{$\begin{array}{l}\text { Westinghouse Hanford Company } \\
\text { Overheads, Safety Analysis \& } \\
\text { Engineering, SMS/WBS } 6.3 .5\end{array}$} & \multirow{2}{*}{$\begin{array}{c}\text { FY } 1995 \\
\text { Site Support Program Plan } \\
\text { Date Prepared: } \quad 9 / 14 / 94 \\
\end{array}$} \\
\hline \multirow{2}{*}{$\begin{array}{l}\text { 2.B.1 } \\
\text { Type } \\
\end{array}$} & \multicolumn{2}{|c|}{ Milestone List } & & \\
\hline & Number & WBS Number & Milestone description & Due date \\
\hline \multicolumn{5}{|c|}{$\therefore \quad: \quad:$} \\
\hline CNTR & SAE-95-001 & 6.3 .5 .2 .1 & $\begin{array}{l}\text { Safety Analysis \& Engineering FY } 1995 \text { Department Overhead } \\
\text { (DOH) Mid-Year Review }\end{array}$ & $4 / 28 / 95$ \\
\hline CNTR & SAE-95-002 & 6.3 .5 .2 .1 & Safety Analysis \& Engineering FY 1996 DOH Budget & $8 / 31 / 95$ \\
\hline CNTR & SAE-95-003 & 6.3 .5 .2 .1 & $\begin{array}{l}\text { Draft of Safety Analysis \& Engineering FY } 1996 \text { Site Support } \\
\text { Program Plan/Multi-Year Program Plan }\end{array}$ & $6 / 30 / 95$ \\
\hline DOE-RL & SAE-95-004 & 6.3 .5 .2 .1 & $\begin{array}{l}\text { Safety Analysis \& Engineering FY } 1996 \text { Site Support Program } \\
\text { Plan/Multi-Year Program Plan }\end{array}$ & $8 / 31 / 95$ \\
\hline CNTR & SAE-95-005 & 6.3 .5 .2 .1 & $\begin{array}{l}\text { Automated Data-Processing Equipment Mid-Year Statement of } \\
\text { Strategy for FY } 1995\end{array}$ & $4 / 14 / 95$ \\
\hline CNTR & SAE-95-006 & 6.3 .5 .2 .1 & $\begin{array}{l}\text { Automated Data-Processing Equipment Procurement Plan for } \\
\text { FY } 1996\end{array}$ & $7 / 28 / 95$ \\
\hline CNTR & SAE-95-007 & 6.3 .5 .2 .1 & Safety Analysis \& Engineering Monthly Performance Statistics & $9 / 15 / 95$ \\
\hline CNTR & SAE-95-008 & 6.3 .5 .2 .1 & Safety Analysis \& Engineering Site Management System Report & $9 / 15 / 95$ \\
\hline CNTR & SAE-95-009 & 6.3 .5 .2 .1 & $\begin{array}{l}\text { Safety Analysis \& Engineering Variance and Corrective Action } \\
\text { Report }\end{array}$ & $9 / 15 / 95$ \\
\hline CNTR & SAE-95-010 & 6.3 .5 .2 .1 & $\begin{array}{l}\text { Safety Analysis \& Engineering 1J8D10 Program Development and } \\
\text { Implementation Program Review }\end{array}$ & $9 / 29 / 95$ \\
\hline
\end{tabular}


WHC-SP-1116 REV 0

\begin{tabular}{|c|c|c|c|c|}
\hline \multicolumn{3}{|c|}{ 2.B Schedule Baseline } & \multirow{2}{*}{$\begin{array}{c}\text { Westinghouse Hanford Company } \\
\text { Overheads, Safety Analys is \& } \\
\text { Engineering, SMS/WBS } 6.3 .5\end{array}$} & \multirow{2}{*}{$\begin{array}{c}\text { FY } 1995 \\
\text { Site Support Program Plan } \\
\text { Date Prepared: } 9 / 14 / 94 \\
\end{array}$} \\
\hline \multirow{2}{*}{$\frac{2 . B .1}{\text { Type }}$} & \multicolumn{2}{|c|}{ Milestone List } & & \\
\hline & Number & WBS Number & Milestone description & Due date \\
\hline$D O E-R L$ & SAE-95-011 & 6.3 .5 .2 .1 & $\begin{array}{l}\text { Quarterly Progress Report of Safety Analysis \& Engineering } \\
\text { Program }\end{array}$ & $1 / 16 / 95$ \\
\hline DOE-RL & SAE-95-012 & 6.3 .5 .2 .1 & $\begin{array}{l}\text { Quarterly Progress Report of Safety Analysis \& Engineering } \\
\text { Program }\end{array}$ & $4 / 14 / 95$ \\
\hline DOE-RL & SAE-95-013 & 6.3 .5 .2 .1 & $\begin{array}{l}\text { Quarterly Progress Report of Safety Analysis \& Engineering } \\
\text { Program }\end{array}$ & $7 / 14 / 95$ \\
\hline DOE-RL & SAE-95-014 & 6.3 .5 .2 .1 & $\begin{array}{l}\text { Quarteriy Progress Report of Safety Analysis \& Engineering } \\
\text { Program }\end{array}$ & $9 / 29 / 95$ \\
\hline CNTR & SAE-95-015 & 6.3 .5 .3 .1 & $\begin{array}{l}\text { Safety Analysis \& Engineering Annual Update of Training } \\
\text { Records }\end{array}$ & $8 / 31 / 95$ \\
\hline CNTR & SAE-95-016 & 6.3 .5 .2 .4 & Monthiy Status Report of Subcontractor Support & $9 / 29 / 95$ \\
\hline DOE-RL & SAE-95-017 & 6.3 .5 .2 .4 & Quarterly Outsourcing Status Report & $1 / 16 / 95$ \\
\hline DOE-RL & SAE-95-018 & 6.3 .5 .2 .4 & Quarterly Outsourcing Status Report & $4 / 14 / 95$ \\
\hline DOE-RL & SAE-95-019 & 6.3 .5 .2 .4 & Quarterly Outsourcing Status Report & $7 / 14 / 95$ \\
\hline DOE-RL & SAE-95-020 & 6.3 .5 .2 .4 & Quarterly Outsourcing Status Report & $9 / 29 / 95$ \\
\hline CNTR & SAE-95-021 & 6.3 .5 .2 .1 & $\begin{array}{l}\text { Draft Outline of Safety Analysis \& Engineering Transition } \\
\text { Plan }\end{array}$ & $11 / 15 / 95$ \\
\hline DOE-RL & SAE-95-022 & 6.3 .5 .2 .1 & Safety Analysis \& Engineering Transition Plan & $1 / 15 / 95$ \\
\hline \multicolumn{5}{|c|}{ Fiscal Year 1996} \\
\hline CNTR & SAE-96-001 & 6.3 .5 .2 .1 & Safety Analysis \& Engineering FY 1996 DOH Mid-Year Review & $4 / 30 / 96$ \\
\hline
\end{tabular}

Overheads, Safety Analysis \& Engineering Page 2-4 
WHC-SP-1116 REV 0

\begin{tabular}{|c|c|c|c|c|}
\hline \multicolumn{3}{|c|}{ 2.B Schedule Baseline } & \multirow{2}{*}{$\begin{array}{c}\text { Westinghouse Hanford Company } \\
\text { Overheads, Safety Analysis \& } \\
\text { Engineering, SMS/WBS } 6.3 .5 \\
\end{array}$} & \multirow{2}{*}{$\begin{array}{l}\text { FY } 1995 \\
\text { Site Support Program Plan } \\
\text { Date Prepared: } 9 / 14 / 94 \\
\end{array}$} \\
\hline \multirow{2}{*}{$\frac{2 . B .1}{\text { Type }}$} & \multicolumn{2}{|c|}{ Milestone List } & & \\
\hline & Number & WBS Number & Milestone description & Due date \\
\hline CNTR & SAE-96-002 & 6.3 .5 .2 .1 & Safety Analysis \& Engineering FY 1997 DOH Budget & $8 / 30 / 96$ \\
\hline CNTR & SAE-96-003 & 6.3 .5 .2 .1 & $\begin{array}{l}\text { Draft of Safety Analysis \& Engineering FY } 1997 \text { Site Support } \\
\text { Program Plan/Multi-Year Program Plan }\end{array}$ & $6 / 28 / 96$ \\
\hline DOE-RL & SAE-96-004 & 6.3 .5 .2 .1 & $\begin{array}{l}\text { Safety Analysis \& Engineering FY } 1997 \text { Site Support Program } \\
\text { Plan/Multi-Year Program Plan }\end{array}$ & $8 / 30 / 96$ \\
\hline CNTR & SAE-96-005 & 6.3 .5 .2 .1 & $\begin{array}{l}\text { Automated Data-Processing Equipment Mid-Year Statement of } \\
\text { Strategy for FY } 1996\end{array}$ & $4 / 15 / 96$ \\
\hline CNTR & SAE-96-006 & 6.3 .5 .2 .1 & $\begin{array}{l}\text { Automated Data-Processing Equipment Procurement Plan for } \\
\text { FY } 1997\end{array}$ & $7 / 29 / 96$ \\
\hline CNTR & SAE-96-007 & 6.3 .5 .2 .1 & Safety Analysis \& Engineering Monthly Performance Statistics & $9 / 16 / 96$ \\
\hline CNTR & SAE-96-008 & 6.3 .5 .2 .1 & Safety Analysis \& Engineering Site Management System Report & $9 / 16 / 96$ \\
\hline CNTR & SAE-96-009 & 6.3 .5 .2 .1 & $\begin{array}{l}\text { Safety Analysis \& Engineering Variance and Corrective Action } \\
\text { Report }\end{array}$ & $9 / 16 / 96$ \\
\hline CNTR & SAE-96-010 & $6.3 \cdot 5.2 .1$ & $\begin{array}{l}\text { Safety Analysis \& Engineering } 1 \text { J8D10 Program Development and } \\
\text { Implementation Program Review }\end{array}$ & $9 / 30 / 96$ \\
\hline DOE-RL & SAE-96-011 & 6.3 .5 .2 .1 & $\begin{array}{l}\text { Quarterly Progress Report of Safety Analysis \& Engineering } \\
\text { Program }\end{array}$ & $1 / 15 / 96$ \\
\hline DOE-RL & SAE-96-012 & 6.3 .5 .2 .1 & $\begin{array}{l}\text { Quarterly Progress Report of Safety Analysis \& Engineering } \\
\text { Program }\end{array}$ & $4 / 15 / 96$ \\
\hline DOE-RL & SAE-96-013 & $6.3 \cdot 5.2 .1$ & $\begin{array}{l}\text { Quarterly Progress Report of Safety Analysis \& Engineering } \\
\text { Program }\end{array}$ & $7 / 15 / 96$ \\
\hline
\end{tabular}

Overheads, Safety Analysis \& Engineering Page 2-5 
WHC-SP-1116 REV 0

\begin{tabular}{|c|c|c|c|c|}
\hline \multicolumn{3}{|c|}{ 2.B Schedule Baseline } & \multirow{2}{*}{$\begin{array}{c}\text { Westinghouse Hanford Company } \\
\text { Overheads, Safety Analysis \& } \\
\text { Engineering, SMS/WBS } 6.3 .5\end{array}$} & \multirow{2}{*}{$\begin{array}{l}\text { Fy } 1995 \\
\text { Site Support Program Plan } \\
\text { Date Prepared: } 9 / 14 / 94 \\
\end{array}$} \\
\hline \multirow{2}{*}{ 2.B.1 } & \multicolumn{2}{|c|}{ Milestone List } & & \\
\hline & Number & WBS Number & Milestone description & Due date \\
\hline DOE-RL & SAE-96-014 & 6.3 .5 .2 .1 & $\begin{array}{l}\text { Quarterly Progress Report of Safety Analysis \& Engineering } \\
\text { Program }\end{array}$ & $9 / 30 / 96$ \\
\hline CNTR & SAE-96-015 & $6.3 \cdot 5 \cdot 3.1$ & $\begin{array}{l}\text { Safety Analysis \& Engineering Annual Update of Training } \\
\text { Records }\end{array}$ & $8 / 30 / 96$ \\
\hline CNTR & SAE-96-016 & 6.3 .5 .2 .4 & Monthly Status Report of Subcontractor Support & $9 / 30 / 96$ \\
\hline DOE-RL & SAE-96-017 & 6.3 .5 .2 .4 & Quarterly Outsourcing Status Report & $1 / 15 / 96$ \\
\hline DOE-RL & SAE-96-018 & 6.3 .5 .2 .4 & Quarterly Outsourcing Status Report & $4 / 15 / 96$ \\
\hline DOE-RL & SAE-96-019 & 6.3 .5 .2 .4 & Quarterly Outsourcing Status Report & $7 / 15 / 96$ \\
\hline DOE-RL & SAE-96-020 & 6.3 .5 .2 .4 & Quarterly Outsourcing Status Report & $9 / 30 / 96$ \\
\hline \multicolumn{5}{|c|}{$\therefore$} \\
\hline CNTR & SAE-97-001 & 6.3 .5 .2 .1 & Safety Analysis \& Engineering FY 1997 DOH Mid-Year Review & $4 / 30 / 97$ \\
\hline CNTR & SAE-97-002 & 6.3 .5 .2 .1 & Safety Analysis \& Engineering FY $1998 \mathrm{DOH}$ Budget & $8 / 29 / 97$ \\
\hline CNTR & SAE-97-003 & 6.3 .5 .2 .1 & $\begin{array}{l}\text { Draft of Safety Analysis \& Engineering FY } 1998 \text { Site Support } \\
\text { Program Plan/Multi-Year Program Plan }\end{array}$ & $6 / 30 / 97$ \\
\hline DOE-RL & SAE-97-004 & 6.3 .5 .2 .1 & $\begin{array}{l}\text { Safety Analysis \& Engineering FY } 1998 \text { Site Support Program } \\
\text { Plan/Multi-Year Program Plan }\end{array}$ & $8 / 29 / 97$ \\
\hline CNTR & SAE-97-005 & 6.3 .5 .2 .1 & $\begin{array}{l}\text { Automated Data-Processing Equipment Mid-Year Statement of } \\
\text { Strategy for FY } 1997\end{array}$ & $4 / 15 / 97$ \\
\hline CNTR & SAE-97-006 & 6.3 .5 .2 .1 & $\begin{array}{l}\text { Automated Data-Processing Equipment Procurement Plan for } \\
\text { FY } 1998\end{array}$ & $7 / 28 / 97$ \\
\hline
\end{tabular}

Overheads, Safety Analysis \& Engineering Page 2-6 
WHC-SP-1116 REV 0

\begin{tabular}{|c|c|c|c|c|}
\hline \multicolumn{3}{|c|}{ 2.B Schedule Baseline } & \multirow{2}{*}{$\begin{array}{c}\text { Westinghouse Hanford Company } \\
\text { Overheads, Safety Analysis \& } \\
\text { Engineering, SMS/WBS } 6.3 .5\end{array}$} & \multirow{2}{*}{$\begin{array}{l}\text { FY } 1995 \\
\text { Site Support Program Plan } \\
\text { Date Prepared: } 9 / 14 / 94 \\
\end{array}$} \\
\hline \multirow{2}{*}{$\begin{array}{l}\text { 2.B. } 1 \\
\text { Type }\end{array}$} & \multicolumn{2}{|c|}{ Milestone List } & & \\
\hline & Number & WBS Number & Milestone description & Due date \\
\hline CNTR & SAE-97-007 & 6.3 .5 .2 .1 & Safety Analysis \& Engineering Monthly Performance Statistics & $9 / 15 / 97$ \\
\hline CNTR & SAE-97-008 & 6.3 .5 .2 .1 & Safety Analysis \& Engineering Site Management System Report & $9 / 15 / 97$ \\
\hline CNTR & SAE-97-009 & 6.3 .5 .2 .1 & $\begin{array}{l}\text { Safety Analysis \& Engineering Variance and Corrective Action } \\
\text { Report }\end{array}$ & $9 / 15 / 97$ \\
\hline CNTR & $S A E-97-010$ & 6.3 .5 .2 .1 & $\begin{array}{l}\text { Safety Analysis \& Engineering } 1 \mathrm{~J} 8010 \text { Program Development and } \\
\text { Implementation Program Review }\end{array}$ & $9 / 30 / 97$ \\
\hline DOE-RL & $S A E-97-011$ & 6.3 .5 .2 .1 & $\begin{array}{l}\text { Quarterly Progress Report of Safety Analysis \& Engineering } \\
\text { Program }\end{array}$ & $1 / 15 / 97$ \\
\hline DOE-RL & SAE-97-012 & 6.3 .5 .2 .1 & $\begin{array}{l}\text { Quarterly Progress Report of Safety Analysis \& Engineering } \\
\text { Program }\end{array}$ & $4 / 15 / 97$ \\
\hline DOE-RL & SAE-97-013 & 6.3 .5 .2 .1 & $\begin{array}{l}\text { Quarterly Progress Report of Safety Analysis \& Engineering } \\
\text { Program }\end{array}$ & $7 / 15 / 97$ \\
\hline DOE-RL & SAE-97-014 & 6.3 .5 .2 .1 & $\begin{array}{l}\text { Quarterly Progress Report of Safety Analysis \& Engineering } \\
\text { Program }\end{array}$ & $9 / 30 / 97$ \\
\hline CNTR & SAE-97-015 & $6.3 \cdot 5 \cdot 3.1$ & $\begin{array}{l}\text { Safety Analysis \& Engineering Annual Update of Training } \\
\text { Records }\end{array}$ & $8 / 29 / 97$ \\
\hline CNTR & SAE-97-016 & 6.3 .5 .2 .4 & Monthly Status Report of Subcontractor Support & $9 / 30 / 97$ \\
\hline DOE-RL & SAE-97-017 & 6.3 .5 .2 .4 & Quarterly Outsourcing Status Report & $1 / 15 / 97$ \\
\hline DOE-RL & SAE-97-018 & 6.3 .5 .2 .4 & Quarterly Outsourcing Status Report & $4 / 15 / 97$ \\
\hline DOE-RL & SAE-97-019 & 6.3 .5 .2 .4 & Quarterly Outsourcing Status Report & $7 / 15 / 97$ \\
\hline DOE-RL & SAE-97-020 & 6.3 .5 .2 .4 & Quarterly Outsourcing Status Report & $9 / 30 / 97$ \\
\hline
\end{tabular}

Overheads, Safety Analysis \& Engineering Page 2-7 
WHC-SP-1116 REV 0

This page intentionally left blank.

Overheads, Safety Analysis \& Engineering Page 2-8 
SCHEDULER JIM JUSTIN

\begin{tabular}{|c|c|c|c|}
\hline $\begin{array}{l}\text { ACT } \\
\text { I. D. }\end{array}$ & $\begin{array}{c}\text { ACT } \\
\text { DESCRIPTION }\end{array}$ & $\begin{array}{l}\text { MILESTON } \\
\text { TYPE }\end{array}$ & $\begin{array}{c}\text { MILESTONE } \\
\text { NUMBER }\end{array}$ \\
\hline $1 \mathrm{~J} 80100 \mathrm{B0} 00 \mathrm{~A}$ & PREPARE SA\&E FY 1995 OOH MID YEAR REVIEW & & \\
\hline $1 \mathrm{~J} 801008000 \mathrm{AA}$ & SA\&E FY 1995 DDH MID YEAR REVIEW & WHC-KEY & SAE-95-00 \\
\hline 1 I I801008000B. & PREPARE SA\&E FY 1996 DOH BUDGET & & \\
\hline $1 \mathrm{~J} 8010 \mathrm{CBOOOBB}$ & SA\&E FY 1996 DOH BUDGET & WHC-KEY & SAE-95-00' \\
\hline 1.J80100B000C. & PREFARE DRAFT SA\&E FY 1996 SSPP/MYPF & & \\
\hline $1 \mathrm{~J} 8 \mathrm{C} 100 \mathrm{~B} 000 \mathrm{CC}$ & DRAFT SHRE FY 1996 SSPP MYYPP & WHC-KEY & SAE-95-00: \\
\hline $1 \mathrm{s010080000.}$ & FREPARE SA\&E FY 1996 SSPP/MYPP & & \\
\hline 1.180100800000 & SAEE FY 1996 SSPP/MYFP & RL & SAE $-95-00<$ \\
\hline 1 J8[I1 OCBOOOE. & DEV ADP EOUIP MIO-YEAR STATE OF STRATEGY FY9S & & \\
\hline $1 \mathrm{JBO} 10 \mathrm{CBOOOEE}$ & ADP EQUIP MID-YEAR STATE OF STRATEGY FOR FY 1995 & WHC-KEY & SAE-95-005 \\
\hline $1.18010 \mathrm{OBO} 000 \mathrm{~F}$. & DEV AOP EQUIP FROCUREMENT PLAN FOR FY 1996 & & \\
\hline $1 \mathrm{~J} 8[1100 \mathrm{BOOOOFF}$ & FIDF EDUIP PROCUREMENT PLAN FOR FY 1996 & WHC-KEY & SAE-95-00E \\
\hline 1 I80100B000G. & PREF QUARTEFI Y PROGRESS REPORT OF SA\&E PROGRAM & & \\
\hline 1 J8[1100BOOOGG & GUARTERLY PROGFESS REPORT OF SA\&E PROGRAM & RL & SAE-95-011 \\
\hline $1 \mathrm{~J} 80100 \mathrm{~B} 000 \mathrm{H}$ & FREP OUAFTERLY PROGRESS REFORT OF SA\&E PROGRAM & & \\
\hline $1.180100 \mathrm{BOOOOHH}$ & DUARTERLY FFOGRESS FEPORT OF SA\&E PROGRAM & RL & SAE-95-01c \\
\hline 1 J8[110@BO00 1 . & PREP OUARTERLY PROGRESS REPORT OF SA\&E PROGRAM & & \\
\hline $1 \mathrm{~J} 80100 \mathrm{~B} 000 \mathrm{JJ}$ & QUARTERLY PROGRESS REPORT OF SA\&E FROGRAM & $R L$ & $S A E-95-01:$ \\
\hline 1 I I80IOS.BOOOK. & FREP QUARTERLY Y PROGRESS REPORT OF SA\&E PROGRAM & & \\
\hline $1 \mathrm{~J} 801008000 \mathrm{KK}$ & QUARTERLY PROGRESS REPORT OF SA\&E PROGRAM & RL & SAE-95-01 \\
\hline $1.180100 \mathrm{BO} 00 \mathrm{~L}$. & PREFRFE SAZE MONTHLY PERFORMANCE STATISTICS & & \\
\hline $1 \mathrm{~J} 801100 \mathrm{~B} 00 \mathrm{OLL}$ & SA\&E MONTHLY PERFORMANCE STATISTICS & WHC-KEY & SAE-95-00\% \\
\hline $1 \mathrm{~J} B \mathrm{D} 100 \mathrm{BO} 00 \mathrm{M}$. & FREPARE SA\&E SITE MANAGEMENT SYSTEM REPORT & & \\
\hline $1.180100 B 000 \mathrm{MM}$ & SA\&E SITE MANAGEMENT SYSTEM REPORT & WHC-KEY & SAE-95-00E \\
\hline $1 \mathrm{~J} 8[1100 \mathrm{BO} 00 \mathrm{~N}$ & FREP SA\&E VARIANCE AND CORRECTIVE ACTION REPORT & & \\
\hline $1.180100 \mathrm{BO} 00 \mathrm{NN}$ & SARE VARIANCE AND CORRECTIVE ACTION REPORT & WHC-KEY & SAE $-95-005$ \\
\hline 1 J $1801008000 P$. & STAPIT SA\&E 1 J8010 PROG DEV AND IMPLEM PROG REV & WHC-KEY & \\
\hline $1 \mathrm{~J} 8011008000 \mathrm{PP}$ & SA\&E 1 J8010 PROG DEV AND IMPLEM PROG REV & WHC-KEY & SAE-95-01 \\
\hline $1.1801008001 \mathrm{G}$. & PREPARE ORAFT OUTLINE SA\&E TRANSITION PLAN & & \\
\hline $1 \mathrm{~J} 801008001 \mathrm{GG}$ & DRAFT OUTLINE SA\&E TRANSITION PLAN & WHC-KEY & SAE $-95-0$ \\
\hline $1 \mathrm{JBC} 1008001 \mathrm{H}$ & FREPARE TRANSITION PLAN & & \\
\hline
\end{tabular}




\section{IE HANFORD CO.}

PPORT PROGRAM SCHEDULE

$S P-1116$

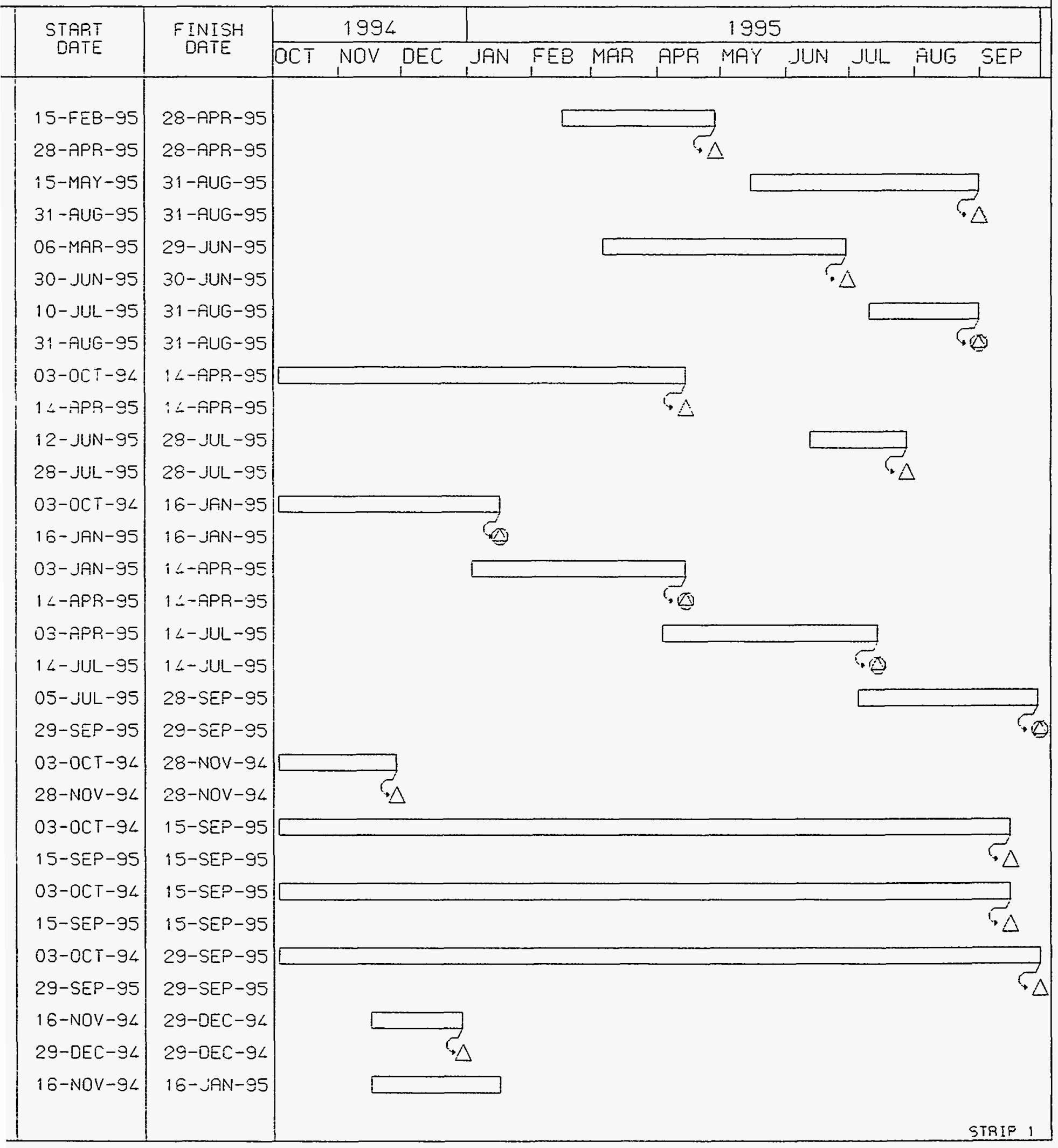




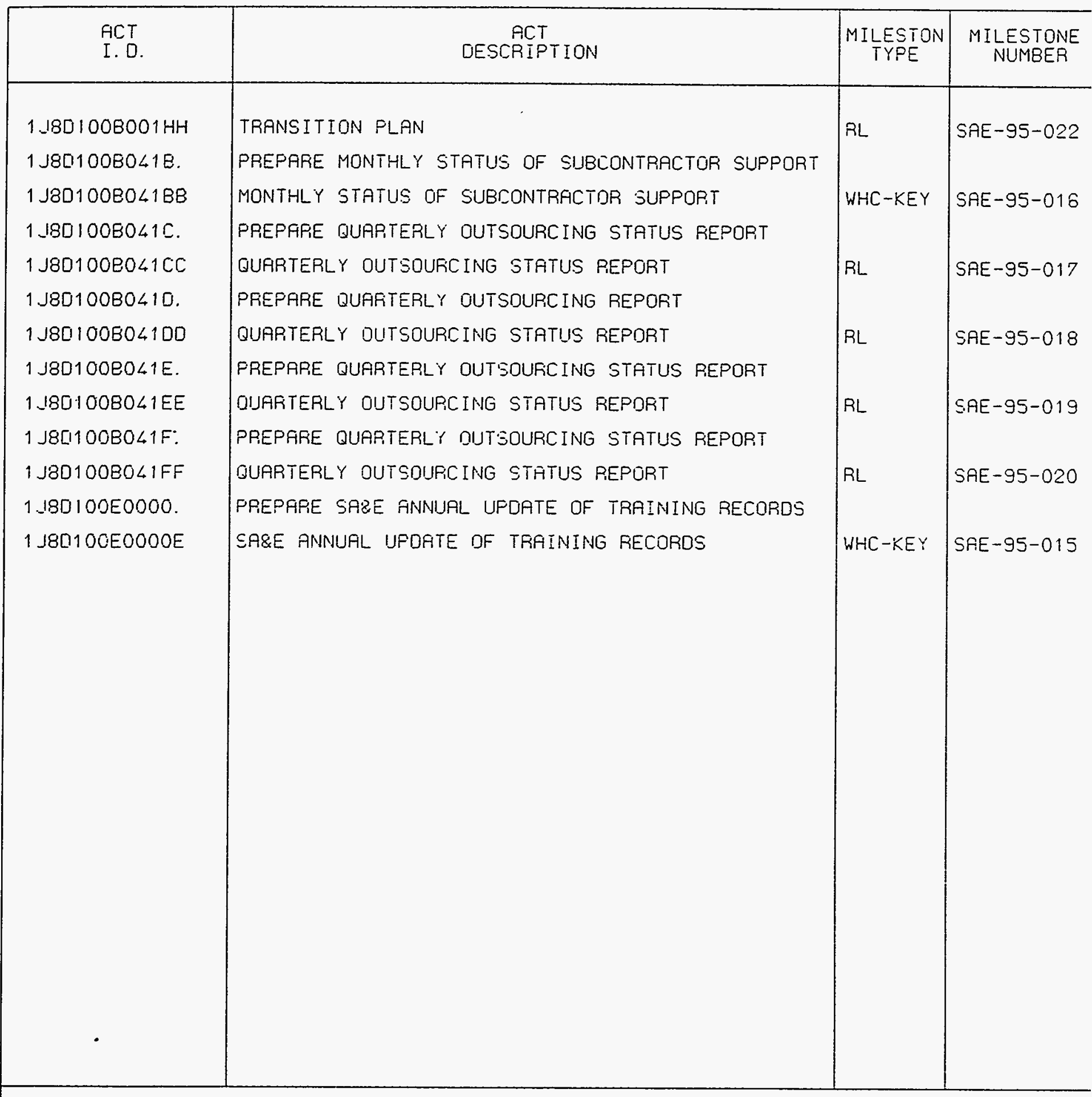


HC-SP-1116 REV 0

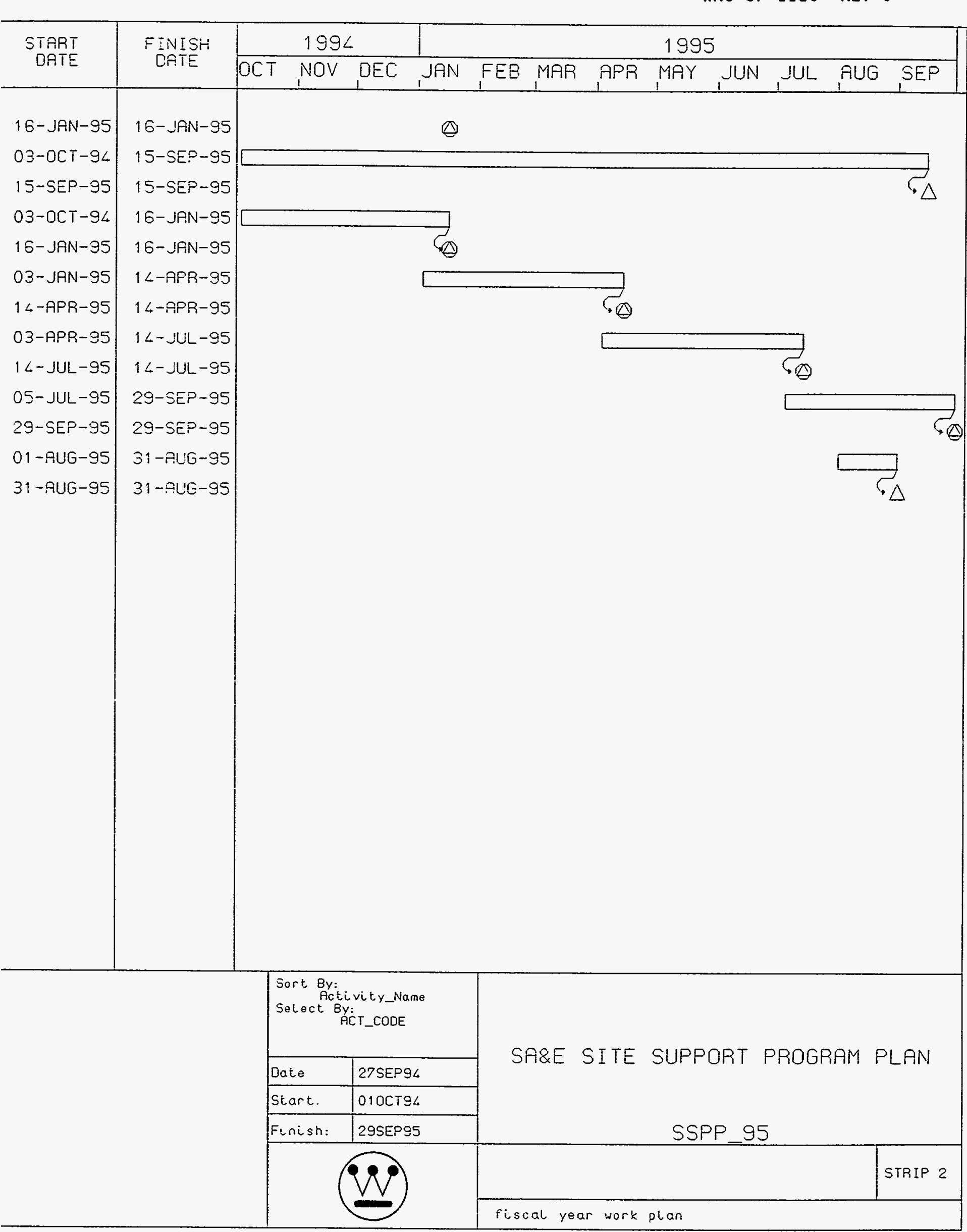

Overheads, Safety Analysis \& Engineering Page 2-11/2-12 


\section{WESTINGHOUSE HANFORD COMPANY F,Y-95 SITE SUPPORT PROGRAM PLAN RESOURCES WHC-SP-1116}

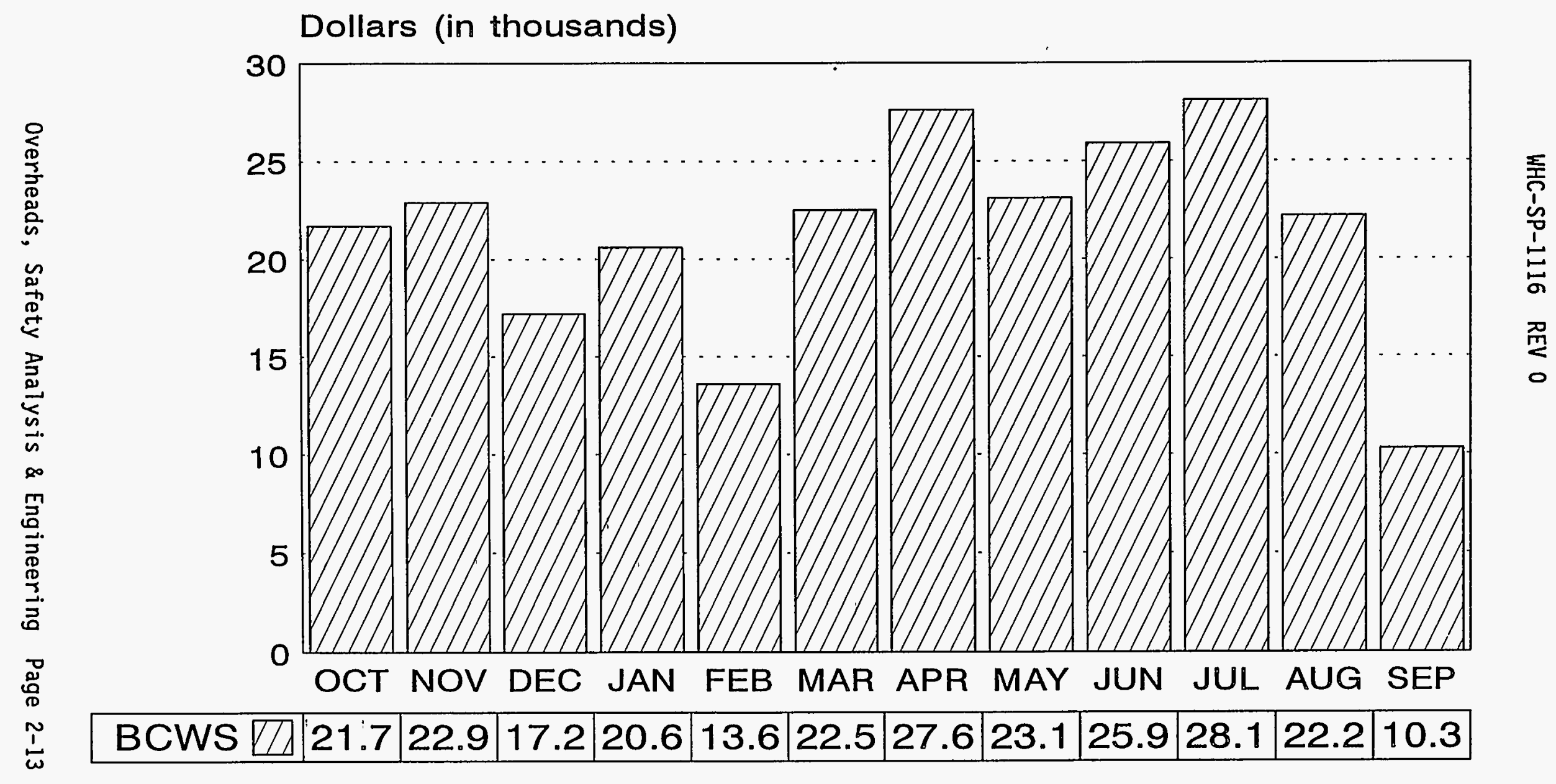


WHC-SP-1116 REV 0

This page intentionally left blank.

Overheads, Safety Analysis \& Engineering Page 2-14 
WHC-SP-1116 REV 0

\begin{tabular}{|c|c|c|c|}
\hline 2.C Cost Basel ine & \multirow{2}{*}{$\begin{array}{c}\text { Westinghouse Hanford Company } \\
\text { Overheads, Safety Analys is \& Engineering } \\
\text { SMS/WBS } 6.3 .5\end{array}$} & \multirow{2}{*}{\multicolumn{2}{|c|}{$\begin{array}{l}\text { FY } 1995 \\
\text { Site Support Program Plan } \\
\text { Date Prepared: } 9 / 14 / 94\end{array}$}} \\
\hline $\begin{array}{l}\text { 2. C. } 1 \text { Cost Baseline } \\
\text { by Program Element }\end{array}$ & & & \\
\hline \multicolumn{4}{|c|}{$\because$ Fiscal Year 1994 Cost Baseline (Dol7ars in Thousands) } \\
\hline WBS number & \multicolumn{2}{|l|}{ Title } & Total dollars \\
\hline 6.3 .5 & \multicolumn{2}{|c|}{$\begin{array}{l}\text { FY } 1994 \text { WHC Site reorganization terminated cost account plan } 132900 \\
\text { April 14, } 1994 \text {. Cost account plan } 13810 \text { was initiated, continuing safety } \\
\text { analysis work for the remainder of FY } 1994 \text {. Performance data unavailable. }\end{array}$} & \\
\hline \multicolumn{3}{|c|}{$\begin{array}{r}\text { FY } 1994 \text { Total for SMS/WBS } 6.3 .5 \text { (excludes company adders [G\&A and Common Support Pool } \\
{[\text { CSP]/Oversight] }}\end{array}$} & 4,190 \\
\hline \multicolumn{4}{|c|}{ Fiscal Year 1995 cost Baseline (Dollars in Thousands) } \\
\hline WBS number & \multicolumn{2}{|l|}{ Title } & Total dollars \\
\hline 6.3 .5 .1 & \multicolumn{2}{|l|}{ Management and Administration } & \\
\hline 6.3 .5 .1 .1 & \multicolumn{2}{|l|}{ Nondiscretionary Operating Costs } & 1,255 \\
\hline 6.3 .5 .1 .2 & \multicolumn{2}{|l|}{ 8D100 Management Activities } & 115 \\
\hline 6.3 .5 .1 .3 & \multicolumn{2}{|l|}{ Hourly Support } & 31 \\
\hline 6.3 .5 .1 .4 & \multicolumn{2}{|l|}{ Staff Moves } & 30 \\
\hline 6.3 .5 .2 & \multicolumn{2}{|l|}{ Program Management } & \\
\hline 6.3 .5 .2 .1 & \multicolumn{2}{|l|}{ Program Development and Implementation } & 288 \\
\hline 6.3 .5 .2 .2 & \multicolumn{2}{|l|}{ EFCOG Activities } & 20 \\
\hline 6.3 .5 .2 .3 & \multicolumn{2}{|l|}{ Professional Societies and Standards Groups } & 20 \\
\hline 6.3 .5 .2 .4 & \multicolumn{2}{|l|}{ Contracts Administration } & 181 \\
\hline 6.3 .5 .2 .5 & \multicolumn{2}{|l|}{ Corrective Action Management (CAM) Administration } & 0 \\
\hline
\end{tabular}

Overheads, Safety Analysis \& Engineering Page 2-15 
WHC-SP-1116 REV 0

\begin{tabular}{|c|c|c|c|}
\hline \multirow{2}{*}{$\begin{array}{l}\text { 2.C Cost Baseline } \\
\text { 2.C. } 1 \text { Cost Baseline } \\
\text { by Program Element }\end{array}$} & \multirow{2}{*}{$\begin{array}{l}\text { Westinghouse Hanford Company } \\
\text { overheads, Safety Analysis \& Engineering } \\
\text { SMS/WBS } 6.3 .5\end{array}$} & \multirow{2}{*}{\multicolumn{2}{|c|}{$\begin{array}{l}\text { FY } 1995 \\
\text { Site Support Program Plan } \\
\text { Date Prepared: } 9 / 14 / 94\end{array}$}} \\
\hline & & & \\
\hline 6.3 .5 .3 & \multicolumn{3}{|l|}{ Qualification and Training } \\
\hline 6.3 .5 .3 .1 & \multicolumn{2}{|l|}{ Mandatory Training } & 14 \\
\hline 6.3 .5 .3 .2 & \multicolumn{2}{|l|}{80100 Technical Training } & 4 \\
\hline 6.3 .5 .3 .3 & \multicolumn{2}{|l|}{ 80110 Technical Training } & 8 \\
\hline 6.3 .5 .3 .4 & \multicolumn{2}{|l|}{80120 Technical Training } & 3 \\
\hline 6.3 .5 .3 .5 & \multicolumn{2}{|l|}{80140 Technical Training } & 9 \\
\hline 6.3 .5 .3 .6 & \multicolumn{2}{|l|}{ 8D150 Technical Training } & 6 \\
\hline $6.3 \cdot 5.3 .7$ & \multicolumn{2}{|l|}{ 8D170 Technical Training } & 4 \\
\hline 6.3 .5 .4 & \multicolumn{2}{|l|}{ Emergent Work } & \\
\hline 6.3 .5 .4 .1 & \multicolumn{2}{|l|}{ Emergent Work } & 0 \\
\hline \multicolumn{3}{|c|}{ FY 1995 Total for SMS/WBS 6.3 .5 (excludes company adders [G\&A and CSP/Oversight]) } & 1,989 \\
\hline \multicolumn{4}{|c|}{ Fiscal Year 1996 Cost Baseline (Dollars in Thousands) } \\
\hline WBS number & \multicolumn{2}{|l|}{ Title } & Total dollars \\
\hline 6.3 .5 .1 & \multicolumn{2}{|l|}{ Management and Administration } & \\
\hline 6.3 .5 .1 .1 & \multicolumn{2}{|l|}{ Nondiscretionary Operating Costs } & 1,293 \\
\hline 6.3 .5 .1 .2 & \multicolumn{2}{|l|}{80100 Management Activities } & 118 \\
\hline 6.3 .5 .1 .3 & \multicolumn{2}{|l|}{ Hourly Support } & 32 \\
\hline 6.3 .5 .1 .4 & \multicolumn{2}{|l|}{ Staff Moves } & 31 \\
\hline
\end{tabular}

Overheads, Safety Analysis \& Engineering Page 2-16 
WHC-SP-1116 REV 0

\begin{tabular}{|c|c|c|c|}
\hline \multirow{2}{*}{$\begin{array}{l}\text { 2.C Cost Baseline } \\
\text { 2.C.1 Cost Baseline } \\
\text { by Program Element }\end{array}$} & \multirow{2}{*}{$\begin{array}{l}\text { Westinghouse Hanford Company } \\
\text { Overheads, Safety Analysis \& Engineering } \\
\text { SMS/WBS } 6.3 .5\end{array}$} & \multirow{2}{*}{\multicolumn{2}{|c|}{$\begin{array}{l}\text { FY } 1995 \\
\text { Site Support Program Plan } \\
\text { Date Prepared: } 9 / 14 / 94\end{array}$}} \\
\hline & & & \\
\hline 6.3 .5 .2 & \multicolumn{3}{|l|}{ Program Management } \\
\hline 6.3 .5 .2 .1 & \multicolumn{2}{|l|}{ Program Development and Implementation } & 242 \\
\hline 6.3 .5 .2 .2 & \multicolumn{2}{|l|}{ EFCOG Activities } & 21 \\
\hline $6.3 \cdot 5 \cdot 2.3$ & \multicolumn{2}{|l|}{ Professional Societies and Standards Groups } & 21 \\
\hline 6.3 .5 .2 .4 & \multicolumn{2}{|l|}{ Contracts Administration } & 186 \\
\hline 6.3 .5 .2 .5 & \multicolumn{2}{|l|}{ CAM Administration } & 0 \\
\hline 6.3 .5 .3 & \multicolumn{2}{|l|}{ Qualification and Training } & \\
\hline $6.3 \cdot 5 \cdot 3.1$ & \multicolumn{2}{|l|}{ Mandatory Training } & 15 \\
\hline $6.3 \cdot 5.3 .2$ & \multicolumn{2}{|l|}{80100 Technical Training } & 4 \\
\hline $6.3 \cdot 5 \cdot 3.3$ & \multicolumn{2}{|l|}{80110 Technical Training } & 8 \\
\hline $6.3 \cdot 5.3 .4$ & \multicolumn{2}{|l|}{ 8D120 Technical Training } & 3 \\
\hline $6.3 \cdot 5 \cdot 3.5$ & \multicolumn{2}{|l|}{$8 D 140$ Technical Training } & 9 \\
\hline 6.3 .5 .3 .6 & \multicolumn{2}{|l|}{ 8D150 Technical Training } & 6 \\
\hline $6.3 \cdot 5.3 .7$ & \multicolumn{2}{|l|}{ 8D170 Technical Training } & 4 \\
\hline 6.3 .5 .4 & \multicolumn{2}{|l|}{ Emergent Work } & \\
\hline 6.3 .5 .4 .1 & \multicolumn{2}{|l|}{ Emergent Work } & 0 \\
\hline \multicolumn{3}{|c|}{ FY 1996 Total for SMS/WBS 6.3 .5 (exc]udes company adders [G\&A and CSP/Oversight])] } & 1,993 \\
\hline
\end{tabular}




\begin{tabular}{|c|c|c|}
\hline 2.C Cost Baseline & \multirow{2}{*}{$\begin{array}{c}\text { Westinghouse Hanford Company } \\
\text { Overheads, Safety Analysis \& Engineering } \\
\text { SMS/WBS } 6.3 .5\end{array}$} & \multirow{2}{*}{$\begin{array}{l}\text { FY } 1995 \\
\text { Site Support Program Plan } \\
\text { Date Prepared: } 9 / 14 / 94\end{array}$} \\
\hline $\begin{array}{r}\text { 2.C.1 Cost Baseline } \\
\text { by Program Element }\end{array}$ & & \\
\hline \multicolumn{3}{|c|}{ Fiscal Year 1997 Cost Baseline (Dollars in Thousands) } \\
\hline WBS number & Title & Total dollars \\
\hline 6.3 .5 .1 & Management and Administration & \\
\hline 6.3 .5 .1 .1 & Nondiscretionary Operating Costs & 1,330 \\
\hline 6.3 .5 .1 .2 & 80100 Management Activities & 122 \\
\hline $6.3 .5 \cdot 1.3$ & Hourly Support & 33 \\
\hline 6.3 .5 .1 .4 & Staff Moves & 32 \\
\hline 6.3 .5 .2 & Program Management & \\
\hline 6.3 .5 .2 .1 & Program Development and Implementation & 250 \\
\hline 6.3 .5 .2 .2 & EFCOG Activities & 21 \\
\hline 6.3 .5 .2 .3 & Professional Societies and Standards Groups & 21 \\
\hline 6.3 .5 .2 .4 & Contracts Administration & 192 \\
\hline 6.3 .5 .2 .5 & CAM Administration & 0 \\
\hline 6.3 .5 .3 & Qualification and Training & \\
\hline $6.3 \cdot 5 \cdot 3.1$ & Mandatory Training & 15 \\
\hline $6.3 \cdot 5 \cdot 3.2$ & 8D100 Technical Training & 5 \\
\hline $6.3 \cdot 5 \cdot 3 \cdot 3$ & 80110 Technical Training & 8 \\
\hline 6.3 .5 .3 .4 & 80120 Technical Training & 3 \\
\hline 6.3 .5 .3 .5 & 80140 Technical Training & 9 \\
\hline
\end{tabular}


WHC-SP-1116 REV 0

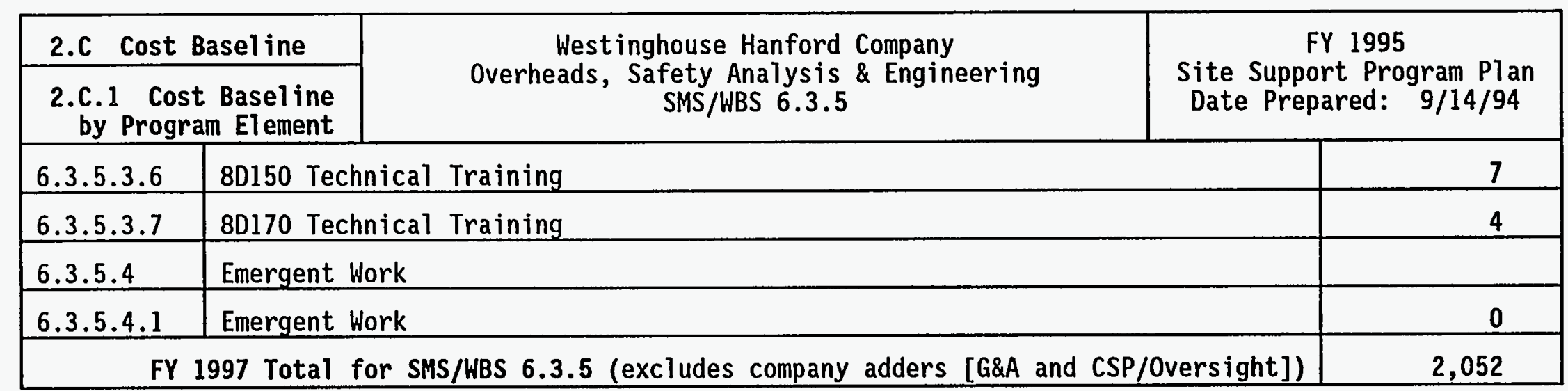


Westinghouse Hanford Company

2.C.2 Cost Basis
Overheads, Safety Analysis \& Engineering, SMS/WBS 6.3.5
FY 1995

Site Support Program Plan Date Prepared: 9/14/94

\subsubsection{Management and Administration}

The FY 1995 Nondiscretionary Operating Costs activities are estimated on the basis of FY 1994 historical data analysis for Site assessments and services plus three percent escalation for FY 1995 through FY 1997, in accordance with WHC guidance. The FY 1995 cost estimates for 8D100 Management and Hourly Support activities are definitively based because of WHC reorganization and departmental downsizing of support personnel. This level of support is expected to be reduced slightly in FY 1996 and FY 1997. The FY 1995 Staff Moves cost is estimated from FY 1993 and FY 1994 historical data and expectations for a planned move of personnel located at the ETC-1 Building to the $450 \mathrm{Hills}$ Building. It is anticipated that FY 1996 and FY 1997 Staff Moves activities will be minimal.

\subsubsection{Program Management}

The costs in FY 1995 for SA\&E Program Development and Implementation, Contracts Administration, and CAM Administration are estimated on the basis of planned, discreet milestones and FY 1994 historical data analysis of program documentation requirements specified by DOE-RL and WHC orders and procedures. It is assumed that these requirements will continue into FY 1996 and FY 1997 and are essential for the SA\&E department to manage its programs in a cost-effective manner.

Estimates for FY 1995 EFCOG Activities and Professional Societies and Standards Groups are based on historical data analysis and management's anticipated support requirement for WHC involvement in seminars, meetings, committees, and conferences. In FY 1996 and FY 1997, SA\&E will continue at near the same activity level as FY 1995. 


\begin{tabular}{|l|c|c|}
\hline 2.C.2 Cost Basis & $\begin{array}{c}\text { Hestinghouse Hanford Company } \\
\text { Safety Analysis \& Engineering } \\
\text { SMS/WBS } 6.3 .5\end{array}$ & $\begin{array}{c}\text { FY } 1995 \\
\text { Site Support Program Plan } \\
\text { Date Prepared: } 9 / 14 / 94\end{array}$ \\
\hline
\end{tabular}

\subsubsection{Qualifications and Training}

The cost basis for the FY 1995 SA\&E Mandatory Training activities has been developed from FY 1994 historical data analysis to ensure that SA\&E personnel receive required Hanford General Employee Training, Management Safety, and Drug-free-in-the-workplace Training, update refresher briefings for employees with special clearances, and job-specific seminars. The Technical Training cost for FY 1995 was developed from historical data analysis and parametric relationships, the number of SA\&E nonexempt personne 1, at $\$ 300$ each annual cost, and the number of exempt personnel, at $\$ 500$ each annual cost. Additional funding for unique individual training needs has been developed from historical data. FY 1996 and FY 1997 costs reflect FY 1995 cost plus escalation at three percent.

\subsubsection{Emergent Work}

Emergent Work cost basis will be estimated by utilization of historical, parametric, or definitive estimating techniques applicable subsequent to imposed, unbudgeted, and unplanned work scope. 
WHC-SP-1116 REV 0

\begin{tabular}{|c|c|c|c|c|c|c|c|c|}
\hline \multicolumn{3}{|c|}{$\begin{array}{l}\text { 2.C.3 Full-Time Equivalent Staff by Job } \\
\text { Description (Job Family Only after 1996) }\end{array}$} & \multicolumn{3}{|c|}{$\begin{array}{c}\text { Westinghouse Hanford Company } \\
\text { Overheads, Safety Analys is \& } \\
\text { Engineering, SMS/WBS } 6.3 .5\end{array}$} & \multicolumn{3}{|c|}{$\begin{array}{c}\text { FY } 1995 \\
\text { Site Support Program Plan } \\
\text { Date Prepared: } 9 / 14 / 94\end{array}$} \\
\hline & 1994 & 1995 & 1996 & 1997 & 1998 & 1999 & 2000 & 2001 \\
\hline \multicolumn{9}{|l|}{ JOB FAMILYY } \\
\hline \multicolumn{9}{|l|}{ Job category } \\
\hline MANAGERS & 3.40 & 2.50 & 2.00 & 2.00 & 2.00 & 2.00 & 2.00 & 2.00 \\
\hline \multicolumn{9}{|l|}{ First line } \\
\hline \multicolumn{9}{|l|}{ General/executive } \\
\hline Project/Program & 3,40 & 2.00 & 2.00 & & & & & \\
\hline other & & 0.50 & & & & & & \\
\hline ENGI MEERS & 1.00 & $\therefore$ & $\because$ & $\therefore \quad \therefore$ & $\therefore \quad \cdots$ & ४ै & खे & 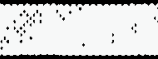 \\
\hline \multicolumn{9}{|l|}{ Chemical } \\
\hline \multicolumn{9}{|l|}{ Civil } \\
\hline \multicolumn{9}{|l|}{ Computer } \\
\hline \multicolumn{9}{|l|}{ Electrical } \\
\hline \multicolumn{9}{|l|}{ Environmental } \\
\hline \multicolumn{9}{|l|}{ Industrial } \\
\hline \multicolumn{9}{|l|}{ Mechanical } \\
\hline Nuclear & 1.00 & & & & & & & \\
\hline \multicolumn{9}{|l|}{ Petroleum/Mining } \\
\hline \multicolumn{9}{|l|}{ Plant } \\
\hline \multicolumn{9}{|l|}{ Qual ity Control } \\
\hline \multicolumn{9}{|l|}{ Safety } \\
\hline \multicolumn{9}{|l|}{ other } \\
\hline \multicolumn{9}{|l|}{ SCIENTISTS } \\
\hline \multicolumn{9}{|l|}{ Chemists } \\
\hline Enviromental & & & & & & & & \\
\hline
\end{tabular}

Overheads, Safety Analysis \& Engineering Page 2-22 
WHC-SP-1116, REV 0

\begin{tabular}{|c|c|c|c|c|c|c|c|c|}
\hline \multicolumn{3}{|c|}{$\begin{array}{l}\text { 2.C.3 Full-Time Equivalent Staff by Job } \\
\text { Description (Job Family Only after 1996) }\end{array}$} & \multicolumn{3}{|c|}{$\begin{array}{c}\text { West inghouse Hanford Company } \\
\text { Overheads, Safety Analys is \& } \\
\text { Engineering, SMS/WBS 6.3.5 }\end{array}$} & \multicolumn{3}{|c|}{$\begin{array}{l}\text { FY } 1995 \\
\text { Site Support Program P1an } \\
\text { Date Prepared: } \quad 9 / 14 / 94\end{array}$} \\
\hline & 1994 & 1995 & 1996. & 1997 & 1998 & $1999 \$$ & 2000 & 2001 \\
\hline \multicolumn{9}{|l|}{ Geologists } \\
\hline \multicolumn{9}{|l|}{ Life } \\
\hline \multicolumn{9}{|l|}{ Material } \\
\hline \multicolumn{9}{|l|}{ Mathematicians } \\
\hline \multicolumn{9}{|l|}{ Physicists } \\
\hline \multicolumn{9}{|l|}{ Social } \\
\hline \multicolumn{9}{|l|}{ other } \\
\hline ADMIN/OTHER PROFESSIONALS & 1.10 & $\therefore 1.00$ & 1.00 & $\therefore \quad 1.00$ & 1.00 & 1.000 & $1.00 \%$ & 1.00 \\
\hline \multicolumn{9}{|l|}{ Accountant/auditor } \\
\hline \multicolumn{9}{|l|}{ Architect } \\
\hline \multicolumn{9}{|l|}{ Buyers/procurement } \\
\hline \multicolumn{9}{|l|}{ Communications } \\
\hline \multicolumn{9}{|l|}{ Compl iance inspectors } \\
\hline \multicolumn{9}{|l|}{ Computer System Anal } \\
\hline Cost Est/planner/sch & 1.10 & 1.00 & 1.00 & & & & & \\
\hline \multicolumn{9}{|l|}{ Heal th Physics } \\
\hline \multicolumn{9}{|l|}{ Industrial Hygiene } \\
\hline \multicolumn{9}{|l|}{ Lawyers } \\
\hline \multicolumn{9}{|l|}{ Personnel/Labor Rela } \\
\hline \multicolumn{9}{|l|}{ Physicians } \\
\hline \multicolumn{9}{|l|}{ Physician Assis/Nurs } \\
\hline \multicolumn{9}{|l|}{ Safeguard \& Security } \\
\hline \multicolumn{9}{|l|}{ Tech Writers \& Edit } \\
\hline Trainers & & & & & & & & \\
\hline
\end{tabular}

Overheads, Safety Analysis \& Engineering Page 2-23 
WHC-SP-1116 REV 0

\begin{tabular}{|c|c|c|c|c|c|c|c|c|}
\hline \multicolumn{3}{|c|}{$\begin{array}{l}\text { 2.C.3 Full-Time Equivalent Staff by Job } \\
\text { Description (Job Family Only after 1996) }\end{array}$} & \multicolumn{3}{|c|}{$\begin{array}{c}\text { Westinghouse Hanford Company } \\
\text { Overheads, Safety Analysis \& } \\
\text { Engineering, SMS/WBS } 6.3 .5\end{array}$} & \multicolumn{3}{|c|}{$\begin{array}{c}\text { FY } 1995 \\
\text { Site Support Program Plan } \\
\text { Date Prepared: } 9 / 14 / 94\end{array}$} \\
\hline & 1994 & 1995 & 1996 & 1997 & 1998 & $1999^{\circ}$ & 2000 & 2001 \\
\hline \multicolumn{9}{|l|}{ other } \\
\hline \multicolumn{9}{|l|}{ JOB FAMILY } \\
\hline \multicolumn{9}{|l|}{ Job category } \\
\hline GEN ADM/SECRETARY/CLERK & 16.40 & 6.05 & 5.55 & 5.55 & 5.55 & 5.55 & 5.55 & 5.55 \\
\hline Admin Assistants & 8.70 & 2.55 & 2.55 & & & & & \\
\hline office Clerks (Gen) & 5.30 & 2.40 & 2.40 & & & & & \\
\hline \multicolumn{9}{|l|}{ office clerks (Special) } \\
\hline Secretaries & 2.40 & 1.10 & 0.60 & & & & & \\
\hline \multicolumn{9}{|l|}{ Typist/Word Process } \\
\hline \multicolumn{9}{|l|}{ other } \\
\hline TECHNICIANS & & & & 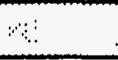 & $\because$ & $\ldots \ldots$ & $\therefore: \ldots$ & \\
\hline \multicolumn{9}{|l|}{ Computer Oper/Coder } \\
\hline \multicolumn{9}{|l|}{ Drafters } \\
\hline \multicolumn{9}{|l|}{ Engrs/Tech } \\
\hline \multicolumn{9}{|l|}{ Envir. Sci Technicians } \\
\hline \multicolumn{9}{|l|}{ Heal th Phys. Technic. } \\
\hline \multicolumn{9}{|l|}{ Indus. Saf/Heal th Tech } \\
\hline \multicolumn{9}{|l|}{ Instru/Control Tech } \\
\hline \multicolumn{9}{|l|}{ Lab. Technicians } \\
\hline \multicolumn{9}{|l|}{ Media Technicians } \\
\hline \multicolumn{9}{|l|}{ Survey/Map Tech } \\
\hline \multicolumn{9}{|l|}{ other } \\
\hline \multicolumn{9}{|l|}{ CRAFTS } \\
\hline Carpenters & & & & & & & & \\
\hline
\end{tabular}

Overheads, Safety Analysis \& Engineering Page 2-24 
WHC-SP-1116 REV 0

\begin{tabular}{|c|c|c|c|c|c|c|c|c|}
\hline \multicolumn{3}{|c|}{$\begin{array}{l}\text { 2.C.3 Ful1-Time Equivalent Staff by Job } \\
\text { Description (Job Family Only after 1996) }\end{array}$} & \multicolumn{3}{|c|}{$\begin{array}{c}\text { Westinghouse Hanford Company } \\
\text { Overheads, Safety Analys is \& } \\
\text { Engineering, SMS/WBS } 6.3 .5\end{array}$} & \multicolumn{3}{|c|}{$\begin{array}{c}\text { FY } 1995 \\
\text { Site Support Program Plan } \\
\text { Date Prepared: } \quad 9 / 14 / 94\end{array}$} \\
\hline & $1994:$ & 1995 & 1996 & 1997 & 1998 & $1999 \%$ & 2000 & 2001: \\
\hline \multicolumn{9}{|l|}{ Electricians } \\
\hline \multicolumn{9}{|l|}{ HVAC } \\
\hline \multicolumn{9}{|l|}{ Machinists } \\
\hline \multicolumn{9}{|l|}{ Masons } \\
\hline \multicolumn{9}{|l|}{ Millwrights } \\
\hline \multicolumn{9}{|l|}{ Painters } \\
\hline \multicolumn{9}{|l|}{ Plumbers/Pipefitters } \\
\hline \multicolumn{9}{|c|}{ Struct/Metal Horkers } \\
\hline \multicolumn{9}{|c|}{ Vehic./Mob Equip Mech } \\
\hline \multicolumn{9}{|c|}{ Helders } \\
\hline \multicolumn{9}{|l|}{ other } \\
\hline OPERATORS & & & . & & $\therefore$ & 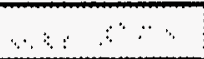 & . & $4 \ldots$ \\
\hline \multicolumn{9}{|l|}{ Chemical system } \\
\hline \multicolumn{9}{|l|}{ Drillers } \\
\hline \multicolumn{9}{|l|}{ Lt. Vehicle Drivers } \\
\hline \multicolumn{9}{|c|}{ Material Moving Equip } \\
\hline \multicolumn{9}{|l|}{ Nuclear Plant } \\
\hline \multicolumn{9}{|c|}{ Utilities Waste Proces } \\
\hline \multicolumn{9}{|l|}{ other } \\
\hline \multicolumn{9}{|l|}{ LABOR \& GEN WORKERS } \\
\hline \multicolumn{9}{|l|}{ Firefighters } \\
\hline \multicolumn{9}{|l|}{ Food Service } \\
\hline \multicolumn{9}{|l|}{ Hand/Help Lab Gen } \\
\hline Hand/Help Lab Spec & & & & & & & & \\
\hline
\end{tabular}

Overheads, Safety Analysis \& Engineering Page 2-25 
WHC-SP-1116 REV 0

\begin{tabular}{|c|c|c|c|c|c|c|c|}
\hline \multicolumn{2}{|c|}{$\begin{array}{l}\text { 2.C.3 Ful1-Time Equivalent Staff by Job } \\
\text { Description (Job Family Only after 1996) }\end{array}$} & \multicolumn{3}{|c|}{$\begin{array}{l}\text { Westinghouse Hanford Company } \\
\text { Overheads, Safety Analys is \& } \\
\text { Engineering, SMS/WBS } 6.3 .5\end{array}$} & \multicolumn{3}{|c|}{$\begin{array}{l}\text { FY } 1995 \\
\text { Site Support Program Plan } \\
\text { Date Prepared: } 9 / 14 / 94\end{array}$} \\
\hline 1994 & 1995 & 1996 & 1997 & 1998 & 1999 & 2000 & 2001 \\
\hline \multicolumn{8}{|l|}{ Jani tors/Cleaners } \\
\hline \multicolumn{8}{|l|}{ Laundry Workers } \\
\hline \multicolumn{8}{|l|}{ Security Guards } \\
\hline \multicolumn{8}{|l|}{ Other } \\
\hline Totals & 9.55 & 8.55 & 8.55 & 8.55 & 8.55 & 8.55 & 8.55 \\
\hline
\end{tabular}









\begin{tabular}{|c|c|c|}
\hline $\begin{array}{c}1 \mathrm{~J} 8010 \\
\text { COST ACCOUNT }\end{array}$ & $W H C-S P-1116$ REV 0 & \\
\hline $\begin{array}{l}\text { Work Breakdown } \\
\text { Structure } \\
\text { Dictionary }\end{array}$ & $\begin{array}{l}\text { Westinghouse Hanford Company } \\
\text { (Overheads, Safety Analysis and Engineering) } \\
\text { Part II - Element Definition }\end{array}$ & $\begin{array}{l}\text { FY } 1995 \text { SSPP } \\
\text { Revision \# } 0\end{array}$ \\
\hline
\end{tabular}

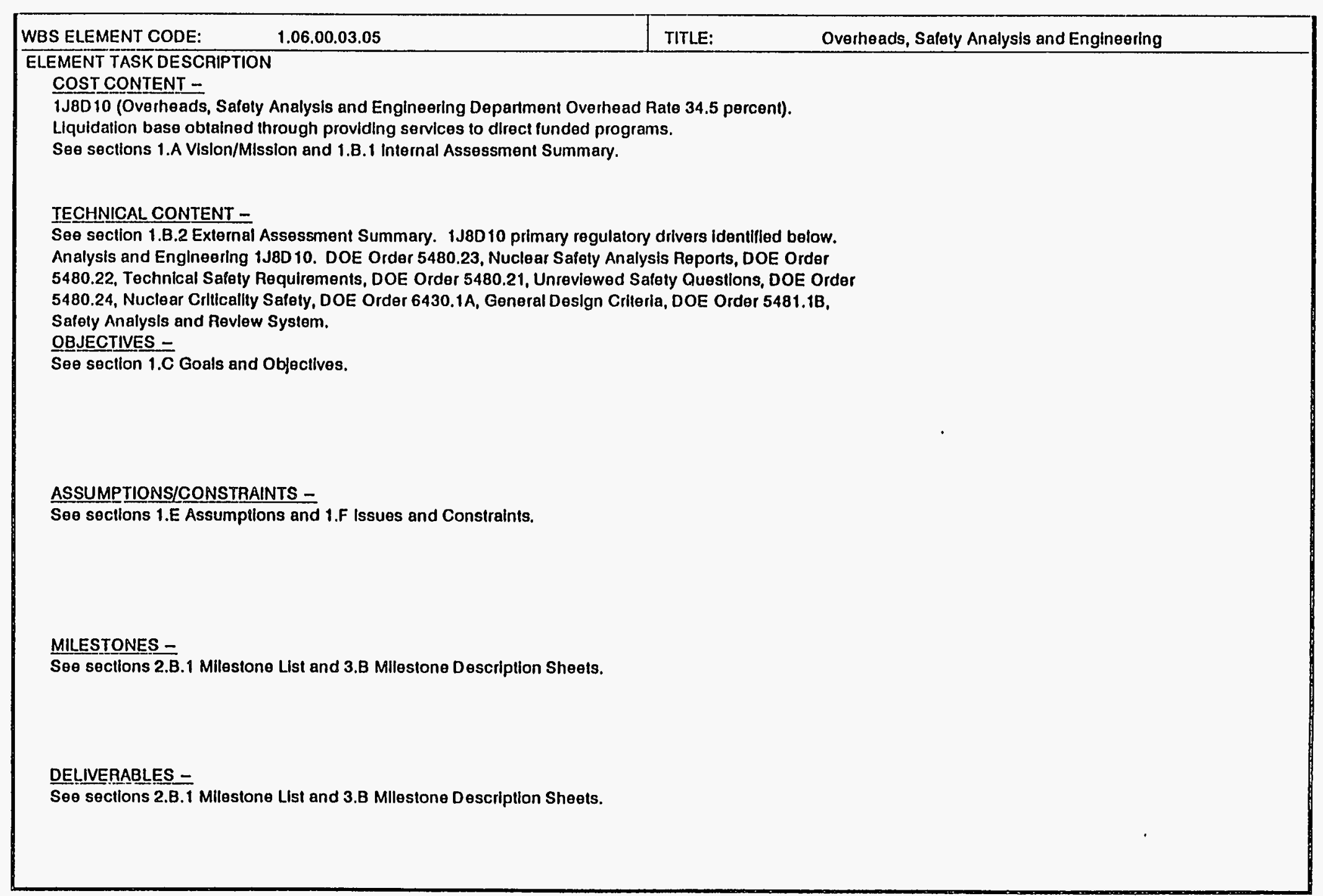

Overheads, Safety Analysis \& Engineering Page 3-2 
WHC-SP-1116 REV 0

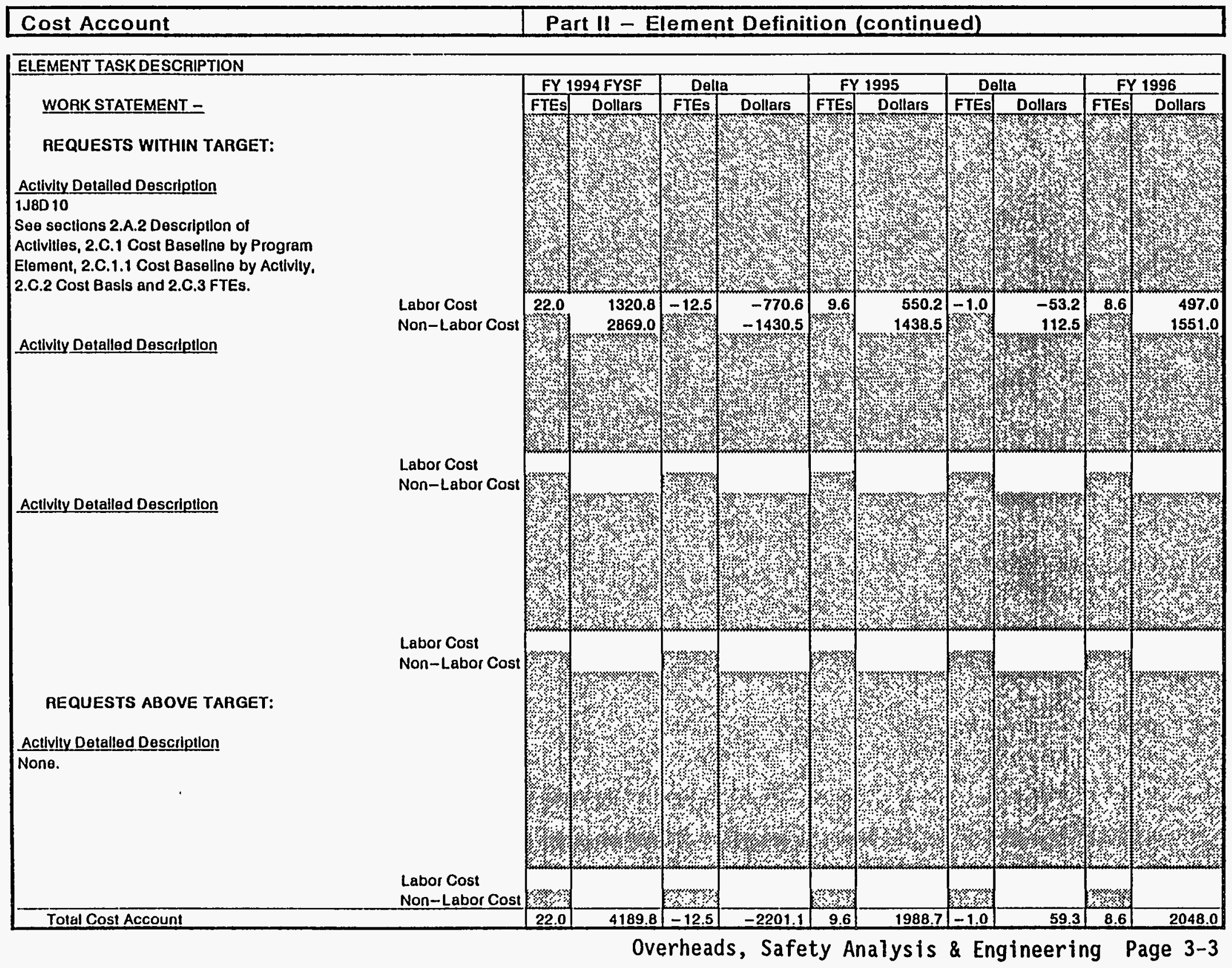




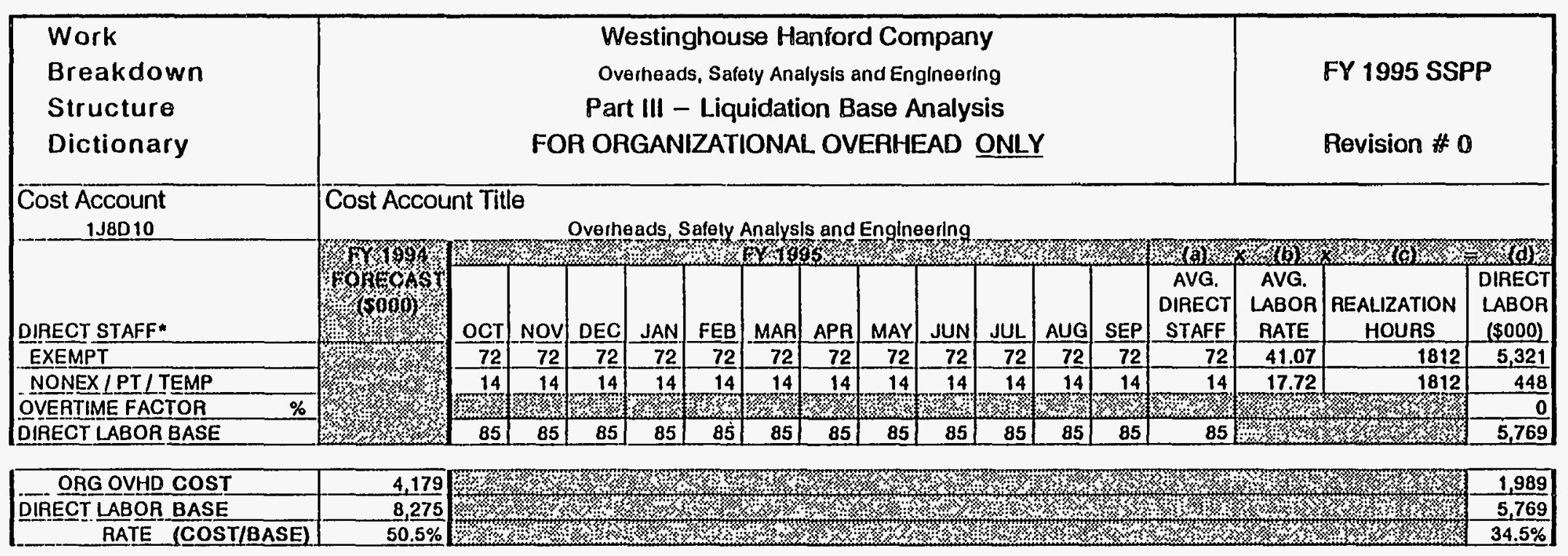

- Direct staft is staff that charges labor to charge codes on which organizational overhead is applled. 


\section{B \\ Westinghouse Hanford Company \\ MILESTONE DESCRIPTION SHEET}

\begin{tabular}{|c|c|c|c|}
\hline \multicolumn{3}{|c|}{$\begin{array}{l}\text { Title: } \\
\text { Safety Analysis \& Engineering FY } 1995 \text { DOH Mid-Year Review }\end{array}$} & $\begin{array}{l}\text { Date: } \\
8 / 30 / 94\end{array}$ \\
\hline \multicolumn{3}{|c|}{$\begin{array}{l}\text { Assigned To: } \\
\text { SA\&E/PDI (E.P. DiVincenzo/S.M. Godfrey) } \\
\text { Program WBS Designator: }\end{array}$} & CIN: \\
\hline \multicolumn{3}{|c|}{$\begin{array}{l}\text { Program WBS Designator: } \\
6.3 .5 .2 .1 \text { (TPCN\# J8D1E) } \\
\end{array}$} & $\begin{array}{l}\text { Due Date: } \\
4 / 28 / 95\end{array}$ \\
\hline \multicolumn{3}{|c|}{ Control Number: SAE-95-001 } & Rev: 0 \\
\hline $\begin{array}{l}\text { MILESTONE TYPE: } \\
\square \text { DOE-HQ } \\
\square \text { DOE-RL } \\
\text { CNTR }\end{array}$ & $\begin{array}{l}\text { DIVISION: } \\
\square \text { State } \\
\square \text { Federal } \\
\square \text { DOE } \\
\square \text { RCRA } \\
\square \text { TPA NUMBER } \\
\text { WHC Budgets } \\
\end{array}$ & $\begin{array}{ll} & \text { DELIVERABLE: } \\
\square & \text { Report } \\
\square & \text { Letter } \\
\square & \text { Drawings } \\
\text { Other (specify) } \\
\text { Budget Review }\end{array}$ & $\begin{array}{ll} & \text { ADDRESS TO: } \\
\square & \text { DOE-HQ } \\
\text { DOE-RL } \\
\text { Other (specify) } \\
\text { SA\&E Program } \\
\text { Participants }\end{array}$ \\
\hline
\end{tabular}

Milestone Description:

Prepare Safety Analysis \& Engineering mid-year review data.

Description of what constitutes completion of this milestone:

Issue written guidance to program participants by April 28, 1995 (this could include revised budgets, change notices, etc. as required).

Copies of completed milestone deliverables will be submitted to SA\&E Program Development and Implementation (8D170) manager.

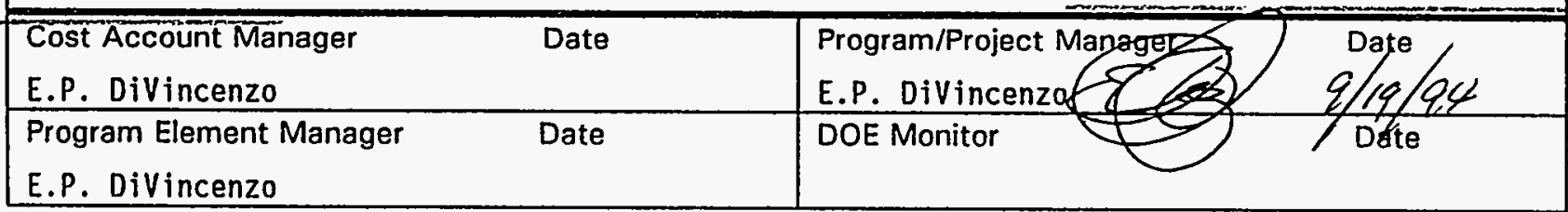


HHC-SP-1116 REV 0

\begin{tabular}{|c|c|c|c|}
\hline \multicolumn{4}{|c|}{$\begin{array}{l}\text { Westinghouse Hanford Company } \\
\text { MILESTONE DESCRIPTION SHEET }\end{array}$} \\
\hline \multicolumn{3}{|c|}{$\begin{array}{l}\text { Title: } \\
\text { Safety Analysis \& Engineering FY } 1996 \text { DOH Budget }\end{array}$} & $\begin{array}{l}\text { Date: } \\
8 / 30 / 94\end{array}$ \\
\hline \multicolumn{3}{|c|}{$\begin{array}{l}\text { Assigned To: } \\
\text { SA\&E/PDI (E.P. DiVincenzo/S.M. Godfrey) }\end{array}$} & CIN: \\
\hline \multicolumn{3}{|l|}{$\begin{array}{l}\text { Program WBS Designator: } \\
6.3 .5 .2 .1 \text { (TPCN\# J8D1E) }\end{array}$} & $\begin{array}{l}\text { Due Date: } \\
8 / 31 / 95\end{array}$ \\
\hline \multicolumn{3}{|c|}{ Control Number: SAE-95-002 } & Rev: 0 \\
\hline $\begin{array}{l}\text { MILESTONE TYPE: } \\
\square \text { DOE-HQ } \\
\square \text { DOE-RL } \\
\text { CNTR }\end{array}$ & $\begin{array}{l}\text { DIVISION: } \\
\square \text { State } \\
\square \text { Federal } \\
\square \text { DOE } \\
\square \text { RCRA } \\
\square \text { TPA NUMBER } \\
\text { WHC Budgets } \\
\end{array}$ & 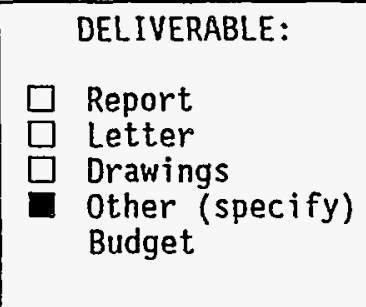 & $\begin{array}{l}\text { ADDRESS TO: } \\
\square \text { DOE-HQ } \\
\square \text { DOE-RL } \\
\text { Other (specify) } \\
\text { OSBRB }\end{array}$ \\
\hline \multicolumn{4}{|c|}{$\begin{array}{l}\text { Milestone Description: } \\
\text { Prepare Safety Analysis \& Engineering FY } 1996 \text { Department Overhead Budget 1J8D10. }\end{array}$} \\
\hline \multicolumn{4}{|c|}{$\begin{array}{l}\text { Description of what constitutes completion of this milestone: } \\
\text { Complete preparation of the Safety Analys is \& Engineering Department Overhead Budget and } \\
\text { submit for the OSBRB review by August } 31,1995 \text {. } \\
\text { Copies of completed milestone deliverables will be submitted to SA\&E Program Development } \\
\text { and Implementation (8D170) manager. }\end{array}$} \\
\hline $\begin{array}{l}\text { Cost Account Manager } \\
\text { E.P. DiVincenzo }\end{array}$ & Date & $\begin{array}{l}\text { Program/Project Manag } \\
\text { E.P. DiVincenzo }\end{array}$ & 23 atcelay \\
\hline $\begin{array}{l}\text { Program Element Manager } \\
\text { E.P. DiVincenzo }\end{array}$ & Date & DOE Monitor & Date \\
\hline
\end{tabular}

Overheads, Safety Analysis \& Engineering Page 3-6 
WHC-SP-1116 REV 0

\begin{tabular}{|c|c|c|c|}
\hline 3. B & \multicolumn{2}{|c|}{$\begin{array}{l}\text { Westinghouse Hanford Company } \\
\text { MILESTONE DESCRIPTION SHEET }\end{array}$} & \\
\hline \multicolumn{3}{|c|}{$\begin{array}{l}\text { Title: Draft of Safety Analysis \& Engineering FY } 1996 \text { Site Support } \\
\text { Program Plan/Multi-Year Program Plan }\end{array}$} & $\begin{array}{l}\text { Date: } \\
8 / 30 / 94\end{array}$ \\
\hline \multicolumn{3}{|c|}{$\begin{array}{l}\text { Assigned To: } \\
\text { SA\&E/PDI (E.P. DiVincenzo/G.A. Tarcza) } \\
\text { Program WBS Desionator }\end{array}$} & CIN: \\
\hline \multicolumn{3}{|c|}{ Program WBS Designator: } & $\begin{array}{l}\text { Due Date: } \\
6 / 30 / 95\end{array}$ \\
\hline \multicolumn{3}{|c|}{ Control Number: SAE-95-003 } & Rev: 0 \\
\hline $\begin{array}{l}\text { MILESTONE TYPE: } \\
\square \text { DOE-HQ } \\
\square \text { DOE-RL } \\
\text { CNTR }\end{array}$ & $\begin{array}{ll} & \text { DIVISION: } \\
\square & \text { State } \\
\square & \text { Federal } \\
\text { DOE } \\
\square \text { RCRA } \\
\square \text { TPA NUMBER }\end{array}$ & 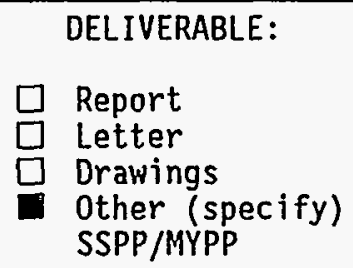 & $\begin{array}{l}\text { ADDRESS TO: } \\
\text { DOE-HQ } \\
\text { DOE-RL } \\
\text { Other (specify) } \\
\text { WHC } \\
\text { distribution }\end{array}$ \\
\hline
\end{tabular}

Milestone Description:

Complete the draft of the Safety Analysis \& Engineering Site Support Program Plan/MultiYear Program Plan.

Description of what constitutes completion of this milestone:

Prepare the draft Safety Analysis \& Engineering Site Support Program PIan for FY 1996 and submit to DOE-RL for review. PI an should include current Work Breakdown Structure and dictionary, milestone description sheets, schedules for milestones and proposed budget. Includes multi-year program planning. Final is due August 31,1995 . Submit to DOE-RL for initial review.

Copies of completed milestone deliverables will be submitted to SA\&E Program Development and Implementation (8D170) manager.

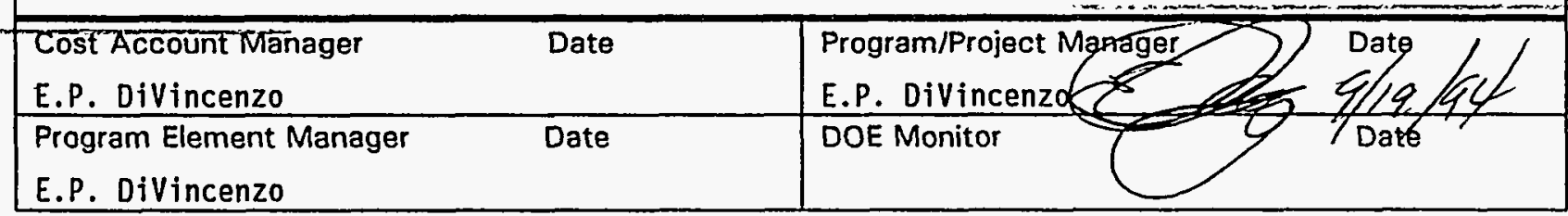


WHC-SP-1116 REV 0

\begin{tabular}{|c|c|c|c|}
\hline 3. $B$ & \multicolumn{2}{|c|}{$\begin{array}{l}\text { Westinghouse Hanford Company } \\
\text { MILESTONE DESCRIPTION SHEET }\end{array}$} & \\
\hline \multicolumn{3}{|c|}{$\begin{array}{l}\text { Title: Safety Analysis \& Engineering FY } 1996 \text { Site Support Program } \\
\text { Plan/Multi-Year Program Plan }\end{array}$} & $\begin{array}{l}\text { Date: } \\
8 / 30 / 94\end{array}$ \\
\hline \multicolumn{3}{|c|}{$\begin{array}{l}\text { Assigned To: } \\
\text { SA\&E/PDI (E.P. DiVincenzo/G.A. Tarcza) }\end{array}$} & CIN: \\
\hline \multicolumn{3}{|c|}{ 6.3.5.2.1 (TPCN\# J8D1E) } & $\begin{array}{l}\text { Due Date: } \\
8 / 31 / 95\end{array}$ \\
\hline \multicolumn{3}{|c|}{ Control Number: SAE-95-004 } & Rev: 0 \\
\hline $\begin{array}{l}\text { MILESTONE TYPE: } \\
\square \text { DOE-HQ } \\
\text { DOE-RL } \\
\text { CNTR }\end{array}$ & $\begin{array}{ll} & \text { DIVISION: } \\
& \text { State } \\
\square & \text { Federal } \\
\text { DOE } \\
\text { DCRA } \\
\square \text { RCRA NUMBER }\end{array}$ & $\begin{array}{l}\text { DELIVERABLE: } \\
\square \text { Report } \\
\square \text { Letter } \\
\square \text { Drawings } \\
\text { Other (specify) } \\
\text { SSPP/MYPP }\end{array}$ & $\begin{array}{l}\text { ADDRESS TO: } \\
\text { DOE-HQ } \\
\text { DOE-RL } \\
\text { Other (specify) } \\
\text { WHC } \\
\text { distribution }\end{array}$ \\
\hline
\end{tabular}

Milestone Description:

(Milestone SAE-95-003 rolls up to support this milestone.)

Complete final Safety Analysis \& Engineering Site Support Program PIan.

Description of what constitutes completion of this milestone:

Complete and submit for approval the FY 1996 Site Support Program P1an.

Copies of completed milestone deliverables will be submitted to SA\&E Program Development and Implementation (80170) manager.

\begin{tabular}{|c|c|c|c|}
\hline $\begin{array}{l}\text { Cost Account Manager } \\
\text { E.P. DiVincenzo }\end{array}$ & Date & $\begin{array}{l}\text { Program/Project Man } \\
\text { E.P. DiVincenzo }\end{array}$ & $\begin{array}{l}\text { Date } \\
\text { gleofact }\end{array}$ \\
\hline $\begin{array}{l}\text { Program Element Manager } \\
\text { E.P. DiVincenzo }\end{array}$ & Date & $\begin{array}{l}\text { DOE Monitor } \\
\text { M.R. Moreno }\end{array}$ & $9 / 20 / 94$ \\
\hline
\end{tabular}




\begin{tabular}{ll}
\hline 3.B Westinghouse Hanford Company \\
MILESTONE DESCRIPTION SHEET
\end{tabular}

\begin{tabular}{|c|c|c|c|}
\hline \multicolumn{3}{|c|}{$\begin{array}{l}\text { Title: Automated Data Processing Equipment Mid-Year Statement of } \\
\text { Strategy for FY } 1995\end{array}$} & $\begin{array}{l}\text { Date: } \\
8 / 30 / 94\end{array}$ \\
\hline \multicolumn{3}{|c|}{$\begin{array}{l}\text { Assigned To: } \\
\text { SA\&E/PDI (E.P. DiVincenzo/G.A. Tarcza) }\end{array}$} & CIN: \\
\hline \multicolumn{3}{|c|}{$\begin{array}{l}\text { Program WBS Designator: } \\
\text { 6.3.5.2.1 (TPCN\# J8D1E) }\end{array}$} & $\begin{array}{l}\text { Due Date: } \\
4 / 14 / 95\end{array}$ \\
\hline \multicolumn{3}{|c|}{ Control Number: SAE-95-005 } & Rev: 0 \\
\hline $\begin{array}{l}\text { MILESTONE TYPE: } \\
\square \text { DOE-HQ } \\
\square \text { DOE-RL. } \\
\text { CNTR }\end{array}$ & $\begin{aligned} & \text { DIVISION: } \\
& \square \text { State } \\
& \square \text { Federal } \\
& \square \text { DOE } \\
& \square \text { RCRA } \\
& \square \text { TPA NUMBER } \\
&\end{aligned}$ & $\begin{array}{l}\text { DELIVERABLE: } \\
\square \text { Report } \\
\square \text { Letter } \\
\square \text { Drawings } \\
\text { Other (specify) } \\
\text { ADPE Statement } \\
\text { of Strategy }\end{array}$ & 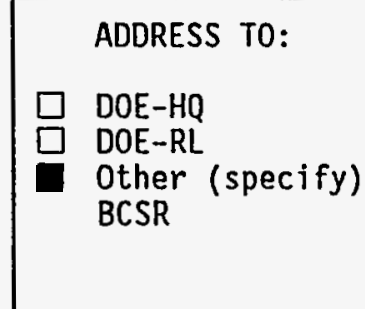 \\
\hline
\end{tabular}

Milestone Description:

Identify Safety Analysis \& Engineering Automated Data Processing Equipment Mid-Year Statement of Strategy for FY 1995.

Description of what constitutes completion of this milestone:

Prepare letter to identify Safety Analysis \& Engineering Statement of Strategy for hardware and software purchases for FY 1995 and submit to BCS Richland, Inc.

Copies of completed milestone deliverables will be submitted to SA\&E Program Development and Implementation (80170) manager.

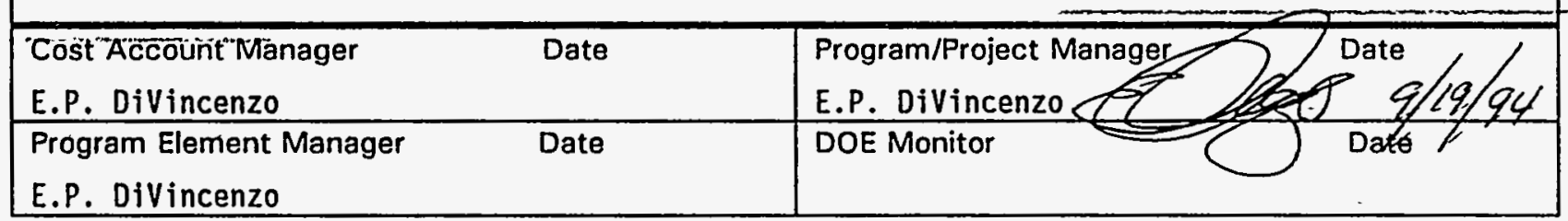


WHC-SP-1116 REV 0

3. B

Westinghouse Hanford Company MILESTONE DESCRIPTION SHEET

\begin{tabular}{|c|c|c|c|}
\hline \multicolumn{3}{|c|}{$\begin{array}{l}\text { Title: } \\
\text { Automated Data Processing Equipment Procurement P1an for FY } 1996\end{array}$} & $\begin{array}{l}\text { Date: } \\
8 / 30 / 94\end{array}$ \\
\hline \multicolumn{3}{|c|}{$\begin{array}{l}\text { Assigned To: } \\
\text { SA\&E/PDI (E.P. DiVincenzo/G.A. Tarcza) }\end{array}$} & CIN: \\
\hline \multicolumn{3}{|c|}{ Program WBS Designator: } & $\begin{array}{l}\text { Due Date: } \\
7 / 28 / 95\end{array}$ \\
\hline \multicolumn{3}{|c|}{ Contral Number: SAE-95-006 } & Rev: 0 \\
\hline $\begin{array}{l}\text { MILESTONE TYPE: } \\
\square \text { DOE-HQ } \\
\square \text { DOE-RL } \\
\text { CNTR }\end{array}$ & $\begin{array}{ll} & \text { DIVISION: } \\
& \text { State } \\
\square & \text { Federal } \\
\square & \text { DOE } \\
\square & \text { RCRA } \\
\square & \text { TPA NUMBER }\end{array}$ & $\begin{array}{l}\text { DELIVERABLE: } \\
\square \text { Report } \\
\square \text { Letter } \\
\square \text { Drawings } \\
\text { Other (specify) } \\
\text { ADPE Statement } \\
\text { of Strategy }\end{array}$ & $\begin{array}{l}\text { ADDRESS TO: } \\
\square \text { DOE-HQ } \\
\text { DOE-RL } \\
\text { Other (specify) } \\
\text { BCSR }\end{array}$ \\
\hline
\end{tabular}

Milestone Description:

Develop Safety Analysis \& Engineering FY 1996 Automated Data Processing Equipment and Statement of Strategy.

Description of what constitutes completion of this milestone:

Submit the Safety Analysis \& Engineering FY 1996 Automated Data Processing Equipment and Statement of Strategy to BCS Richland, Inc.

Copies of completed milestone deliverables will be submitted to SA\&E Program Development and Implementation (80170) manager.

\begin{tabular}{|ll|l|}
\hline $\begin{array}{l}\text { Cost Account Manager } \\
\text { E.P. DiVincenzo }\end{array}$ & Date & P.P. DiVincenzolProject Manager \\
\hline $\begin{array}{l}\text { Program Element Manager } \\
\text { E.P. DiVincenzo }\end{array}$ & Date & DOE Monitor \\
\hline
\end{tabular}


WHC-SP-1116 REV 0

W.B $\begin{aligned} & \text { Westinghouse Hanford Company } \\ & \text { MILESTONE DESCRIPTION SHEET }\end{aligned}$
,

\begin{tabular}{|c|c|c|c|c|}
\hline \multicolumn{3}{|c|}{$\begin{array}{l}\text { Title: } \\
\text { Safety Analysis \& Engineering Site Management System Report }\end{array}$} & \multicolumn{2}{|c|}{$\begin{array}{l}\text { Date: } \\
8 / 30 / 94\end{array}$} \\
\hline \multicolumn{3}{|c|}{$\begin{array}{l}\text { Assigned To: } \\
\text { SA\&E/PDI (E.P. DiVincenzo/G.A. Tarcza) }\end{array}$} & \multicolumn{2}{|l|}{ CIN: } \\
\hline \multicolumn{3}{|c|}{$\begin{array}{l}\text { Program WBS Designator: } \\
6.3 .5 .2 .1 \text { (TPCN\# J8DIE) }\end{array}$} & \multicolumn{2}{|c|}{$\begin{array}{l}\text { Due Date: } \\
9 / 15 / 95\end{array}$} \\
\hline \multicolumn{3}{|c|}{ Control Number: SAE-95-008 } & Rev: & \\
\hline $\begin{array}{l}\text { MILESTONE TYPE: } \\
\square \text { DOE-HQ } \\
\square \text { DOE-RL } \\
\text { CNTR }\end{array}$ & $\begin{array}{ll} & \text { DIVISION: } \\
\square & \text { State } \\
\square & \text { Federal } \\
\square & \text { DOE } \\
\square & \text { RCRA } \\
\square & \text { TPA NUMBER }\end{array}$ & $\begin{array}{l}\text { DELIVERABLE: } \\
\text { Report } \\
\square \text { Letter } \\
\square \text { Drawings } \\
\square \text { Other (specify) }\end{array}$ & $\begin{array}{l}\square \\
\square \\
\square\end{array}$ & $\begin{array}{l}\text { ADDRESS TO: } \\
\text { DOE-HQ } \\
\text { DOE-RL } \\
\text { Other (specify) } \\
\text { WHC Performance } \\
\text { Reporting }\end{array}$ \\
\hline
\end{tabular}

Milestone Description:

Prepare monthiy Safety Analysis \& Engineering Site Management System Report.

Description of what constitutes completion of this milestone:

Issue the Safety Analysis \& Engineering Site Management System report to WHC Performance Reporting monthly.

Copies of completed milestone deliverables will be submitted to SA\&E Program Development and Implementation (80170) manager.

\begin{tabular}{|ll|l|}
\hline $\begin{array}{l}\text { Cost Account Manager } \\
\text { E.P. DiVincenzo }\end{array}$ & Date & Program/Project Manager \\
\hline $\begin{array}{l}\text { Program Element Manager } \\
\text { E.P. DiVincenzo }\end{array}$ & Date & DOE Monitor
\end{tabular}


3. B

Westinghouse Hanford Company MILESTONE DESCRIPTION SHEET

\begin{tabular}{|c|c|c|c|}
\hline \multicolumn{3}{|c|}{$\begin{array}{l}\text { Title: } \\
\text { Safety Analysis \& Engineering Variance and Corrective Action Report }\end{array}$} & $\begin{array}{l}\text { Date: } \\
8 / 30 / 94\end{array}$ \\
\hline \multicolumn{3}{|c|}{$\begin{array}{l}\text { Assigned To: } \\
\text { SA\&E/PDI (E.P. DiVincenzo/S.M. Godfrey) }\end{array}$} & CIN: \\
\hline \multicolumn{3}{|c|}{ Program WBS Designator: } & $\begin{array}{l}\text { Due Date: } \\
9 / 15 / 95\end{array}$ \\
\hline \multicolumn{3}{|c|}{ Control Number: SAE-95-009 } & Rev: 0 \\
\hline $\begin{array}{l}\text { MILESTONE TYPE: } \\
\square \text { DOE-HQ } \\
\square \text { DOE-RL } \\
\text { CNTR }\end{array}$ & $\begin{array}{ll} & \text { DIVISION: } \\
& \\
\square & \text { State } \\
\square & \text { Federal } \\
\square & \text { DOE } \\
\square & \text { RCRA } \\
\square & \text { TPA NUMBER }\end{array}$ & $\begin{array}{ll} & \text { DELIVERABLE: } \\
& \text { Report } \\
\square & \text { Letter } \\
\square & \text { Drawings } \\
\square & \text { Other (specify) }\end{array}$ & $\begin{array}{l}\text { ADDRESS TO: } \\
\square \text { DOE-HQ } \\
\text { DOE-RL } \\
\square \text { Other (specify) }\end{array}$ \\
\hline
\end{tabular}

Milestone Description:

Prepare monthly cost variance and analysis report for cost Account P1an 1J8D10.

Description of what constitutes completion of this milestone:

Issue monthiy variance and corrective action reports to DOE-RL at monthly Program Review. Copies of completed milestone deliverables will be submitted to SA\&E Program Development and Implementation (8D170) manager.

Cost Account Mañager

E.P. DiVincenzo

Program Element Manager

E.P. DiVincenzo
Date

Date

DOE Monitor

DOE Monitor
Program/Project Manager 5 Date

E.P. Divincenzo

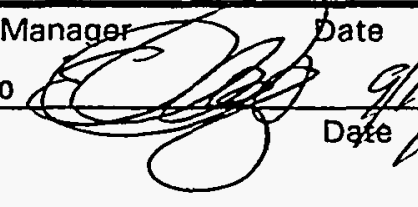


WHC-SP-1116 REV 0

3.B

Westinghouse Hanford Company MILESTONE DESCRIPTION SHEET

\begin{tabular}{|c|c|c|c|}
\hline \multicolumn{3}{|c|}{$\begin{array}{l}\text { Title: Safety Analysis \& Engineering 1J8D10 Program Development and } \\
\text { Implementation Program Review }\end{array}$} & $\begin{array}{l}\text { Date: } \\
8 / 30 / 94\end{array}$ \\
\hline \multicolumn{3}{|c|}{$\begin{array}{l}\text { Assigned To: } \\
\text { SA\&E/PDI (E.P. DiVincenzo) }\end{array}$} & CIN: \\
\hline \multicolumn{3}{|c|}{$\begin{array}{l}\text { Program WBS Designator: } \\
6.3 .5 .2 .1 \text { (TPCN\# J8D1E) }\end{array}$} & $\begin{array}{l}\text { Due Date: } \\
9 / 29 / 95\end{array}$ \\
\hline \multicolumn{3}{|c|}{ Control Number: SAE-95-010 } & Rev: 0 \\
\hline $\begin{array}{l}\text { MILESTONE TYPE: } \\
\square \text { DOE-HQ } \\
\square \text { DOE-RL } \\
\text { CNTR }\end{array}$ & \begin{tabular}{ll} 
& DIVISION: \\
& \multicolumn{1}{c}{} \\
$\square$ & State \\
$\square$ & Federal \\
$\square$ & DOE \\
$\square$ & RCRA \\
$\square$ & TPA NUMBER
\end{tabular} & $\begin{array}{ll} & \text { DELIVERABLE: } \\
\square & \text { Report } \\
\square & \text { Letter } \\
\square & \text { Drawings } \\
\text { Other (specify) } \\
\text { Program Review }\end{array}$ & 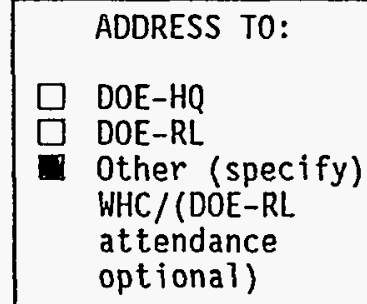 \\
\hline
\end{tabular}

Milestone Description:

Prepare program review presentation materials monthly for Safety Analysis \& Engineering $1 \mathrm{~J} 8010$.

Description of what constitutes completion of this milestone:

Conduct monthly Safety Analysis \& Engineering program reviews. Document with meeting minutes.

Copies of completed milestone deliverables will be submitted to SA\&E Program Development and Implementation (80170) manager.

\begin{tabular}{|l|l|}
$\begin{array}{l}\text { Cost Account Manager } \\
\text { E.P. DiVincenzo }\end{array}$ & $\begin{array}{l}\text { Program/Project Manager } \\
\text { E.P. DiVincenzo }\end{array}$ \\
$\begin{array}{l}\text { Program Element Manager } \\
\text { E.P. DiVincenzo }\end{array}$ & DOE Monitor
\end{tabular}

Overheads, Safety Analysis \& Engineering Page 3-14 
WHC-SP-1116 REV 0

\begin{tabular}{|c|c|c|c|}
\hline 3. B & \multicolumn{2}{|c|}{$\begin{array}{l}\text { Westinghouse Hanford Company } \\
\text { MILESTONE DESCRIPTION SHEET }\end{array}$} & \\
\hline \multicolumn{3}{|c|}{$\begin{array}{l}\text { Title: Quarterly Progress Report of Safety Analysis \& Engineering } \\
\text { Program }\end{array}$} & $\begin{array}{l}\text { Date: } \\
8 / 30 / 94\end{array}$ \\
\hline \multicolumn{3}{|c|}{$\begin{array}{l}\text { Assigned To: } \\
\text { SA\&E/PDI (E.P. DiVincenzo/S.M. Godfrey/G.A. Tarcza) }\end{array}$} & CIN: \\
\hline \multicolumn{3}{|c|}{ Program WBS Designator: } & $\begin{array}{l}\text { Due Date: } \\
1 / 16 / 95\end{array}$ \\
\hline \multicolumn{3}{|c|}{ Control Number: SAE-95-011 } & Rev: 0 \\
\hline $\begin{array}{l}\text { MILESTONE TYPE: } \\
\square \text { DOE-HQ } \\
\text { DOE-RL } \\
\square \text { CNTR }\end{array}$ & $\begin{array}{ll} & \text { DIVISION: } \\
\square & \text { State } \\
\square & \text { Federal } \\
\text { DOE } \\
\square \text { RCRA } \\
\square \text { TPA NUMBER }\end{array}$ & $\begin{array}{ll} & \text { DELIVERABLE: } \\
\square & \text { Report } \\
\square & \text { Letter } \\
\square & \text { Drawings } \\
\text { Other (specify) } \\
\text { Schedule }\end{array}$ & $\begin{array}{l}\text { ADDRESS TO: } \\
\text { DOE-HQ } \\
\text { DOE-RL } \\
\text { Other (specify) }\end{array}$ \\
\hline
\end{tabular}

Milestone Description:

(Milestones SAE-95-007, $-008,-009$, and -010 roll up to support this milestone.)

Prepare and update Safety Analysis \& Engineering Program Progress Report.

Description of what constitutes completion of this milestone:

Issue quarterly Progress Report of SA\&E Program to DOE-RL on January 16, 1995. The

Progress Report will include an update of financial status, work accomplishments and site

Support Program PIan master milestone schedule.

Copies of completed milestone deliverables will be submitted to SA\&E Program Development and Implementation (8D170) manager.

\begin{tabular}{|ll|l|}
\hline Cost ACCOUnt Manager & Date & Program/Project Manager \\
E.P. DiVincenzo & & D.P. DiVincenzote Monitor \\
\hline $\begin{array}{l}\text { Program Element Manager } \\
\text { E.P. DiVincenzo }\end{array}$ & Date & M.R. Moreno
\end{tabular}


WHC-SP-1116 REV 0

\begin{tabular}{|c|c|c|c|}
\hline \multicolumn{4}{|c|}{$\begin{array}{l}\text { Westinghouse Hanford Company } \\
\text { MILESTONE DESCRIPTION SHEET }\end{array}$} \\
\hline \multicolumn{3}{|c|}{$\begin{array}{l}\text { Title: Quarterly Progress Report of Safety Analys is \& Engineering } \\
\text { Program }\end{array}$} & $\begin{array}{l}\text { Date: } \\
8 / 30 / 94\end{array}$ \\
\hline \multicolumn{3}{|c|}{$\begin{array}{l}\text { Assigned To: } \\
\text { SA\&E/PDI (E.P. DiVincenzo/S.M. Godfrey/G.A. Tarcza) }\end{array}$} & CIN: \\
\hline \multicolumn{3}{|c|}{$\begin{array}{l}\text { Program WBS Designator: } \\
6.3 .5 .2 .1 \text { (TPCN\# J8D1E) }\end{array}$} & $\begin{array}{l}\text { Due Date: } \\
4 / 14 / 95\end{array}$ \\
\hline \multicolumn{3}{|c|}{ Control Number: $\quad$ SAE-95-012 } & Rev: 0 \\
\hline $\begin{array}{l}\text { MILESTONE TYPE: } \\
\square \text { DOE-HQ } \\
\text { DOE-RL } \\
\square \text { CNTR }\end{array}$ & $\begin{array}{ll} & \text { DIVISION: } \\
\square & \text { State } \\
\square & \text { Federal } \\
\text { DOE } \\
\square \text { RCRA } \\
\square \text { TPA NUMBER }\end{array}$ & $\begin{array}{ll} & \text { DELIVERABLE: } \\
\square & \text { Report } \\
\square & \text { Letter } \\
\square & \text { Drawings } \\
\text { Other (specify) } \\
\text { Schedule }\end{array}$ & $\begin{array}{ll} & \text { ADDRESS TO: } \\
\square & \text { DOE-HQ } \\
\text { DOE-RL } \\
\text { Other (specify) }\end{array}$ \\
\hline
\end{tabular}

Milestone Description:

(Milestones SAE-95-007, $-008,-009$, and -010 roll up to support this milestone.)

Prepare and update Safety Analysis' \& Engineering Program Progress Report.

Description of what constitutes completion of this milestone:

Issue quarterly Progress Report of SA\&E Program to DOE-RL on Apri1 14, 1995. The Progress Report will include an update of financial status, work accomplishments and Site Support Program Plan master milestone schedule.

Copies of completed milestone deliverables will be submitted to SA\&E Program Development and Implementation (8D170) manager.

\begin{tabular}{|c|c|c|}
\hline $\begin{array}{l}\text { Cost Account Manager } \\
\text { E.P. DiVincenzo }\end{array}$ & Date & $\begin{array}{l}\text { Program/Project Manage } \\
\text { E.P. DiVincenzo }\end{array}$ \\
\hline $\begin{array}{l}\text { Program Element Manager } \\
\text { E.P. DiVincenzo }\end{array}$ & Date & M.R. Moreno Monitor \\
\hline
\end{tabular}


WHC-SP-1116 REV 0

Westinghouse Hanford Company
MILESTONE DESCRIPTION SHEET

Title: Quarterly Progress Report of Safety Analysis \& Engineering Program

Assigned To:

SA\&E/PDI (E.P. DiVincenzo/S.M. Godfrey/G.A. Tarcza)

Program WBS Designator:

6.3.5.2.1 (TPCN\# J8D1E)

Control Number: SAE-95-013

\begin{tabular}{|c|c|c|c|}
\hline $\begin{aligned} \text { MILESTONE TYPE: } \\
\square \text { DOE-HQ } \\
\text { DOE-RL } \\
\square \text { CNTR }\end{aligned}$ & $\begin{aligned} & \text { DIVISION: } \\
& \square \text { State } \\
& \square \text { Federal } \\
& \text { DOE } \\
& \square \text { RCRA } \\
& \square \text { TPA NUMBER }\end{aligned}$ & $\begin{array}{l}\text { DELIVERABLE: } \\
\square \text { Report } \\
\square \text { Letter } \\
\square \text { Drawings } \\
\square \text { Other (specify) } \\
\text { Schedute }\end{array}$ & $\begin{array}{ll} & \text { ADDRESS TO: } \\
\square & \text { DOE-HQ } \\
\text { DOE-RL } \\
\square \text { Other (specify) }\end{array}$ \\
\hline
\end{tabular}

Milestone Description:

(Milestones SAE-95-001, $-005,-007,-008,-009$, and -010 roll up to support this milestone.)

Prepare and update Safety Analysis \& Engineering Program Progress Report. .

Description of what constitutes completion of this milestone:

Issue quarterly Progress Report of SA\&E Program to DOE-RL on July 14, 1995. The Progress Report will include an update of financial status, work accomplishments and Site Support

Program Plan master milestone schedule.

Copies of completed milestone deliverables will be submitted to SA\&E Program Development and Implementation (8D170) manager.

\begin{tabular}{|c|c|c|c|}
\hline $\begin{array}{l}\text { Cost Account Manager } \\
\text { E.P. DiVincenzo }\end{array}$ & Date & $\begin{array}{l}\text { Program/Project } \\
\text { E.P. DiVincenzo }\end{array}$ & $\begin{array}{l}\text { Date } \\
\text { glaolact }\end{array}$ \\
\hline $\begin{array}{l}\text { Program Element Manager } \\
\text { E.P. DiVincenzo }\end{array}$ & Date & $\begin{array}{l}\text { DOE Monitor } \\
\text { M.R. Moreno }\end{array}$ & $9 / 20 / 94$ \\
\hline
\end{tabular}


WHC-SP-1116 REV 0

Westinghouse Hanford Company
MILESTONE DESCRIPTION SHEET

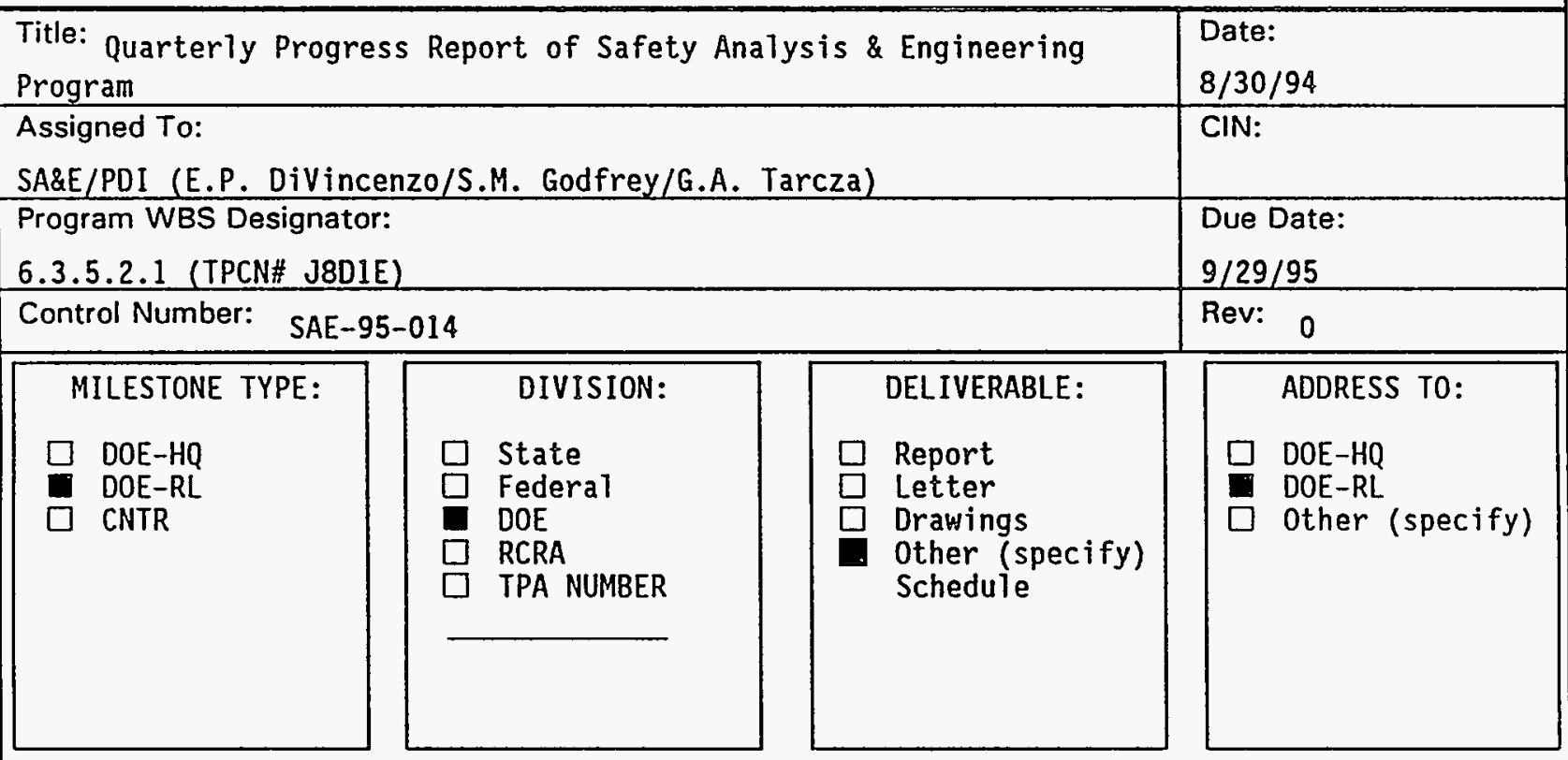

Milestone Description:

(Milestones SAE-95-002, -006, $-007,-008,-009,-010$, and -015 roll up to support this milestone.)

Prepare and update Safety Analys is \& Engineering Program Progress Report.

Description of what constitutes completion of this milestone:

Issue quarterly Progress Report of SA\&E Program to DOE-RL on September 29, 1995. The Progress Report will include an update of financial status, work accomplishments and Site Support Program Plan master milestone schedule.

Copies of completed milestone deliverables will be submitted to SA\&E Program Development and Implementation (80170) manager.

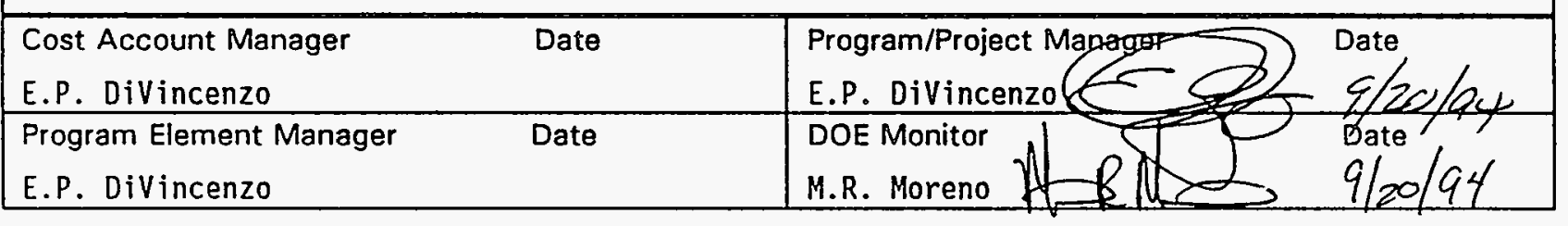


WHC-SP-1116 REV 0

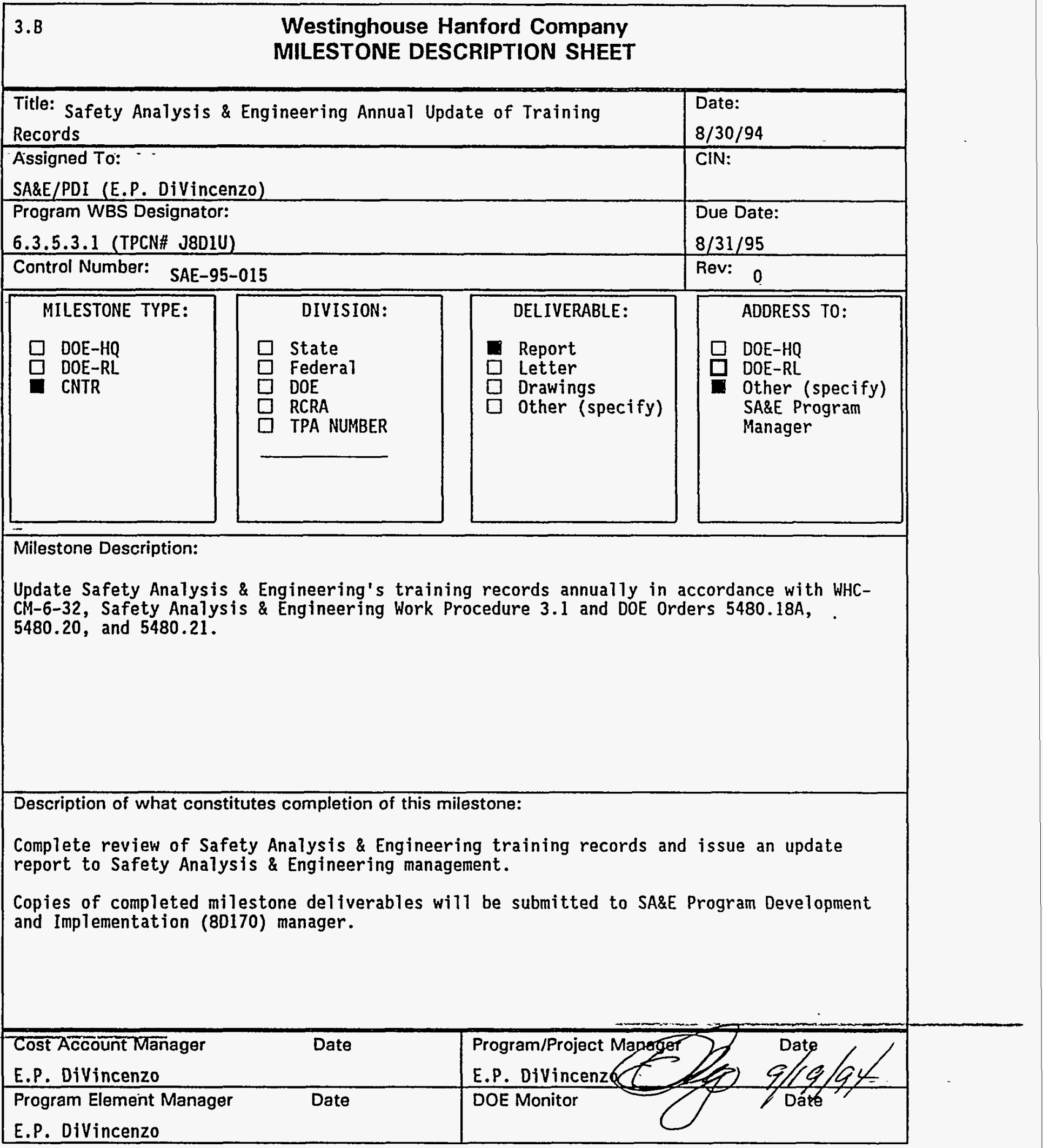


WHC-SP-1116 REV 0

Westinghouse Hanford Company
MILESTONE DESCRIPTION SHEET

\begin{tabular}{|c|c|c|c|}
\hline \multicolumn{3}{|c|}{$\begin{array}{l}\text { Title: } \\
\text { Monthly Status Report of Subcontractor Support }\end{array}$} & $\begin{array}{l}\text { Date: } \\
8 / 30 / 94\end{array}$ \\
\hline \multicolumn{3}{|c|}{$\begin{array}{l}\text { Assigned To: } \\
\text { SA\&E/PDI (E.P. DiVincenzo/D.A. Raap) }\end{array}$} & CIN: \\
\hline \multicolumn{3}{|c|}{$\begin{array}{l}\text { Program WBS Designator: } \\
6.3 .5 .2 .4 \text { (TPCN\# J8D1J) }\end{array}$} & $\begin{array}{l}\text { Due Date: } \\
9 / 29 / 95\end{array}$ \\
\hline \multicolumn{3}{|c|}{ Control Number: SAE-95-016 } & Rev: 0 \\
\hline $\begin{array}{l}\text { MILESTONE TYPE: } \\
\square \text { DOE-HQ } \\
\square \text { DOE-RL } \\
\text { CNTR }\end{array}$ & \begin{tabular}{ll} 
& DIVISION: \\
& \multicolumn{1}{c}{ State } \\
$\square$ & Federal \\
$\square$ & DOE \\
$\square$ & RCRA \\
$\square$ & TPA NUMBER
\end{tabular} & $\begin{array}{l}\text { DELIVERABLE: } \\
\square \text { Report } \\
\square \text { Letter } \\
\square \text { Drawings } \\
\square \text { Other (specify) }\end{array}$ & $\begin{array}{l}\text { ADDRESS TO: } \\
\\
\square \text { DOE-HQ } \\
\text { DOE-RL } \\
\text { Other (specify) } \\
\text { SA\&E Program } \\
\text { Manager }\end{array}$ \\
\hline
\end{tabular}

Milestone Description:

Produce monthly status report of subcontractor support to include update of the outsourcing database.

Description of what constitutes completion of this milestone:

Submit monthly report to SA\&E Program Manager.

Copies of completed milestone deliverables will be submitted to SA\&E Program Development and Implementation (8D170) manager.

\begin{tabular}{|c|c|c|c|}
\hline $\begin{array}{l}\text { Cost Account Manager } \\
\text { E.P. DiVincenzo }\end{array}$ & Date & $\begin{array}{l}\text { Program/Project Managep } \\
\text { E.P. DiVincenzo }\end{array}$ & $5 / 19 / 44$ \\
\hline $\begin{array}{l}\text { Program Element Manager } \\
\text { E.P. DiVincenzo }\end{array}$ & Date & $\begin{array}{l}\text { DOE Monitor } \\
\text { M.R. Moreno }\end{array}$ & \\
\hline
\end{tabular}


WHC-SP-1116 REV 0

\section{B \\ Westinghouse Hanford Company MILESTONE DESCRIPTION SHEET}

\begin{tabular}{|c|c|c|c|}
\hline \multicolumn{3}{|c|}{$\begin{array}{l}\text { Title: } \\
\text { Quarterly Outsourcing Status Report }\end{array}$} & $\begin{array}{l}\text { Date: } \\
8 / 30 / 94 \\
\end{array}$ \\
\hline \multicolumn{3}{|c|}{$\begin{array}{l}\text { Assigned To: } \\
\text { SA\&E/PDI (E.P. DiVincenzo/D.A. Raap) }\end{array}$} & CIN: \\
\hline \multicolumn{3}{|c|}{$\begin{array}{l}\text { Program WBS Designator: } \\
6.3 .5 .2 .4 \text { (TPCN\# J8D1J) }\end{array}$} & $\begin{array}{l}\text { Due Date: } \\
1 / 16 / 95\end{array}$ \\
\hline \multicolumn{3}{|c|}{ Control Number: SAE-95-017 } & Rev: 0 \\
\hline $\begin{array}{l}\text { MILESTONE TYPE: } \\
\square \text { DOE-HQ } \\
\text { DOE-RL } \\
\square \text { CNTR }\end{array}$ & $\begin{array}{ll} & \text { DIVISION: } \\
\square & \text { State } \\
\square & \text { Federal } \\
\text { DOE } \\
\square \text { RCRA } \\
\square \text { TPA NUMBER }\end{array}$ & $\begin{array}{l}\text { DELIVERABLE: } \\
\\
\text { Report } \\
\square \text { Letter } \\
\square \text { Drawings } \\
\square \text { Other (specify) }\end{array}$ & $\begin{array}{l}\text { ADDRESS TO: } \\
\square \text { DOE-HQ } \\
\text { DOE-RL } \\
\text { Other (specify) }\end{array}$ \\
\hline
\end{tabular}

Milestone Description:

(Milestone SAE-95-016 rolls up to support this milestone.)

Produce quarterly outsourcing status report to include status and volume of outsourced technical support.

Description of what constitutes completion of this milestone:

Submit quarterly outsourcing status report to DOE-RL.

Copies of completed milestone deliverables will be submitted to SA\&E Program Development and Implementation (8D170) manager.

\begin{tabular}{|c|c|c|}
\hline $\begin{array}{l}\text { Cost Accoưnt Mañager } \\
\text { E.P. DiVincenzo }\end{array}$ & Date & E.P. DiVincenzates \\
\hline $\begin{array}{l}\text { Program Element Manage } \\
\text { E.P. DiVincenzo }\end{array}$ & Date & $\begin{array}{l}\text { DOE Monitor } \\
\text { M.R. Moreno }\end{array}$ \\
\hline
\end{tabular}


WHC-SP-1116 REV 0

\begin{tabular}{|c|c|c|c|}
\hline \multicolumn{4}{|c|}{$\begin{array}{l}\text { Westinghouse Hanford Company } \\
\text { MILESTONE DESCRIPTION SHEET }\end{array}$} \\
\hline \multicolumn{3}{|c|}{$\begin{array}{l}\text { Title: } \\
\text { Quarteriy Outsourcing Status Report }\end{array}$} & $\begin{array}{l}\text { Date: } \\
8 / 30 / 94\end{array}$ \\
\hline \multicolumn{3}{|c|}{$\begin{array}{l}\text { Assigned To: } \\
\text { SA\&E/PDI (E.P. DiVincenzo/D.A. Raap) }\end{array}$} & CIN: \\
\hline \multicolumn{3}{|c|}{$\begin{array}{l}\text { Program WBS Designator: } \\
6.3 .5 .2 .4 \text { (TPCN\# J801J) }\end{array}$} & $\begin{array}{l}\text { Due Date: } \\
4 / 14 / 95\end{array}$ \\
\hline \multicolumn{3}{|c|}{ Control Number: SAE-95-018 } & Rev: 0 \\
\hline $\begin{array}{l}\text { MILESTONE TYPE: } \\
\square \text { DOE-HQ } \\
\text { DOE-RL } \\
\square \text { CNTR }\end{array}$ & $\begin{array}{ll} & \text { DIVISION: } \\
\square & \text { State } \\
\square & \text { Federal } \\
\text { DOE } \\
\square \text { RCRA } \\
\square \text { TPA NUMBER }\end{array}$ & $\begin{array}{l}\text { DELIVERABLE: } \\
\text { Report } \\
\square \text { Letter } \\
\square \text { Drawings } \\
\square \text { Other (specify) }\end{array}$ & $\begin{array}{ll} & \text { ADDRESS TO: } \\
\square & \text { DOE-HQ } \\
\text { DOE-RL } \\
\square \text { Other (specify) }\end{array}$ \\
\hline
\end{tabular}

Milestone Description:

(Milestone SAE-95-016 rolls up to support this milestone.)

Produce quarterly outsourcing status report to include status and volume of outsourced technical support.

Description of what constitutes completion of this milestone:

Submit quarterly outsourcing status report to DOE-RL.

Copies of completed milestone deliverables will be submitted to SA\&E Program Development and Implementation (8D170) manager.

\begin{tabular}{|ll|l|}
\hline $\begin{array}{l}\text { Cost Account Manager } \\
\text { E.P. DiVincenzo }\end{array}$ & Date & Program/Project Manager \\
\hline $\begin{array}{l}\text { Program Element Manager } \\
\text { E.P. DiVincenzo }\end{array}$ & Date & DOE Monitor \\
M.R. Moreno
\end{tabular}

Overheads, Safety Analysis \& Engineering Page 3-22 
WHC-SP-1116 REV 0

\begin{tabular}{|c|c|c|c|}
\hline $3 . B$ & $\begin{array}{l}\text { Westinghot } \\
\text { MILESTONE }\end{array}$ & $\begin{array}{l}\text { nford Company } \\
\text { RIPTION SHEET }\end{array}$ & \\
\hline $\begin{array}{l}\text { Title: } \\
\text { Quarterly Outsourci }\end{array}$ & atus Report & & $\begin{array}{l}\text { Date: } \\
8 / 30 / 94 \\
\end{array}$ \\
\hline $\begin{array}{l}\text { Assigned To: } \\
\text { SA\&E/PDI (E.P. DiVi) }\end{array}$ & $0 / 0 . A$. Raap) & & CIN: \\
\hline $\begin{array}{l}\text { Program WBS Designa } \\
6.3 .5 .2 .4 \text { (TPCN\# J8 }\end{array}$ & 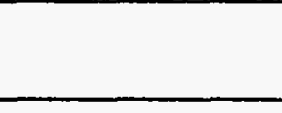 & & $\begin{array}{l}\text { Due Date: } \\
7 / 14 / 95\end{array}$ \\
\hline Control Number: SAE. & & & Rev: $\quad 0$ \\
\hline $\begin{array}{l}\text { MILESTONE TYPE: } \\
\square \text { DOE-HQ } \\
\square \text { DOE-RL } \\
\square \text { CNTR }\end{array}$ & $\begin{aligned} & \text { DIVISION: } \\
& \text { State } \\
& \square \text { Federa1 } \\
& \text { DOE } \\
& \square \\
& \square \text { RCRA } \\
& \square \text { TPA NUMBER }\end{aligned}$ & $\begin{array}{l}\text { DELIVERABLE: } \\
\text { Report } \\
\square \text { Letter } \\
\square \text { Drawings } \\
\square \text { Other (specify) }\end{array}$ & $\begin{array}{l}\text { ADDRESS TO: } \\
\square \text { DOE-HQ } \\
\text { DOE-RL } \\
\square \text { Other (specify) }\end{array}$ \\
\hline $\begin{array}{l}\text { Milestone Description: } \\
\text { (Milestone SAE-95-0 } \\
\text { Produce quarterly o } \\
\text { technical support. }\end{array}$ & $\begin{array}{l}11 \text { s up to suppor } \\
\text { rrcing status rep }\end{array}$ & $\begin{array}{l}\text { milestone.) } \\
\text { include status and vo }\end{array}$ & of outsourced \\
\hline $\begin{array}{l}\text { Description of what co } \\
\text { Submit quarterly ou } \\
\text { Copies of completed } \\
\text { and Implementation }\end{array}$ & $\begin{array}{l}\text { tes completion of } \\
\text { cing status repc } \\
\text { stone deliverabl } \\
\text { 0) manager. }\end{array}$ & $\begin{array}{l}\text { Stone: } \\
\text { DOE-RL. } \\
\text { be submitted to SA }\end{array}$ & gram Development \\
\hline $\begin{array}{l}\text { Cost AcCoúnt Maniage } \\
\text { E.P. DiVincenzo }\end{array}$ & Date & $\begin{array}{l}\text { Program/Project Manage } \\
\text { E.P. DiVincenzo }\end{array}$ & $\sum_{2}$ Date \\
\hline $\begin{array}{l}\text { Program Element Mane } \\
\text { E.P. DiVincenzo }\end{array}$ & Date & M.R. Moreno Monitor & $3 \quad 9 / 20194$ \\
\hline
\end{tabular}

Overhead's, Safety Analysis \& Engineering Page 3-23 
WHC-SP-1116 REV 0

3.B Westinghouse Hanford Company
MILESTONE DESCRIPTION SHEET

\begin{tabular}{|c|c|c|c|}
\hline \multicolumn{3}{|c|}{$\begin{array}{l}\text { Title: } \\
\text { Quarterly Outsourcing Status Report }\end{array}$} & $\begin{array}{l}\text { Date: } \\
8 / 30 / 94\end{array}$ \\
\hline \multicolumn{3}{|c|}{$\begin{array}{l}\text { Assigned To: } \\
\text { SA\&E/PDI (E.P. DiVincenzo/D.A. Raap) }\end{array}$} & CIN: \\
\hline \multicolumn{3}{|c|}{$\begin{array}{l}\text { Program W8S Designator: } \\
6.3 .5 .2 .4 \text { (TPCN\# J8D1J) }\end{array}$} & $\begin{array}{l}\text { Due Date: } \\
9 / 29 / 95\end{array}$ \\
\hline \multicolumn{3}{|c|}{ Control Number: SAE-95-020 } & Rev: 0 \\
\hline $\begin{array}{l}\text { MILESTONE TYPE: } \\
\square \text { DOE-HQ } \\
\text { DOE-RL } \\
\square \text { CNTR }\end{array}$ & $\begin{aligned} & \text { DIVISION: } \\
& \square \text { State } \\
& \square \text { Federal } \\
& \text { DOE } \\
& \square \\
& \text { RCRA } \\
& \square \text { TPA NUMBER }\end{aligned}$ & $\begin{array}{l}\text { DELIVERABLE: } \\
\text { Report } \\
\square \text { Letter } \\
\square \text { Drawings } \\
\square \text { Other (specify) }\end{array}$ & $\begin{array}{l}\text { ADDRESS TO: } \\
\square \text { DOE-HQ } \\
\text { DOE-RL } \\
\square \text { Other (specify) }\end{array}$ \\
\hline
\end{tabular}

Milestone Description:

(Milestone SAE-95-016 rolls up to support this milestone.)

Produce quarterly outsourcing status report to include status and volume of outsourced technical support.

Description of what constitutes completion of this milestone:

Submit quarterly outsourcing status report to DOE-RL.

Copies of completed milestone deliverables will be submitted to SA\&E Program Development and Implementation (80170) manager.

\begin{tabular}{|c|c|c|}
\hline $\begin{array}{l}\text { Cost Account Manager } \\
\text { E.P. DiVincenzo }\end{array}$ & Date & Program/Project Manages DiVincenzos S/20/aL \\
\hline $\begin{array}{l}\text { Program Element Manager } \\
\text { E.P. DiVincenzo }\end{array}$ & Date & M.R. Moreno \\
\hline
\end{tabular}


WHC-SP-1116 REV 0

\begin{tabular}{|c|c|c|c|}
\hline \multicolumn{4}{|c|}{$\begin{array}{l}\text { Westinghouse Hanford Company } \\
\text { MILESTONE DESCRIPTION SHEET }\end{array}$} \\
\hline \multicolumn{3}{|c|}{$\begin{array}{l}\text { Title: } \\
\text { Draft Outline of Safety Analysis and Engineering Transition Plan }\end{array}$} & $\begin{array}{l}\text { Date: } \\
8 / 30 / 94\end{array}$ \\
\hline \multicolumn{3}{|c|}{$\begin{array}{l}\text { Assigned To:-- } \\
\text { SA\&E (Management) }\end{array}$} & CIN: \\
\hline \multicolumn{3}{|c|}{ Program WBS Designator: } & $\begin{array}{l}\text { Due Date: } \\
11 / 15 / 95\end{array}$ \\
\hline \multicolumn{3}{|c|}{ Control Number: SAE-95-021 } & Rev: 0 \\
\hline $\begin{array}{l}\text { MILESTONE TYPE: } \\
\square \text { DOE-HQ } \\
\square \text { DOE-RL } \\
\text { CNTR }\end{array}$ & $\begin{array}{l}\text { DIVISION: } \\
\text { State } \\
\square \text { Federar } \\
\square \text { DOE } \\
\square \text { RCRA } \\
\square \text { TPA NUMBER }\end{array}$ & 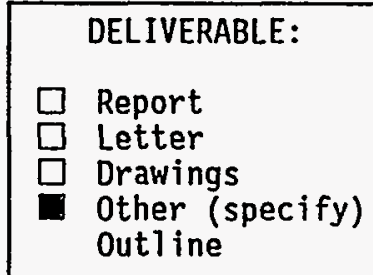 & $\begin{array}{l}\text { ADDRESS TO: } \\
\square \text { DOE-HQ } \\
\square \text { DOE-RL } \\
\text { Other (specify) } \\
\text { EAT Management }\end{array}$ \\
\hline \multicolumn{4}{|c|}{$\begin{array}{l}\text { Milestone Description: } \\
\text { Produce the draft outline of the Safety Analysis and Engineering Transition Plan. Through } \\
\text { negotiations with Westinghouse Hanford Company management and DOE-RL, develop planning } \\
\text { packages to include an outline for the transition plan. }\end{array}$} \\
\hline \multicolumn{4}{|c|}{$\begin{array}{l}\text { Description of what constitutes completion of this milestone: } \\
\text { Issue the draft outline of the SA\&E Transition Plan to EAT management. The outline will } \\
\text { include the Westinghouse management and DOE-RL agreed approach for development of the } \\
\text { Safety Analysis and Engineering Transition Plan. } \\
\text { Copies of completed milestone deliverables will be submitted to SA\&E Program Development } \\
\text { and Implementation ( } 80170) \text { manager. }\end{array}$} \\
\hline $\begin{array}{l}\text { Cost Account Manager } \\
\text { E.P. DiVincenzo }\end{array}$ & Date & $\begin{array}{l}\text { Program/Project Manag } \\
\text { E.P. DiVincenze }\end{array}$ & Bt $91 / 9 / 94$ \\
\hline $\begin{array}{l}\text { Program Element Manas } \\
\text { E.P. DiVincenzo }\end{array}$ & Date & $\begin{array}{l}\text { DOE Monitor } \\
\text { M.R. Moreno }\end{array}$ & "Daté \\
\hline
\end{tabular}




\begin{tabular}{|c|c|c|c|}
\hline 3. B & \multicolumn{2}{|c|}{$\begin{array}{l}\text { Westinghouse Hanford Company } \\
\text { MILESTONE DESCRIPTION SHEET }\end{array}$} & \\
\hline \multicolumn{3}{|c|}{$\begin{array}{l}\text { Title: } \\
\text { Safety Analysis and Engineering Transition Plan }\end{array}$} & $\begin{array}{l}\text { Date: } \\
8 / 30 / 94\end{array}$ \\
\hline \multicolumn{3}{|c|}{$\begin{array}{l}\text { Assigned To: } \\
\text { SA\&E (Management) }\end{array}$} & CIN: \\
\hline \multicolumn{3}{|c|}{$\begin{array}{l}\text { Program WBS Designator: } \\
6.3 .5 .2 .1 \text { (TPCN\# J8D1E) }\end{array}$} & $\begin{array}{l}\text { Due Date: } \\
1 / 15 / 95\end{array}$ \\
\hline \multicolumn{3}{|c|}{ Control Number: SAE-95-022 } & Rev: 0 \\
\hline $\begin{array}{l}\text { MILESTONE TYPE: } \\
\square \text { DOE-HQ } \\
\text { DOE-RL } \\
\square \text { CNTR }\end{array}$ & 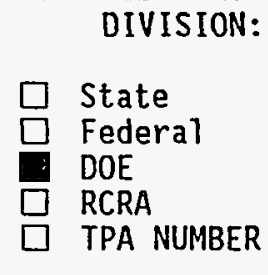 & $\begin{array}{l}\text { DELIVERABLE: } \\
\begin{array}{l}\text { Report } \\
\square \text { Letter } \\
\square \text { Drawings } \\
\text { Other (specify) } \\
\text { Plan }\end{array}\end{array}$ & $\begin{array}{l}\text { ADDRESS TO: } \\
\square \text { DOE-HQ } \\
\text { DOE-RL } \\
\square \text { other (specify) }\end{array}$ \\
\hline \multicolumn{4}{|c|}{$\begin{array}{l}\text { Milestone Description: } \\
\text { (Milestone SAE-95-021 rolls up to support this milestone.) } \\
\text { Develop a transition plan to become Safety Analysis integrating contractor. }\end{array}$} \\
\hline \multicolumn{4}{|c|}{$\begin{array}{l}\text { Description of what constitutes completion of this milestone: } \\
\text { Issue the Safety Analysis and Engineering Transition Plan to define the role of the Safety } \\
\text { Analysis integrating contractor. The plan will include the approach, plan and schedule to } \\
\text { downsize the department and align the new organization for the new mission as integrating } \\
\text { contractor. } \\
\text { Copies of completed milestone deliverables will be submitted to SA\&E Program Development } \\
\text { and Implementation (80170) manager. }\end{array}$} \\
\hline \multicolumn{2}{|c|}{$\begin{array}{l}\text { Cost Account Manager } \\
\text { E.P. DiVincenzo }\end{array}$} & $\begin{array}{l}\text { Program/Project Mana } \\
\text { E.P. DiVincenzo }\end{array}$ & 72 Date 912019 \\
\hline $\begin{array}{l}\text { Program Element Man } \\
\text { E.P. DiVincenzo }\end{array}$ & Date & DOE Monitor & $\int_{9}^{\text {Date }} 9 / 20 / 94$ \\
\hline
\end{tabular}




\begin{tabular}{|l|c|c|}
\hline 3.C Glossary & $\begin{array}{c}\text { Westinghouse Hanford Company } \\
\text { Overheads, Safety Analysis \& } \\
\text { Engineering, SMS/WBS 6.3.5 }\end{array}$ & $\begin{array}{c}\text { FY 1995 } \\
\text { Site Support Program P1an } \\
\text { Date Prepared: 09/14/94 }\end{array}$ \\
\hline
\end{tabular}

\section{J8010 Safety Analysis \& Engineering Operations}

Safety Analysis \& Engineering Operations is the Cost Account Plan that defines operations management and support functions associated with the development of safety analyses and safety documentation. This element is further subdivided into discrete work packages for the various activities essential for the business unit to function. This element does not include activities associated with development of program- or project-specific safety analyses or safety documentation.

\section{J8D100A Management and Administration}

Management and Administration is the work package that encompasses the management and administrative activities associated with the operations of the SA\&E department and the management of department resources. This element includes task packages for fixed departmental costs, discretionary functional management and administration activities, and provision for hourly support personnel (e.g., 1000-hour personnel).

\section{J8D100A00 Nondiscretionary Operating Costs}

Nondiscretionary Operating Costs provides resources for occupancy charges, Hanford Environmental Health Foundation, management proration, Site services, telephones, and network maintenance.

\section{J8D100A01 Management Activities}

Management Activities provides management, administrative labor, and office supplies for the department. Labor charges are limited to those activities deemed strictly department management business. Support for BCSR computer maintenance services is provided as we 11 .

1J80100A02 Hourly Support

Hourly Support provides for hourly support (retirees) resources for the department.

\section{J8D100A03 Staff Moves}

Staff Moves is the task package that accomplishes office moves and provides office furniture on Site. Funding is also provided for employment candidates' interview travel. Relocation of new hires should be provided by the directfunding program benefitting from the new hire. 
WHC-SP-1116 REV 0

\begin{tabular}{|l|c|c|}
\hline 3.C Glossary & $\begin{array}{c}\text { Westinghouse Hanford Company } \\
\text { Overheads, Safety AnaTysis \& } \\
\text { Engineering, SMS/WBS 6.3.5 }\end{array}$ & $\begin{array}{c}\text { FY 1995 } \\
\text { Site Support Program Plan } \\
\text { Date Prepared: 09/14/94 }\end{array}$ \\
\hline
\end{tabular}

\section{J8D100B Program Management}

This work package provides the resources to accomplish the planning and business management objectives of the department, support the excellence programs, provide for professional society activities, and maintain the required reporting systems.

\section{J8D100B00 Program Development and Implementation}

Detiverables produced by this task package include input to the Site Support Program Plan, Fiscal Year Work Plan, and the Environment, Safety, and Health Management Plan. Also included is fiscal-year overhead-budget development and management, mid-year budget reviews, departmental transition planning, and automated data-processing equipment management.

1J8D100B02 EFCOG

EFCOG provides for participation in DOE Energy Facilities Contractor Operating Groups. It provides for travel, living, and associated expenses while coordinating with other DOE operating contractors and with DOE-sponsored working groups and committees.

1J8D100B03 Professional Societies and Standards Groups

This task package provides for personnel time, travel, and living costs while SA\&E personnel are participating in professional societies and specific standards groups.

IJ8D100B04 Contracts Administration

This task package provides the SA\&E structure for administration and execution of SA\&E contracted-service support.

1J8D100B05 CAM Administration

CAM Administration provides support for the centralized development, implementation, and administration of the Corrective Action Management program within SA\&E. 
WHC-SP-1116 REV 0

\begin{tabular}{|c|c|c|c|}
\hline 3.C Glossary & $\begin{array}{c}\text { Westinghouse Hanford Company } \\
\text { Overheads, Safety Analysis \& } \\
\text { Engineering, SMS/WBS 6.3.5 }\end{array}$ & $\begin{array}{c}\text { Site Support Program PTan } \\
\text { Date Prepared: 09/14/94 }\end{array}$ \\
\hline
\end{tabular}

\section{J8D100E Qualification and Training}

This work package provides for all mandatory and technical training necessary to assure that SA\&E personnel meet the requirements of the various governing policies and procedures within WHC and of DOE orders.

\section{J8D100E00 Mandatory Training}

This task package provides for all the required training of SA\&E personnel to assure compliance with applicable WHC policies and procedures.

\section{J8D100E01-08 Technical Training}

These task packages provide the resources for all onsite and offsite training of SA\&E personnel to achieve and maintain the technical expertise necessary to fulfill the requirements of the SA\&E charter and the Engineering, Analysis and Technology Excellence Program. Tuition reimbursements are included in these task packages.

\section{J8D100G Emergent Work}

This work package provides resources for work imposed on SA\&E that was not planned or budgeted for and that cannot be absorbed into the existing work scope and budget.

1J8D100G00 Emergent Work

The definition for this task package is the same as that for 1J8D100G. 
WHC-SP-1116 REV 0

\begin{tabular}{|c|c|c|c|c|}
\hline \multicolumn{2}{|c|}{$\begin{array}{l}\text { 3.D FY } 1995 \text { Task } \\
\text { Package Control } \\
\text { Number Index }\end{array}$} & \multicolumn{2}{|c|}{$\begin{array}{c}\text { Westinghouse Hanford Company } \\
\text { Overheads, Safety Analys is \& } \\
\text { Engineering, SMS/WBS } 6.3 .5\end{array}$} & \multirow{2}{*}{$\begin{array}{l}\text { FY } 1995 \\
\text { Site Support Program Plan } \\
\text { Date Prepared: } 09 / 14 / 94 \\
\text { Task package }\end{array}$} \\
\hline WBS & TPCN & Manager & & \\
\hline $1 \mathrm{J8D10}$ & & E. P. DiVincenzo & $\begin{array}{l}\text { Safet } \\
\text { Opera }\end{array}$ & $\begin{array}{l}\text { Analysis \& Engineering } \\
\text { ions }\end{array}$ \\
\hline $1 \mathrm{~J} 8 \mathrm{D} 100 \mathrm{~A}$ & & E. P. DiVincenzo & Manag & ment and Administration \\
\hline $1 J 80100 A 00$ & J8D1A & E. P. DiVincenzo & Nondi & cretionary Operating Costs \\
\hline $1 J 80100 A 01$ & J8D1B & E. P. DiVincenzo & 80100 & Management Activities \\
\hline $1 J 80100 A 02$ & J8D1C & E. P. DiVincenzo & Hour $]$ & Support \\
\hline $1 \mathrm{~J} 8 \mathrm{D} 100 \mathrm{A03}$ & J8D1D & E. P. DiVincenzo & Staff & Moves \\
\hline $1 \mathrm{~J} 80100 \mathrm{~B}$ & & & Progr & m Management \\
\hline 1J8D100B00 & J8DIE & E. P. DiVincenzo & $\begin{array}{l}\text { Progr } \\
\text { Imple }\end{array}$ & $\begin{array}{l}m \text { Development and } \\
\text { entation }\end{array}$ \\
\hline $1 \mathrm{~J} 80100 \mathrm{BO} 2$ & J8D1G & J. J. Zimmer & EFCOG & Activities \\
\hline 1J8D100B03 & J8D1H & E. P. DiVincenzo & $\begin{array}{l}\text { Profe } \\
\text { Stand }\end{array}$ & $\begin{array}{l}\text { sional Societies and } \\
\text { rds Groups }\end{array}$ \\
\hline 1J8D100B04 & J8D1J & E. P. DiVincenzo & Contr & cts Administration \\
\hline $1 J 80100 B 05$ & J8DIK & E. P. DiVincenzo & CAM A & ministration \\
\hline $1 \mathrm{~J} 8 \mathrm{D} 100 \mathrm{E}$ & & & Quali & ications and Training \\
\hline $1 \mathrm{~J} 8 \mathrm{D} 100 \mathrm{E00}$ & J8D1U & E. P. DiVincenzo & Manda & ory Training \\
\hline 1J8DIO0E01 & J8D1V & E. P. DiVincenzo & 80100 & Technical Training \\
\hline $1 \mathrm{~J} 80100 \mathrm{E} 02$ & J8D1W & K. 0. Fein & 80110 & Technical Training \\
\hline 1J80100E03 & J8DIY & L. E. Johnson & 80120 & Technical Training \\
\hline $1 J 80100 E 05$ & J8D11 & J. J. Zimmer & $8 D 140$ & Technical Training \\
\hline 1J80100E06 & J8D12 & J. H. Daughtry & $8 D 150$ & Technical Training \\
\hline 1J8D100E08 & J8D14 & E. P. DiVincenzo & $8 D 170$ & Technical Training \\
\hline $1 \mathrm{~J} 80100 \mathrm{G}$ & & & Emerg & nt Work \\
\hline 1J8D100G00 & J8D17 & E. P. DiVincenzo & Emerg & nt Work \\
\hline
\end{tabular}




\section{WHC-SP-1116 REV 0}

FOS-B620-R

CANBS: 1 JEDIO SAFETY AHLLYSIS \& ENGIHEERIKG

CA Manager: OIVIKCarzo, EP

Resp Org: 80100 SAFETY AMULYSIS \& ENGINEERING Budget Analyst: GS - SAMELS, KA

G. Index: 8 \& $R$ unber: NHO100000 B \& R Title: UDISTRI\&IED COSTS

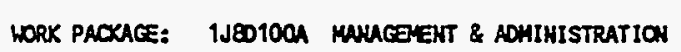

Resp Org:
BD100 SAFETY AHALYSIS \& ENGIKEERING

FIXED OPERATION COSTS
Scope of Work:

\section{Cap Rev: 00 Cin: Start Completion} Budget (KS): 3664.4 Date Date Phone: 376-191110/01/94 09/30/55 CAP STATUS: 0

Revision No: 00 Date: 08/02/94

CAP STATUS: D

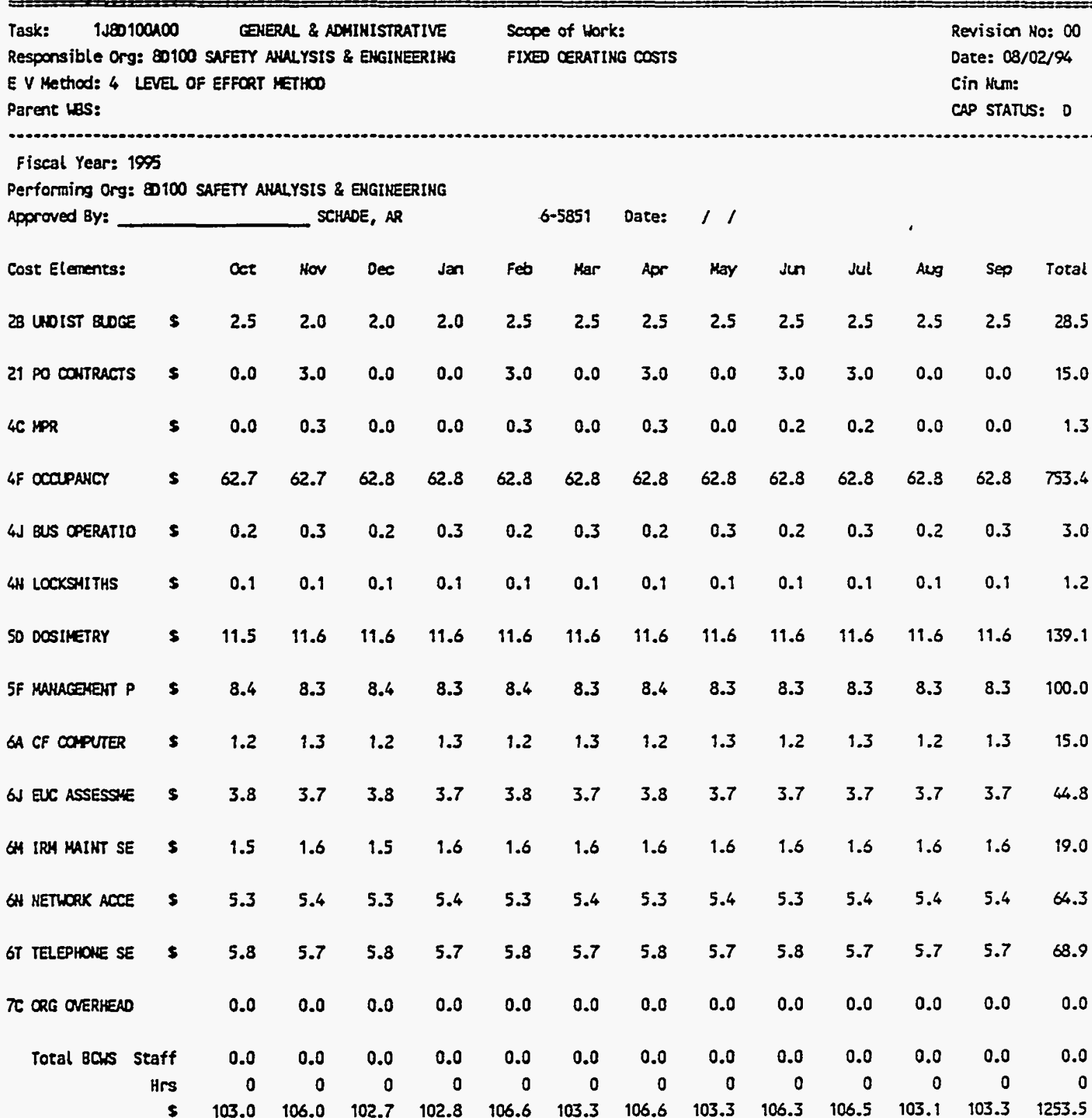

Total 8CP: 
Task: 1J80100A01 CENERAL \& ADMIHISTRATIVE Responsible Org: 80100 SAFETY ANULYSIS \& EMGIHEERIMG E V Method: 4 LEVEL OF EFFCRT VETHCO Parent HB:
Scope of Work:

80100 KoT ACTIVITIES
Revision No: 00 Date: $08 / 02 / 94$ Cin Num: CAP STATUS: 0

Fiscal Year: 1995

Performing Org: 80100 SAFETY ANaLYSIS \& ENGINEERING

Approved By: SCHADE, AR

Cost Elements: oct Hov Dec Jan

$\infty$ hON-EXEPT His Staff $\quad i .0$

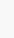
01 EXEPT

$\begin{array}{rll}\text { Staff } & 1.0 & 1.0 \\ \text { Hrs } & 148 & 132 \\ 5 & 6.1 & 5.4\end{array}$

\begin{tabular}{|c|c|c|c|c|c|c|c|c|c|c|c|c|c|c|}
\hline 10 MATL \& EOUIP & s & 0.2 & 0.3 & 0.2 & 0.3 & 0.2 & 0.3 & 0.2 & 0.3 & 0.2 & 0.3 & 0.2 & 0.3 & 3.0 \\
\hline 14 OFFICE SAPPL & s & 0.1 & 0.1 & 0.1 & 0.1 & 0.1 & 0.1 & 0.1 & 0.1 & 0.1 & 0.1 & 0.1 & 0.1 & 1.2 \\
\hline$\approx X$ LOCAL REGIST & $\$$ & 0.1 & 0.1 & 0.1 & 0.1 & 0.9 & 0.1 & 0.1 & 0.1 & 0.1 & 0.1 & 0.1 & 0.1 & 1.2 \\
\hline $4 C \mathrm{MPR}$ & s & 0.1 & 0.0 & 0.0 & 0.0 & 0.1 & 0.1 & 0.1 & 0.1 & 0.1 & 0.1 & 0.1 & 0.1 & 0.9 \\
\hline GA CF COAPUTER & s & 0.5 & 0.5 & 0.5 & 0.5 & 0.0 & 0.0 & 0.0 & 0.0 & 0.0 & 0.0 & 0.0 & 0.0 & \\
\hline \multicolumn{2}{|l|}{ TC ORG OVERHEAD } & 0.0 & 0.0 & 0.0 & 0.0 & 0.0 & 0.0 & 0.0 & 0.0 & 0.0 & 0.0 & 0.0 & 0.0 & \\
\hline \multirow[t]{3}{*}{ Total BCus } & Staff & 2.0 & 2.0 & 2.0 & 2.0 & 2.0 & 2.0 & 2.0 & 2.0 & 2.0 & 2.0 & 2.0 & 2.0 & \\
\hline & Hrs & 296 & 264 & 272 & 326 & 284 & 298 & 366 & 296 & 278 & 312 & 284 & 348 & 3624 \\
\hline & s & 9.7 & 8.8 & 8.9 & 10.6 & 8.8 & 9.6 & 11.3 & 9.3 & 8.7 & 9.8 & 8.8 & 10.8 & \\
\hline
\end{tabular}

Total BCP:

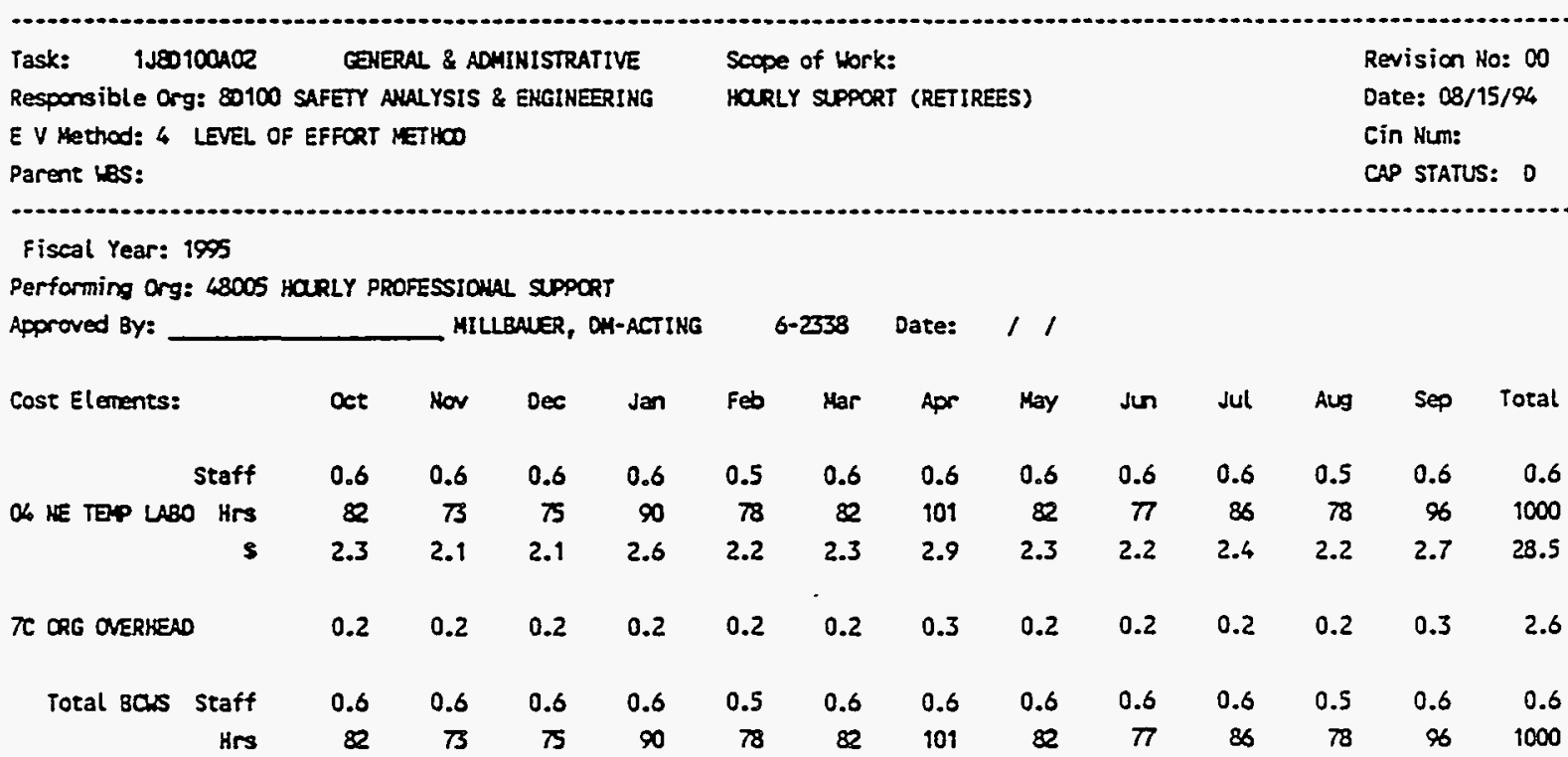




\section{$\begin{array}{llllllllllllll}\text { s } & 2.5 & 2.3 & 2.3 & 2.8 & 2.4 & 2.5 & 3.1 & 2.5 & 2.4 & 2.7 & 2.4 & 3.0 & 31.1\end{array}$}

Total BCP:

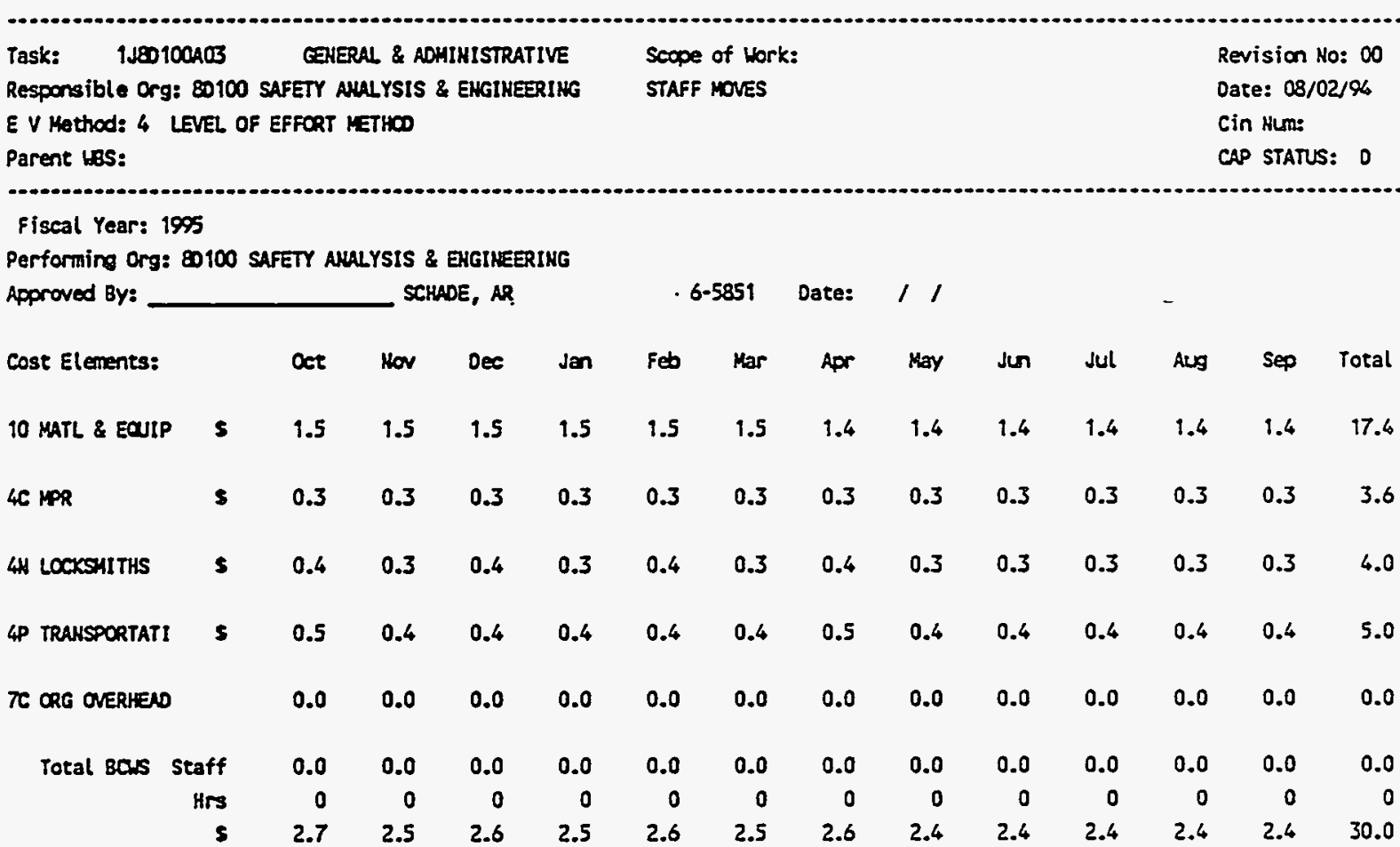

Total BCP:

Total th

oct Nor Dec

$\begin{array}{rrrrr} & \text { Staff } & 1.0 & 1.0 & 1.0 \\ \text { CO NOH-EXEPT } & \text { Hrs } & 148 & 132 & 136 \\ & 5 & 2.6 & 2.3 & 2.4\end{array}$

1.0

1.01 .0

Har Apr

Apr Mar

Jun

In Jul Aug Sep Toral

$\begin{array}{rrrrrrrrrrrrrrr} & \text { Staff } & 1.0 & 1.0 & 1.0 & 1.0 & 1.0 & 1.0 & 1.0 & 1.0 & 1.0 & 1.0 & 1.0 & 1.0 & 1.0 \\ 01 \text { EEEPT } & \text { Hrs } & 148 & 132 & 136 & 163 & 142 & 149 & 183 & 148 & 139 & 156 & 142 & 174 & 1812\end{array}$

$\begin{array}{rcccccccccccccc}01 \text { EEEPT } & \text { Hrs } & 148 & 132 & 136 & 163 & 142 & 149 & 183 & 148 & 139 & 156 & 142 & 174 & 1812 \\ & \$ & 6.1 & 5.4 & 5.6 & 6.7 & 5.8 & 6.1 & 7.5 & 6.1 & 5.7 & 6.4 & 5.8 & 7.1 & 74.6\end{array}$

$\begin{array}{rrrrrrrrrrrrrr}\text { Staff } & 0.6 & 0.6 & 0.6 & 0.6 & 0.5 & 0.6 & 0.6 & 0.6 & 0.6 & 0.6 & 0.5 & 0.6 & 0.6 \\ \text { O4 KE TEP LABO HTS } & 82 & 73 & 75 & 90 & 78 & 82 & 101 & 82 & 77 & 86 & 78 & 96 & 1000\end{array}$

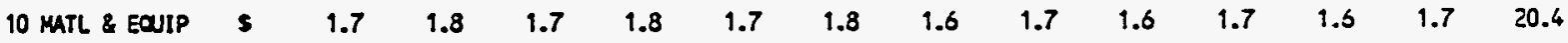

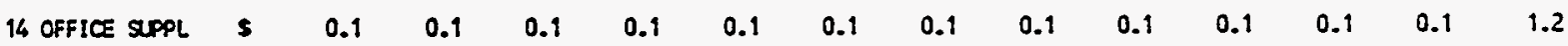

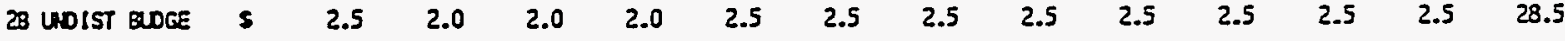

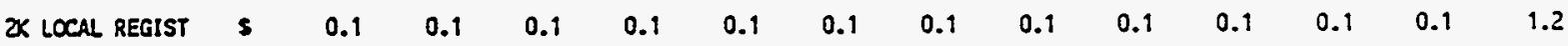

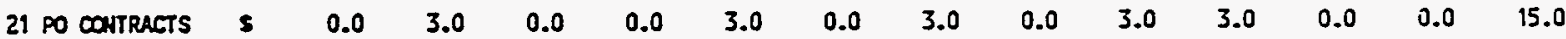




\section{WHC-SP-1116 REV 0}

FOS-8620-R

\begin{tabular}{|c|c|c|c|c|c|c|c|c|c|c|c|c|c|c|}
\hline$\angle C M P R$ & s & 0.4 & 0.6 & 0.3 & 0.3 & 0.7 & 0.4 & 0.7 & 0.4 & 0.6 & 0.6 & 0.6 & 0.6 & 5.8 \\
\hline 4F ОССРАНCY & s & 62.7 & 62.7 & 62.8 & 62.8 & 62.8 & 62.8 & 62.8 & 62.8 & 62.8 & 62.8 & 62.8 & 62.8 & $\pi 33.4$ \\
\hline 4J QUS OPERATIO & s & 0.2 & 0.3 & 0.2 & 0.3 & 0.2 & 0.3 & 0.2 & 0.3 & 0.2 & 0.3 & 0.2 & 0.3 & 3.0 \\
\hline UH LOCKSHITHS & s & 0.5 & 0.4 & 0.5 & 0.4 & 0.5 & 0.4 & 0.5 & 0.4 & 0.4 & 0.6 & 0.4 & 0.4 & 5.2 \\
\hline 4P TRAKSPORTATI & $\mathbf{s}$ & 0.5 & 0.6 & 0.4 & 0.6 & 0.6 & 0.4 & 0.5 & 0.4 & 0.6 & 0.4 & 0.6 & 0.4 & 5.0 \\
\hline 50 DOSIMETRY & $\mathbf{s}$ & 19.5 & 11.6 & 19.6 & 11.6 & 19.6 & 11.6 & 19.6 & 19.6 & 11.6 & 11.6 & 11.6 & 11.6 & 139.1 \\
\hline 5F MANACEMENT $P$ & $\mathbf{s}$ & 8.6 & 8.3 & 8.4 & 8.3 & 8.4 & 8.3 & 8.4 & 8.3 & 8.3 & 8.3 & 8.3 & 8.3 & 100.0 \\
\hline 6A CF COPUTER & 8 & 1.7 & 1.8 & 1.7 & 1.8 & 1.2 & 1.3 & 1.2 & 1.3 & 1.2 & 1.3 & 1.2 & 1.3 & 17.0 \\
\hline 6d ac ASSESSIE & s & 3.8 & 3.7 & 3.8 & 3.7 & 3.8 & 3.7 & 3.8 & 3.7 & 3.7 & 3.7 & 3.7 & 3.7 & 44.8 \\
\hline 4 IRA MAINT SE & s & 1.5 & 1.6 & 1.5 & 1.6 & 1.6 & 1.6 & 1.6 & 9.6 & 1.6 & 1.6 & 1.6 & 1.6 & 19.0 \\
\hline 6N NETLORK ACCE & $\mathbf{s}$ & 5.3 & 5.4 & 5.3 & 5.4 & 5.3 & 5.4 & 5.3 & 5.4 & 5.3 & 5.4 & 5.4 & 5.4 & 64.3 \\
\hline OT TELEPHONE SE & $\mathbf{s}$ & 5.8 & 5.7 & 5.8 & 5.7 & 5.8 & 5.7 & 5.8 & 5.7 & 5.8 & 5.7 & 5.7 & 5.7 & 68.9 \\
\hline$\pi$ CRG OVERKEAD & & 0.2 & 0.2 & 0.2 & 0.2 & 0.2 & 0.2 & 0.3 & 0.2 & 0.2 & 0.2 & 0.2 & 0.3 & 2.6 \\
\hline \multirow[t]{3}{*}{ Total Bas } & Staff & 2.6 & 2.6 & 2.6 & 2.6 & 2.5 & 2.6 & 2.6 & 2.6 & 2.6 & 2.6 & 2.5 & 2.6 & 2.6 \\
\hline & Hrs & 378 & 337 & 347 & 416 & 362 & 380 & 467 & 378 & 355 & 398 & 362 & 444 & 4624 \\
\hline & s & 118.0 & 119.5 & 116.5 & 118.7 & 120.5 & 197.7 & 123.6 & 117.6 & 119.8 & 121.3 & 116.8 & 119.5 & 1429.4 \\
\hline \multirow[t]{2}{*}{$\begin{array}{l}\text { LORK PACXACE: } \\
\text { ReSP Org: }\end{array}$} & \multirow[t]{2}{*}{$\begin{array}{l}\text { IJ801008 } \\
80100 \text { SAF }\end{array}$} & \multirow{2}{*}{\multicolumn{4}{|c|}{$\begin{array}{l}\text { B PROGRAY MANAGEIENT } \\
\text { AFETY ANALYSIS \& ENGINEERING }\end{array}$}} & \multirow{2}{*}{\multicolumn{3}{|c|}{ Scope of Work: }} & & & & & \multicolumn{2}{|c|}{$\begin{array}{l}\text { Revision No: } \infty 0 \\
\text { Date: } 08 / 02 / 94\end{array}$} \\
\hline & & & & & & & & & & & & & \multicolumn{2}{|c|}{ CAP STATUS: $D$} \\
\hline \multicolumn{6}{|c|}{$\begin{array}{l}\text { Task: } 1 \text { 1J8100800 PrOGRAY MALACONOMT } \\
\text { Responsible Org: } \$ 100 \text { SAFETY ANALYSIS \& ENGINEERING }\end{array}$} & $\begin{array}{l}\text { ScOp } \\
\text { PROGR }\end{array}$ & $\begin{array}{l}\text { of Wor } \\
\text { RAA DEVE }\end{array}$ & $\begin{array}{l}\text { k: } \\
\text { LOPMENT }\end{array}$ & IMPLEME & WTATION & & & \multicolumn{2}{|c|}{$\begin{array}{l}\text { Revision No: } 00 \\
\text { Date: 08/02/94 } \\
\text { Cin NUm: } \\
\text { CAP STATUS: } 0\end{array}$} \\
\hline
\end{tabular}

Milestones:

SAE-95-001 R 04/28/95 SARE FY95 DOHSGA7 MID-YEAR REVIEU

SAE-95-002 R 08/31/95 SARE FY 1996 DOH WWGET

SAE-95-003 R 06/30/95 DRAFT OF SAFETY \& ENGINEERING FY '96 SITE

SAE-95-004 X 08/31/95 SARE FY 1996 SITE SUPPCRT PROGRAM PLAH/NULTI

SAE-S5-COS R 04/14/95 AUTOMATED DATA PROCESSING EQUIPMENT MID-YEAR

SAE-S5-006 R 07/28/\% AUTOUTED DATA PROCESSING EQUIPMENT
SUPPCRT PROGRAM PLAH/MULTI-YEAR PROGRAH PLAN

YeAR PROGRAM PLAY

STATEMENT OF STRATEGY FCR FY 1995

PROCREENT PLAN FCR FY 1996 


\section{WHC-SP-1116 REV 0}

FDS-8620-R

SAE-95-007 R 09/15/95 SAFET AMULYSIS \& ENGINEERING MONTHLY PERFCRMAKCE STATISTICS

SAE-95-008 R 09/15/95 SAFETY AKALYSIS \& ENGINEERING SITE MANAGEMETT SYSTEY REPCRT

SAE-95-009 R 09/15/95 SAFETY AMULYSIS \& ENGINEERIMG VARIANCE AND

SAE-95-010 R 09/29/95 SAFETY AMHLYSTS \& ENGIKEERIKG 1JED10 PROCRAM

SAE-95-011 K 01/16/95 QUARTELY PROCRESS REPCRT OF SAFETY AMALYSIS

SAE-95-012 K 04/14/95 QLARTERLY PROGRESS REPCRT OF SAFETY AHALYSIS

SAE-95-013 K. 07/14/95 QUARTERLY PROCRESS REPCRT OF SAFETY ALALYSIS

SAE-95-014 K 09/29/95 CUARTERLY PRDCRESS REPCRT OF SAFETY AHLLYSIS

SAE-95-DRT R 11/15/95 DRAFT OTLIME OF SAFETY ALALYSIS AND

SAE-\$5-022 K 01/15/95 SAFETY AHLLYYSIS AND ENGINEERING TRANSITION PLAN.
CORRECTIVE ACTION REPORT.

DEVELOPMENT AND IMPLEMENTATION PROGRAM REVIEN.

\& ENGIMEERING PROCRAH.

\& ENGINEERING PROGRAM.

AND ENGINEERING

AND ENGINEERING

ENGINEERING TRAMSITION PLAH.
Fiscal Year: 1995

Performing Org: 8100 SAFETY AKLLYSIS \& ENGINEERIMG

Approved By: SCHADE, AR

Cost Elements:

Staff

DO NOH-EXEYPT Hrs

01 EXEPT

Staff
Hrs
$\mathbf{s}$

10 MATL \& EQUIP 5

14 OffICE SUPL

19 COAUTER-HAR

4C MPR

GP MUTINEDIA s s

$\pi$ QRG OVERHEAD

Total BCus staf

$$
\begin{array}{rr}
\text { Staff } & 4.0 \\
\text { Hrs } & 590 \\
5 & 23 .
\end{array}
$$

$9 \quad 198 \quad 204$

2.5

13.6

0.4

0.20 .2

$\begin{array}{llll}.0 & 2.9 & 2.9 & 2.9\end{array}$

$\begin{array}{lll}0.8 & 0.7 & 0.8\end{array}$

$0.8 \quad 0.7$

0.2

$\begin{array}{lll}0.0 & 0.0 \\ 4.0 & 0.0 & 0.0\end{array}$

\begin{tabular}{rrrrr} 
& 590 & 528 & 544 & 653 \\
\hline & 23.8 & 21.6 & 22.1 & 25.6
\end{tabular}

oct Hov Dec Jan

1.5

1.5

$1.5 \quad 1.5$

$1.5 \quad 1$.

$.5 \quad 2.5$

.2

\section{5}

340

14.0

$2.5 \quad 2.5$

$408 \quad 355$

$6-585$

Date: 1,

Feb Mar Apr May

$1.5 \quad 1.5$

$213 \quad 224$

8.4 .0

$5 \quad 2$

373

15.3

2.5
458
18.8

$\begin{array}{rr} & 2.5 \\ 8 & 370 \\ 8 & 15.2\end{array}$

0.40.

$4 \quad 0.5$

$0.5 \quad 0.4$

0.20

0.20 .2

92

.9

3.02

$0.2-0.3$

$0.8 \quad 0.7$

$0.7 \quad 0.8$

$2.9 \quad 2.9$

$2.9 \quad 2.9$

$2.9 \quad 2$

.20 .3

$0.3 \quad 0.2$

0.20 .3

$0.7 \quad 0.7$

$\begin{array}{llll}0.7 & 0.7 & 0.7 & 8.8\end{array}$

.3

$\begin{array}{lllll}0.2 & 0.3 & 0.2 & 0.3 & 3.0\end{array}$

$0.0 \quad 0.0$

$0.0 \quad 0.0$

$0.0 \quad 0.0 \quad 0.0$

$\begin{array}{llll}0.0 & 0.0 & 0.0 & 0.0\end{array}$

Total BCP: 
Task: 1280100801 PROGRAH MaNAGEREMT

Responsible Org: 80100 SAFETY AWAYYSIS \& EMGINEERING

E V Hethod: 4 LEVEL OF EFFCRT HETHCO

Parent LBS:
Scope of Hork:

MEO ACTIVITIES
Revision Ho: 00 Date: 08/02/\%6 Cin Mun: CAP STATUS: D
Fiseal Year: 1995

Perfoming Org: 80100 SAFETY AMALYSIS \& ENGIREERIMG

Approved By: SCHADE, AR

Cost Elements:

26 MISC MEMERS

$\pi$ ORG OVERHEND

Total Bas Staff oct Hov Dec Jan

$0.0 \quad 0.0$

$0.0 \quad 0.0$

$0.0 \quad 0.0$
$0.0 \quad 0.0$

0.0

$0.0 \quad 0.0$
6-5851 Date: $/ /$

Feb Mar Apr May

$0.0 \quad 0.0 \quad 0.0$

$\begin{array}{lll}0.0 & 0.0 & 0.0\end{array}$

$\begin{array}{llll}0.0 & 0.0 & 0.0 & 0.0\end{array}$

$\begin{array}{rrr}0.0 & 0.0 & 0.0 \\ 0 & 0 & 0 \\ 0.0 & 0.0 & 0.0\end{array}$

$\begin{array}{rr}0.0 & 0.0 \\ 0 & 0\end{array}$

$\begin{array}{rr}0 & 0 \\ 0.0 & 0.0\end{array}$
Scope of Hork: EFCOG ACTIVITIES
Revision No: 00 Date: $08 / 02 / 94$ Cin Num: CAP STATUS: D
E V Method: 4 LEVEL OF EFFORT IETHOO

Parene LBS:
Fiscal Year: 1995

Performing Org: D100 SAFETY ANALYSIS \& ENGINEERING

Approved By: SCHADE, AR

\begin{tabular}{|c|c|c|c|c|c|c|c|c|c|c|c|c|c|c|}
\hline \multicolumn{2}{|c|}{ Cost Elements: } & $\infty t$ & Nor & Dec & Jan & Feb & Mar & Apr & May & $\sin$ & Jul. & Aug & Sep & Total \\
\hline & 10 maTL \& EQUIP & 0.0 & 0.0 & 0.1 & 0.0 & 0.0 & 0.2 & 0.0 & 0.1 & 0.0 & 0.0 & 0.1 & 0.0 & 0.5 \\
\hline 2T IRAVEL \& LIV & s & 9.6 & 1.5 & 1.5 & 1.5 & 9.5 & 1.5 & 1.6 & 1.5 & 1.5 & 1.5 & 1.5 & 1.5 & 18.2 \\
\hline 4C MPR & $\mathbf{s}$ & 0.1 & 0.0 & 0.0 & 0.0 & 0.0 & 0.0 & 0.0 & 0.0 & 0.0 & 0.0 & 0.0 & 0.0 & 0.1 \\
\hline 6P MUTINEDIA S & $\mathbf{s}$ & 0.1 & 0.1 & 0.1 & 0.1 & 0.1 & 0.1 & 0.1 & 0.1 & 0.1 & 0.1 & 0.1 & 0.1 & 1.2 \\
\hline \multicolumn{2}{|l|}{$\pi$ CRG OVERHEAD } & 0.0 & 0.0 & 0.0 & 0.0 & 0.0 & 0.0 & 0.0 & 0.0 & 0.0 & 0.0 & 0.0 & 0.0 & 0.0 \\
\hline \multirow[t]{3}{*}{ Total scus } & Staff & 0.0 & 0.0 & 0.0 & 0.0 & 0.0 & 0.0 & 0.0 & 0.0 & 0.0 & 0.0 & 0.0 & 0.0 & 0.0 \\
\hline & Hrs & 0 & 0 & 0 & 0 & 0 & 0 & 0 & 0 & 0 & 0 & 0 & 0 & 0 \\
\hline & $\mathbf{s}$ & 1.8 & 1.6 & 1.7 & 1.6 & 1.6 & 1.8 & 1.7 & 1.7 & 1.6 & 9.6 & 1.7 & 1.6 & 20.0 \\
\hline
\end{tabular}

Total BCP:

Task: 1J80100803 PROGRAM MAYACER

Responsible Org: 8100 SAFETY AMLIYSIS \& EMGINEERIHG

$E V$ Hethod: 4 LEVEL OF EFFCRT VETHCO

Parent LaS:
Scope of Hork:

PROF. SOC. \& STOS GROPS
Revision No: 00 Date: 08/02/9/4 Cin Num: CAP STATUS: 0

Fiscal Year: 1995 
WHC-SP-1116 REV 0

FOS-BS20-R

Performin Org: 80100 SAFETY AULCYYSIS \& EMGINEERIHG

Approved By: SCHADE, AR

6-5851 Date: $/ 1$

\begin{tabular}{|c|c|c|c|c|c|c|c|c|c|c|c|c|c|c|}
\hline \multicolumn{2}{|l|}{ Cost Elements: } & oct & Hov & Dec & $\operatorname{Jan}$ & Feb & $\mathrm{Mar}$ & Apr & May & Jun & Jul & Aug & Sep & Total \\
\hline 2T TRAVEL \& LIV & s & 0.0 & 2.0 & 0.0 & 2.0 & 0.0 & 0.0 & 0.0 & 2.0 & 0.0 & 1.0 & 0.0 & 2.0 & 9.0 \\
\hline $2 X$ PROF\&TECH SO & $\$$ & 0.0 & 2.0 & 0.0 & 2.0 & 0.0 & 2.0 & 0.0 & 1.5 & 0.0 & 2.0 & 0.0 & 1.5 & \\
\hline \multicolumn{2}{|l|}{$\pi$ CRG OVERHEAD } & 0.0 & 0.0 & 0.0 & 0.0 & 0.0 & 0.0 & 0.0 & 0.0 & 0.0 & 0.0 & 0.0 & 0.0 & 0.0 \\
\hline \multirow[t]{3}{*}{ Total Bous } & Staff & 0.0 & 0.0 & 0.0 & 0.0 & 0.0 & 0.0 & 0.0 & 0.0 & 0.0 & 0.0 & 0.0 & 0.0 & .0 \\
\hline & Hrs & 0 & 0 & 0 & 0 & 0 & 0 & 0 & 0 & 0 & 0 & 0 & 0 & 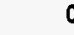 \\
\hline & s & 0.0 & 4.0 & 0.0 & 4.0 & 0.0 & 2.0 & 0.0 & 3.5 & 0.0 & 3.0 & 0.0 & 3.5 & 20.0 \\
\hline
\end{tabular}

Total BCP:

\section{Task: 1J80100804 PROGRAM HANAEER}

Responsible Org: 80100 SAFETY AMALYSIS \& EMGIHEERING

E V Hethod: 4 LEVEL OF EFFCRT VETHOD

Parent LBS:

\section{Scope of Work:}

CONTRACTS ADMINISTRATIOH
Revision No: 00 Date: $08 / 02 / 94$

Cin Num:

CAP STATUS: 0

Milestones: Marrative

SAE-95-016 R 09/26/95 YOWTHLY STATUS REPCRT OF SLBCOHTRACTCR SUPPORT.

SAE-95-017 X 01/16/95 QURTERLY OITSOURCING STATUS REPCRT.

SAE-\$S-018 K 04/14/\%5 OUARTERLY OUTSOURCING STATUS REPCRT.

SAE-95-019 K 07/14/9S QUARTERLY CUTSOURCIHG STATUS REPCRT.

SAE-95-020 K 09/29/95 QUARTERLY OITSOURCING STATUS REPCRT.

\begin{tabular}{|c|c|c|c|c|c|c|c|c|c|c|c|c|c|c|}
\hline \multicolumn{15}{|c|}{ Fiscal Year: 1995} \\
\hline \multirow{2}{*}{\multicolumn{2}{|c|}{$\begin{array}{l}\text { Performing Org: } 80100 \\
\text { Approved By: } \\
\text { Cost Elements: }\end{array}$}} & \multirow[b]{2}{*}{ oct } & \multicolumn{3}{|c|}{ — SCHADE, AR } & \multicolumn{2}{|c|}{$6-5851$} & \multirow{2}{*}{$\begin{array}{l}\text { Date: } \\
\text { Apr }\end{array}$} & \multirow{2}{*}{$\begin{array}{l}1 \\
\text { May }\end{array}$} & \multirow[b]{2}{*}{ Jun } & \multirow[b]{2}{*}{ Jul } & \multirow[b]{2}{*}{ Aug } & \multirow[b]{2}{*}{ Sep } & \multirow[b]{2}{*}{ Total } \\
\hline & & & Hor & Dec & Jan & Feb & Mar & & & & & & & \\
\hline \multirow{3}{*}{01 EXEPT } & Staff & 2.0 & 2.0 & 2.0 & 2.0 & 2.0 & 2.0 & 2.0 & 2.0 & 2.0 & 2.0 & 2.0 & 2.0 & 2.0 \\
\hline & Hirs & 296 & 264 & 272 & 326 & 284 & 298 & 366 & 296 & 278 & 312 & 284 & 348 & 3624 \\
\hline & s & 12.2 & 10.8 & 11.2 & 13.4 & 11.7 & 12.2 & 15.0 & 12.2 & 11.4 & 12.8 & 11.7 & 14.3 & 148.9 \\
\hline \multirow{3}{*}{ OS HE PARTTIKE } & Staff & 1.0 & 1.0 & 1.0 & 1.0 & 1.0 & 1.0 & 1.0 & 1.0 & 1.0 & 1.0 & 1.0 & 1.0 & 1.0 \\
\hline & Hrs & 148 & 132 & 136 & 163 & 142 & 149 & 183 & 148 & 139 & 156 & 142 & 174 & 1812 \\
\hline & s & 2.6 & 2.3 & 2.4 & 2.9 & 2.5 & 2.6 & 3.2 & 2.6 & 2.5 & 2.8 & 2.5 & 3.1 & 32.1 \\
\hline \multicolumn{2}{|l|}{$\pi$ ORG OVERHEND } & 0.0 & 0.0 & 0.0 & 0.0 & 0.0 & 0.0 & 0.0 & 0.0 & 0.0 & 0.0 & 0.0 & 0.0 & 0.0 \\
\hline \multirow[t]{3}{*}{ Total BCus } & Staff & 3.0 & 3.0 & 3.0 & 3.0 & 3.0 & 3.0 & 3.0 & 3.0 & 3.0 & 3.0 & 3.0 & 3.0 & 3.0 \\
\hline & Hrs & 444 & 396 & 408 & 489 & 426 & 447 & 549 & 446 & 417 & 468 & 426 & 522 & 5436 \\
\hline & s & 14.8 & 13.2 & 13.6 & 16.3 & 14.2 & 14.9 & 18.3 & 14.8 & 13.9 & 15.6 & 14.2 & 17.4 & 181.0 \\
\hline
\end{tabular}

Total BCP: 


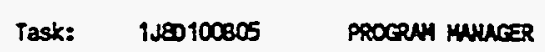

Responsible Org: 80100 SAFETY AWLLYSIS \& ENGINEERIMG

E V Hethod: 4 LEVEL OF EFFORT HETHOD

Parent bis:
Scope of Hork:

CAM AOMINISTRATIOAS
Revision Ho: $\infty$ Dare: $08 / 02 / 94$

Cin Num: CAP STATUS: 0

Fiseal Year: 1995

Performing org: 80100 SAFETY ANLLYSIS \& ENGINEERING

Approved By: SCHADE, AR

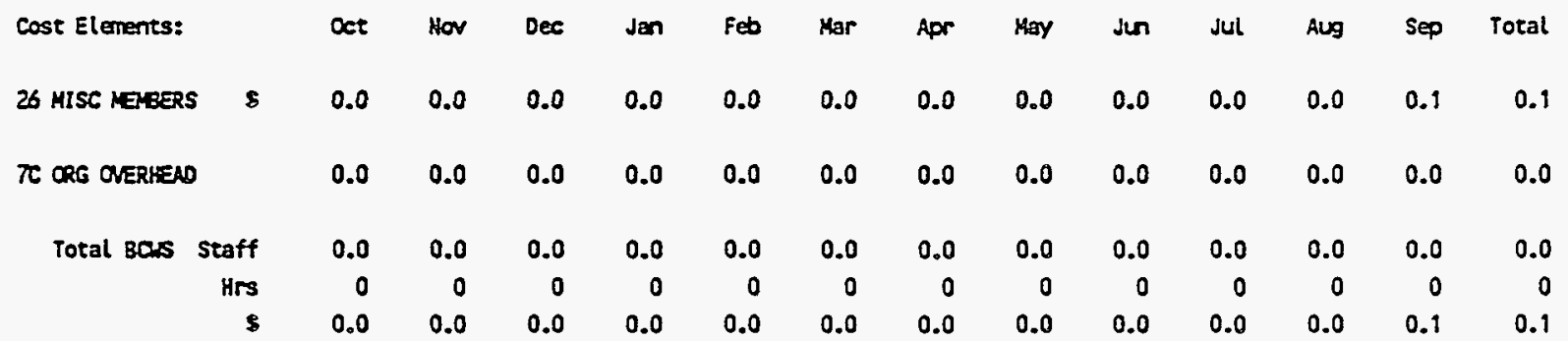

Total 8ap:

Task: 1J80100806 PROGRAH MAMAGER

RESPonsible Org: 80100 SAFETY ANALYSIS \& EMGIHEERIMG

E V Hethod: \& LEVEL OF EFFORT vETHOD

Parent ths:

Mi lestones:

Narracive

SAE- $55-007$ R 12/30/94 SA8E SAFETY ANALYSIS PROGRAM PLAN UPOATE

SAE-95-008 \& 06/30/55 SAFETY AMALYSIS \& ENGINEERING SAFETY AMALYSIS PROGRAM PLAN UPOATE
Scope of Work:

SAFETY ANQLYSIS PROGRAM PLAY
Revision Ho: 00 Date: $08 / 02 / \%$ Cin Hum: CAP STATUS: 0

Performing arg: 80100 SAFETY ANALYSIS \& ENGINEERING

Approved By: SCHADE, AR

6-5851 Date: / /

\begin{tabular}{|c|c|c|c|c|c|c|c|c|c|c|c|c|c|c|}
\hline Cost Elements: & & $\infty c t$ & Hor & Dec & Jan & Feb & Mar & Apr & May & Jun & Jul & $A \cup g$ & Sep & Total \\
\hline 26 MISC MEMERS & $s$ & 0.0 & 0.0 & 0.0 & 0.0 & 0.0 & 0.0 & 0.0 & 0.0 & 0.0 & 0.0 & 0.0 & 0.1 & 0.1 \\
\hline C ORG OVERHEAD & & 0.0 & 0.0 & 0.0 & 0.0 & 0.0 & 0.0 & 0.0 & 0.0 & 0.0 & 0.0 & 0.0 & 0.0 & 0.0 \\
\hline Total BCus & Staff & 0.0 & 0.0 & 0.0 & 0.0 & 0.0 & 0.0 & 0.0 & 0.0 & 0.0 & 0.0 & 0.0 & 0.0 & 0.0 \\
\hline & Hrs & 0 & 0 & 0 & 0 & 0 & 0 & 0 & 0 & 0 & 0 & 0 & 0 & 0 \\
\hline & $\mathbf{s}$ & 0.0 & 0.0 & 0.0 & 0.0 & 0.0 & 0.0 & 0.0 & 0.0 & 0.0 & 0.0 & 0.0 & 0.1 & 0.1 \\
\hline
\end{tabular}

Total BCP:

\begin{tabular}{|c|c|c|c|c|c|c|c|c|c|c|c|c|c|c|}
\hline \multirow[t]{2}{*}{ Total } & & $\infty<t$ & Hor & Dec & Jan & $F \oplus b$ & Mar & Apr & May & Jun & Jul & Aus & Sep & Total \\
\hline & Staff & 1.5 & 1.5 & 1.5 & 1.5 & 1.5 & 1.5 & 1.5 & 1.5 & 1.5 & 1.5 & 1.5 & 1.5 & 1.5 \\
\hline \multirow[t]{3}{*}{ D MON-EXEMPT } & Hrs & 220 & 198 & 204 & 245 & 213 & 224 & 275 & 222 & 200 & 234 & 213 & 261 & 2718 \\
\hline & 5 & 3.9 & 3.5 & 3.6 & 4.3 & 3.8 & 4.0 & 4.9 & 3.9 & 3.7 & 4.1 & 3.8 & 4.6 & 48.2 \\
\hline & Staff & 4.5 & 4.5 & 4.5 & 4.5 & 4.5 & 4.5 & 4.5 & 4.5 & 4.5 & 4.5 & 4.5 & 4.5 & 4.5 \\
\hline
\end{tabular}


FOS-8620-R

WHC-SP-1116 REV 0

Financial Data Systen

$09 / 14 / 94,14: 22$

COST ACOONT PLAN - TJ8010 - VERSION 1995

Page: 9

\begin{tabular}{|c|c|c|c|c|c|c|c|c|c|c|c|c|c|c|}
\hline \multirow[t]{2}{*}{01 EXaPT } & $\begin{array}{r}\text { Hirs } \\
\text { s }\end{array}$ & $\begin{array}{r}666 \\
27.4\end{array}$ & $\begin{array}{r}594 \\
24.4\end{array}$ & $\begin{array}{r}612 \\
25.1\end{array}$ & $\begin{array}{r}734 \\
30.1\end{array}$ & $\begin{array}{r}639 \\
26.2\end{array}$ & $\begin{array}{r}671 \\
27.6\end{array}$ & $\begin{array}{r}824 \\
33.8\end{array}$ & $\begin{array}{r}666 \\
27.4\end{array}$ & $\begin{array}{r}626 \\
25.7\end{array}$ & $\begin{array}{r}702 \\
28.8\end{array}$ & $\begin{array}{r}639 \\
26.2\end{array}$ & $\begin{array}{r}783 \\
32.2\end{array}$ & $\begin{array}{r}8156 \\
335.0\end{array}$ \\
\hline & Staff & 1.0 & 1.0 & 1.0 & 1.0 & 1.0 & 1.0 & 1.0 & 1.0 & 1.0 & 1.0 & 1.0 & 1.0 & 1.0 \\
\hline \multirow[t]{2}{*}{ OS ME PARTTIIE } & Hirs & 148 & 132 & 136 & 163 & 142 & 149 & 183 & 148 & 139 & 156 & 142 & 174 & 1812 \\
\hline & $\mathbf{s}$ & 2.6 & 2.3 & 2.4 & 2.9 & 2.5 & 2.6 & 3.2 & 2.6 & 2.5 & 2.8 & 2.5 & 3.1 & 32.1 \\
\hline 10 MATL \& EOUIP & $\$$ & 0.5 & 0.4 & 0.5 & 0.4 & 0.4 & 0.6 & 0.5 & 0.5 & 0.4 & 0.4 & 0.5 & 0.4 & 5.5 \\
\hline 14 OffICE SUPPL & $s$ & 0.2 & 0.2 & 0.2 & 0.2 & 0.2 & 0.2 & 0.2 & 0.2 & 0.2 & 0.2 & 0.2 & 0.2 & 2.6 \\
\hline 19 COMUTER-HAR & $\mathbf{s}$ & 3.0 & 2.9 & 2.9 & 2.9 & 2.9 & 2.9 & 3.0 & 2.9 & 2.9 & 2.9 & 2.9 & 2.9 & 35.0 \\
\hline 2T TRAVEL \& LIV & $s$ & 1.6 & 3.5 & 9.5 & 3.5 & 1.5 & 1.5 & 1.6 & 3.5 & 1.5 & 2.5 & 1.5 & 3.5 & 27.2 \\
\hline $2 X$ PROF\&TECH $5 O$ & s & 0.0 & 2.0 & 0.0 & 2.0 & 0.0 & 2.0 & 0.0 & 1.5 & 0.0 & 2.0 & 0.0 & 1.5 & 11.0 \\
\hline 26 HISC MEYBERS & $\mathbf{s}$ & 0.0 & 0.0 & 0.0 & 0.0 & 0.0 & 0.0 & 0.0 & 0.0 & 0.0 & 0.0 & 0.0 & 0.3 & 0.3 \\
\hline LC MPR & $\mathbf{s}$ & 0.9 & 0.7 & 0.8 & 0.7 & 0.8 & 0.7 & 0.8 & 0.7 & 0.7 & 0.7 & 0.7 & 0.7 & 8.9 \\
\hline GP MLTIMEDIA S & $\mathbf{s}$ & 0.3 & 0.4 & 0.3 & 0.4 & 0.3 & 0.4 & 0.3 & 0.4 & 0.3 & 0.4 & 0.3 & 0.4 & 4.2 \\
\hline$\pi$ CRG OVERREEAD & & 0.0 & 0.0 & 0.0 & 0.0 & 0.0 & 0.0 & 0.0 & 0.0 & 0.0 & 0.0 & 0.0 & 0.0 & 0.0 \\
\hline \multirow[t]{3}{*}{ Total BCus } & Staff & 7.0 & 7.0 & 7.0 & 7.0 & 7.0 & 7.0 & 7.0 & 7.0 & 7.0 & 7.0 & 7.0 & 7.0 & 7.0 \\
\hline & Hrs & 1034 & 924 & 952 & 1142 & 994 & 1044 & 1282 & 1036 & 974 & 1092 & 994 & 1218 & 12686 \\
\hline & $\boldsymbol{s}$ & 40.4 & 40.3 & 37.4 & 47.5 & 38.6 & 42.5 & 48.4 & 43.6 & 37.9 & 44.8 & 38.6 & 49.8 & 509.8 \\
\hline
\end{tabular}

$\begin{array}{llll}\text { WORK PACXACE: } & \text { 1J80100C SITE SUPPCRT ACTIVITIES } & \text { SOODe of Work: } & \text { Revision No: } 00 \\ \text { Resp Org: } & 80100 \text { SAFETY ARALYSIS \& ENGINEERING } & \text { SITE SLPPCRT ACTIVITES } & \text { Date: 08/02/94 }\end{array}$

Resp Org: $\quad 80100$ SAFETY AKALYSIS \& ENGIMEERIMG SITE SUPPCRT ACTIVITES

CAP STATUS: 0

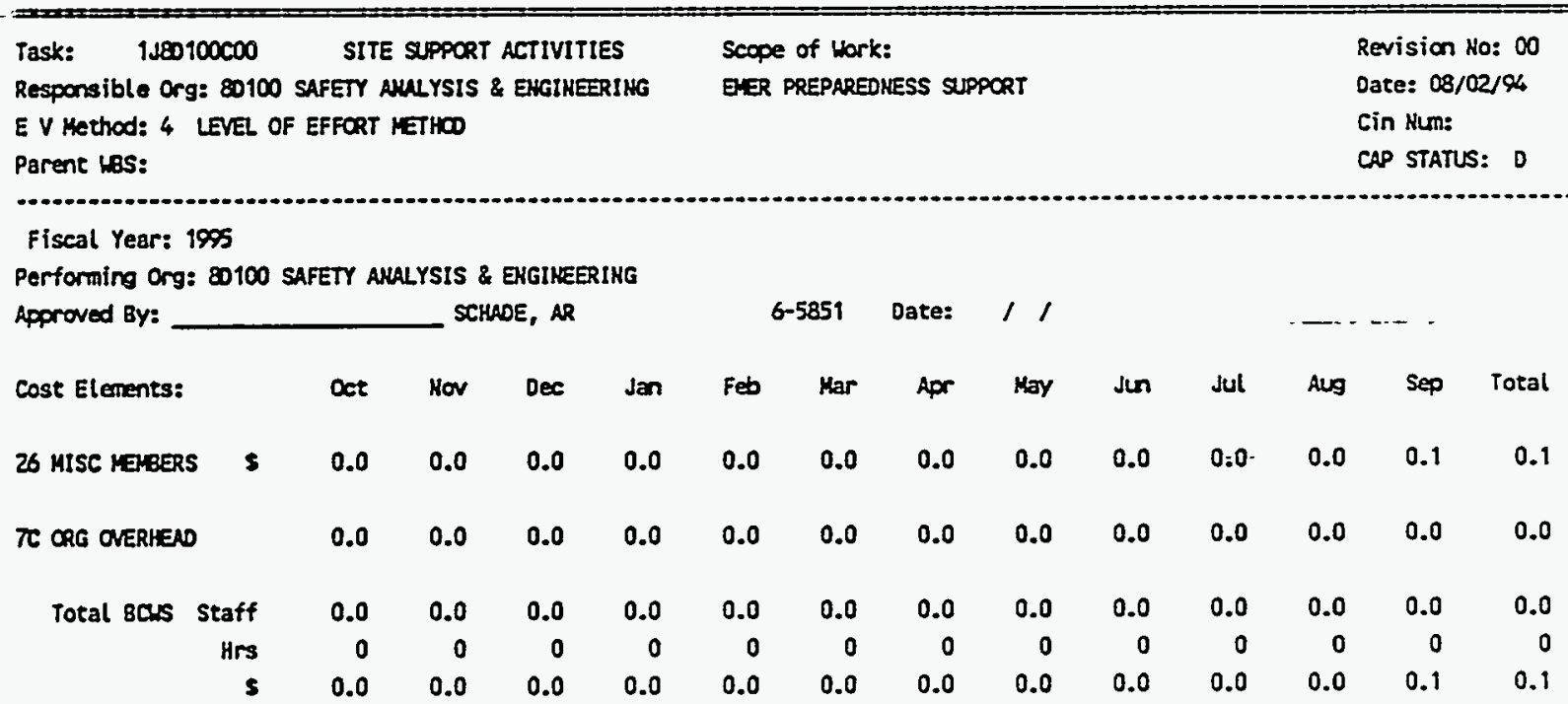

Total BCD: 


\section{WHC-SP-1116 REV 0}

FDS-B620-R

Task: 1 1J80100COI SITE SUPPCRT ACTIVITIES Responsible Org: 8D100 SAFETY AHLLYSIS \& ENGINEERING E V Hethod: 4 LEVEL OF EFFORT VETHOO Parent LBS:
Scope of Hork:

SEAC ACTIVITIES \& SUPPCRT
Revision No: $\infty$ Date: $08 / 02 / 9 / 4$

Cin Nem:

CAP STATUS: 0

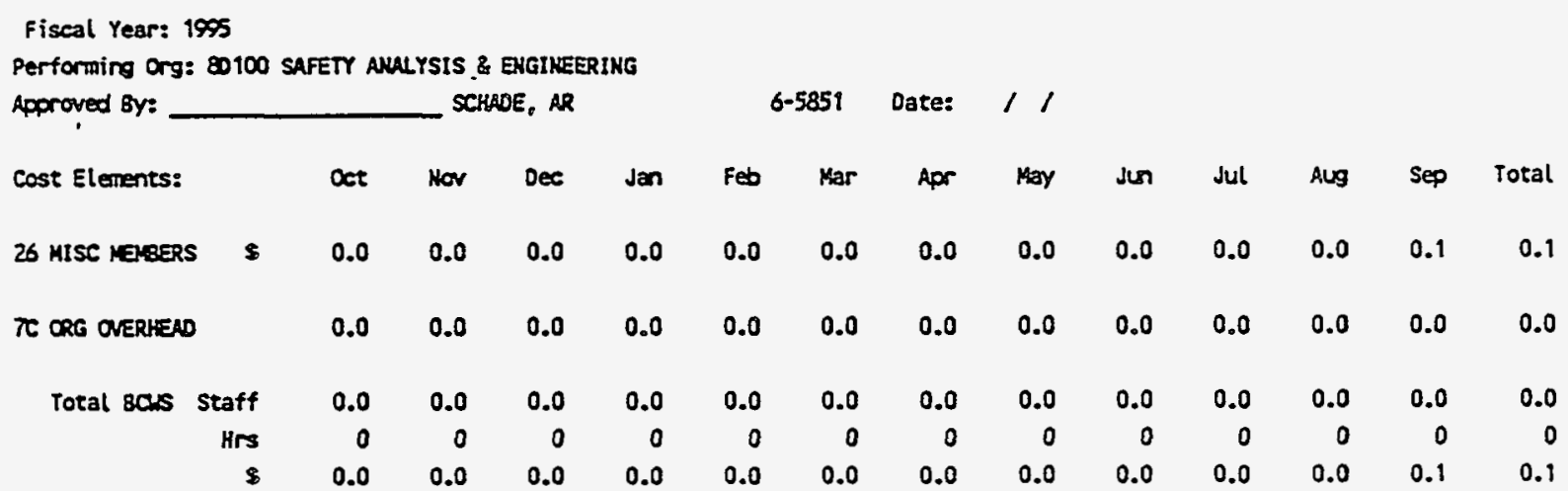

Total BCP:

Total ip Qet Nor Dee Jen Feb Mar Apr May jun Jul sing sep Total

26 YISC MEMERS

$\boldsymbol{s}$

$0.0 \quad 0.0$

0.0

0.0

$\pi$ CRG OVERHEAD

$0.0 \quad 0.0 \quad 0$.

0.0

$0.0 \quad 0.0$

$0.0 \quad 0.0$

0.0

$0.0 \quad 0.0$

$0.0 \quad 0.0$

Total BCus Staff

$\begin{array}{rr}0.0 & 0.0 \\ 0 & 0 \\ 0.0 & 0.0\end{array}$

$\begin{array}{rr}0.0 & 0.0 \\ 0 & 0 \\ 0.0 & 0.0\end{array}$

$\begin{array}{rr}0.0 & 0.0 \\ 0 & 0 \\ 0.0 & 0.0\end{array}$

$0.0 \quad 0.0$

$\begin{array}{lllll}0.0 & 0.0 & 0.0 & 0.0 & 0.0\end{array}$

Hrs

$\begin{array}{rrrrrrr}0.0 & 0.0 & 0.0 & 0.0 & 0.0 & 0.0 & 0.0 \\ 0 & 0 & 0 & 0 & 0 & 0 & 0\end{array}$

$\begin{array}{rr}0 & 0 \\ 0.0 & 0.0\end{array}$

$$
0.0
$$

0.0

$\begin{array}{lll}0.0 & 0.2 & 0.2\end{array}$

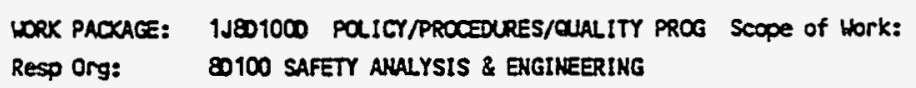

Revision Ho: 00 Date: $08 / 02 / 94$

CAP STATUS: D

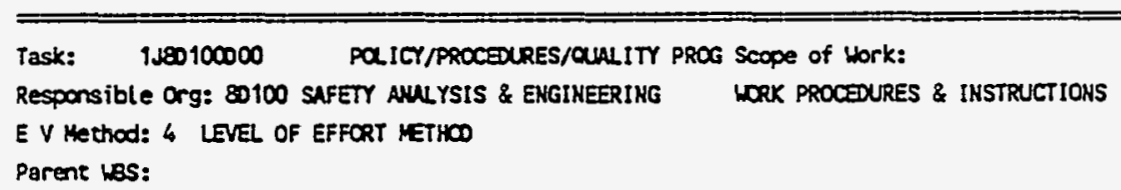

Milestones: Marrative

SAE-S5-018 R 09/29/95 SAFETY ANQUYSIS \& ENGINEERING LDRK PRDCEDURES LPOATE

Fiscal Year: 1995

Perforning Org: 80100 SAFETY AHALYSIS \& ENGIKEERIMG

Approved By: SCHADE, AR

Cost Elements:

Det Nov Dec Jan

26 MISC NERERS

$0.0 \quad 0$.

$0.0 \quad 0.0$

$0.0 \quad 0$.

$0.0 \quad 0.0$

Overheads, Safety Analysis \& Engineering Page 3-40
Revision No: 00 Date: 08/02/94

Cin Num: CAP STATUS: 0
TC ORG OVERHEAD

$0.0 \quad 0.0$




\section{WHC-SP-1116 REV 0}

FDS-8620-R

Financial Data Systen

$09 / 14 / 94$ 14:22

COST ACOONNT PLAH - 1J8010 - VERSIOH 1995

Page: 11

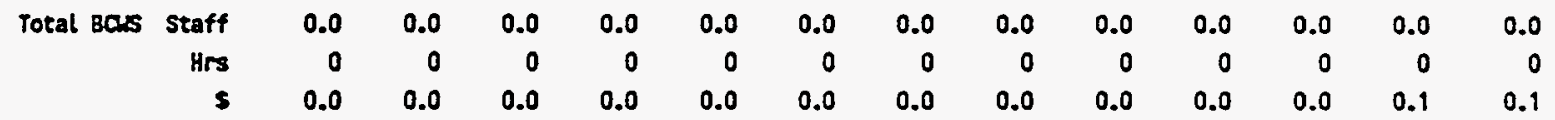

Total BCP:

Task: IJ80100001 POLICY/PROCEDURES/OULLITY PROG SCOPE of Work:

Responsible Org: 8D100 SAFETY AHULYSIS \& EMGIHEERING QULITY ASSR PRDGRAM PLAH

E V Method: 4 LEVEL OF EFFCRT FETHCO

Parent los:
Revision No: 00 Date: $08 / 02 / 96$ Cin Num:

CAP STATUS: D

Milestones:

Marrative

SAE-95-019 R 06/30/\%5 SAFETY ANULYSIS \& ENGINEERING QLALITY ASSURAHCE PROGRAH PLAN UPDATE

Fiseal Year: $19 \% 5$

Performin Org: 80100 SAFETY AMALYSIS \& ENGINEERING

Approved By: SCHAOE, AR

Cost Elements:

26 HISC MERERS

$\pi$ ORG OVERHEN

Total Bas staff

$\begin{array}{rrr}\text { Staff } & 0.0 \\ \text { S } & 0.0\end{array}$

cet Hor Deces Jan

$\begin{array}{lll}0.0 & 0.0 & 0.0\end{array}$

0.0

$0.0 \quad 0.0$

$0.0 \quad 0.0$

0.0
0
0.0

$\begin{array}{rr}0.0 & 0.0 \\ 0 & 0 \\ 0.0 & 0.0\end{array}$

$\begin{array}{rr}0.0 & 0.0 \\ 0 & 0 \\ 0.0 & 0.0\end{array}$

$6-5851$

Date:

$1 /$

Total BAP:

\section{Task: IID100002 POLICY/PROCEDURES/QULLITT PROS SCOpe of Work:}

Responsible Org: 80100 SAFETY AHALYSIS \& ENGIMEERIHG SARE SAFETY PROCRAH

E V Method: 4 LEVEL OF EFFORT METHOD

Parent HBS:

Fiseal Year: $19 \% 5$

Performing Org: 80100 SAFETY ARULYSIS \& ENGIKEERIMG

Approved By: SCHAOE, AR 6.5851 Date: $/ /$

Cost Elements: Det Hov Dec Jan feb Mar Apr Ma

\begin{tabular}{|c|c|c|c|c|c|c|c|c|c|c|c|c|c|}
\hline 26 MISC HaBBERS & s & 0.0 & 0.0 & 0.0 & 0.0 & 0.0 & 0.0 & 0.0 & 0.0 & 0.0 & 0.0 & 0.0 & 0.1 \\
\hline$\pi$ ORG OVERHEAD & & 0.0 & 0.0 & 0.0 & 0.0 & 0.0 & 0.0 & 0.0 & 0.0 & 0.0 & 0.0 & 0.0 & 0.0 \\
\hline Total BCus & Staff & 0.0 & 0.0 & 0.0 & 0.0 & 0.0 & 0.0 & 0.0 & 0.0 & 0.0 & 0.0 & 0.0 & 0.0 \\
\hline & Hrs & 0 & 0 & 0 & 0 & 0 & 0 & 0 & 0 & 0 & 0 & 0 & 0 \\
\hline & s & 0.0 & 0.0 & 0.0 & 0.0 & 0.0 & 0.0 & 0.0 & 0.0 & 0.0 & 0.0 & 0.0 & 0.1 \\
\hline
\end{tabular}

Total BCD: 


\begin{tabular}{|c|c|c|c|c|c|c|c|c|c|c|c|c|c|c|}
\hline Total ip & & $\infty<t$ & Nov & DeC & Jan & Feb & Her & Apr & Kay & Jun & Jut & Aug & sep & Total \\
\hline 26 MISC MEBERS & s & 0.0 & 0.0 & 0.0 & 0.0 & 0.0 & 0.0 & 0.0 & 0.0 & 0.0 & 0.0 & 0.0 & 0.3 & 0.3 \\
\hline$\pi$ ORG OVERHEAD & & 0.0 & 0.0 & 0.0 & 0.0 & 0.0 & 0.0 & 0.0 & 0.0 & 0.0 & 0.0 & 0.0 & 0.0 & 0.0 \\
\hline Total BCSS & Staff & 0.0 & 0.0 & 0.0 & 0.0 & 0.0 & 0.0 & 0.0 & 0.0 & 0.0 & 0.0 & 0.0 & 0.0 & 0.0 \\
\hline & Hrs & 0 & 0 & 0 & 0 & 0 & 0 & 0 & 0 & 0 & 0 & 0 & 0 & 0 \\
\hline & 5 & 0.0 & 0.0 & 0.0 & 0.0 & 0.0 & 0.0 & 0.0 & 0.0 & 0.0 & 0.0 & 0.0 & 0.3 & 0.3 \\
\hline \multirow[t]{2}{*}{$\begin{array}{l}\text { LORK PACXACE: } \\
\text { Resp Org: }\end{array}$} & \multirow{2}{*}{\multicolumn{5}{|c|}{$\begin{array}{l}\text { IJ80100E OLALIFICATIONS \& TRUIHING } \\
\text { \& } 100 \text { SAFETY ANULYSIS \& ENGIKEERING }\end{array}$}} & \multirow{2}{*}{\multicolumn{3}{|c|}{ Scope of Work: }} & & & $=$ & \multicolumn{3}{|c|}{$\begin{array}{l}\text { Revision No: } \infty \\
\text { Date: } 08 / 02 / \% 4\end{array}$} \\
\hline & & & & & & & & & & & & & AP STATL & $: 0$ \\
\hline \multirow{2}{*}{$\begin{array}{l}\text { Task: } 9180 \\
\text { Responsible or } \\
\text { E V Method: } 4 \\
\text { Parent LBS: }\end{array}$} & $\begin{array}{l}\text { 100ED } \\
\text { 9: } 80100 \text { SA } \\
\text { LEVEL OF E }\end{array}$ & \multicolumn{4}{|c|}{$\begin{array}{l}\text { QUALIFICATIOUS \& TRAIHIMG } \\
\text { IFETY AWLYSIS \& ENGIMEERIHG }\end{array}$} & \multirow{2}{*}{\multicolumn{3}{|c|}{$\begin{array}{l}\text { Scope of Work: } \\
\text { MADATCRY TRAINIHG }\end{array}$}} & & & & \multirow{2}{*}{\multicolumn{3}{|c|}{$\begin{array}{l}\text { Revision No: } 00 \\
\text { Dare: } 08 / 02 / 94 \\
\text { Cin Num: } \\
\text { CAP STATUS: D }\end{array}$}} \\
\hline & & & & & & & & & & & & & & \\
\hline
\end{tabular}

Milestones: Marrative

SAE- 5 -015 R 08/31/95 SAFETY AMALYSIS \& ENGINEERING AMRUAL UPDATE OF TRAINING

Fiscal Year: 1995

Performing Org: 80100 SAFETY AVULYSIS \& EMGINEERING

Approved By: SCHADE, AR

6-5851 Date: $/ /$

\begin{tabular}{|c|c|c|c|c|c|c|c|c|c|c|c|c|c|c|}
\hline \multicolumn{2}{|l|}{ Cost Elements: } & $\infty c t$ & Hov & Dec & Jan & Feb & Mar & Apr & May & Jun & Jul & Aug & Sep & Total \\
\hline 2L TRAIHING-ONS & s & 0.7 & 0.8 & 0.8 & 0.8 & 0.8 & 0.8 & 0.8 & 0.8 & 0.8 & 0.8 & 0.8 & 0.8 & 9.5 \\
\hline 41 TRHG-SAFEZEN & s & 0.2 & 0.2 & 0.2 & 0.2 & 0.2 & 0.2 & 0.2 & 0.2 & 0.2 & 0.2 & 0.2 & 0.2 & 2.4 \\
\hline 46 OTRC TRAIHIK & $\mathbf{s}$ & 0.2 & 0.2 & 0.2 & 0.2 & 0.2 & 0.2 & 0.2 & 0.2 & 0.2 & 0.2 & 0.2 & 0.3 & 2.5 \\
\hline$\pi$ CRG OVERHEAD & & 0.0 & 0.0 & 0.0 & 0.0 & 0.0 & 0.0 & 0.0 & 0.0 & 0.0 & 0.0 & 0.0 & 0.0 & 0.0 \\
\hline Total Bcus & Staff & 0.0 & 0.0 & 0.0 & 0.0 & 0.0 & 0.0 & 0.0 & 0.0 & 0.0 & 0.0 & 0.0 & 0.0 & 0.0 \\
\hline & Hrs & 0 & 0 & 0 & 0 & 0 & 0 & 0 & 0 & 0 & 0 & 0 & 0 & 0 \\
\hline & 5 & 1.1 & 1.2 & 1.2 & 1.2 & 1.2 & 1.2 & 1.2 & 1.2 & 1.2 & 1.2 & 1.2 & 1.3 & 4.4 \\
\hline
\end{tabular}

Total Bap:

\section{Task: 1J80100E01 QLLLIFICATION \& TRAINING} Responsible Org: 80100 SAFETY ANALYSIS \& EMGIHEERIHG E V Method: 4 LEVEL OF EFFORT METHOD Parent LBS: TECHNICAL TRAINING
Revision Ho: 00 Date: $08 / 02 / 94$

Cin NUM: CAP STATUS:

Fiscal Year: 1995 
Performing org: BD100 SAFETY AHALYSIS \& EKGIMEERIHG

Approved By: SCHADE, AR

Cost Elements:

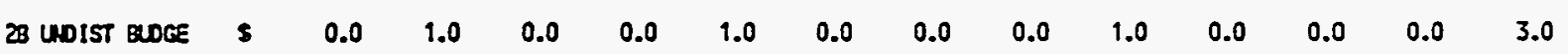

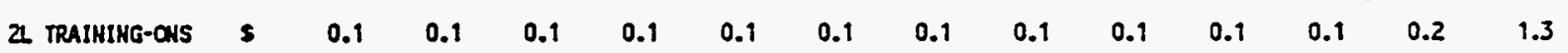

TC ORG OVERHEAD

Total BCSS Staf $\begin{array}{llll}0.0 & 0.0 & 0.0 & 0.0\end{array}$

$0.0 \quad 0.0$

$0.0 \quad 0.0$

$\begin{array}{rrrrrrr}0.0 & 0.0 & 0.0 & 0.0 & 0.0 & 0.0 & 0.0 \\ 0.0 & 0.0 & 0.0 & 0.0 & 0.0 & 0.0 & 0.0 \\ 0 & 0 & 0 & 0 & 0 & 0 & 0 \\ 0.1 & 0.1 & 1.1 & 0.1 & 0.1 & 0.2 & 4.3\end{array}$

Total Bap:

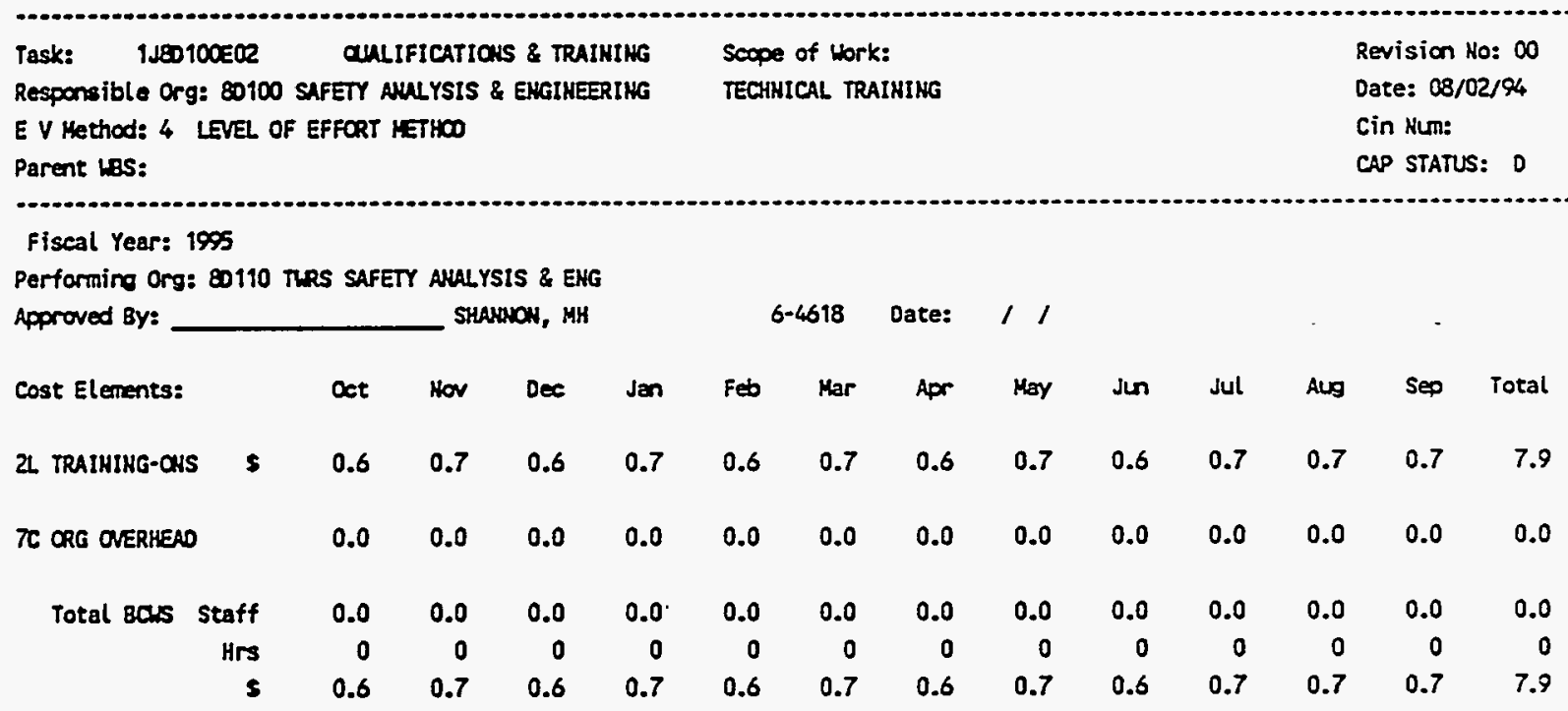

Total BCP:

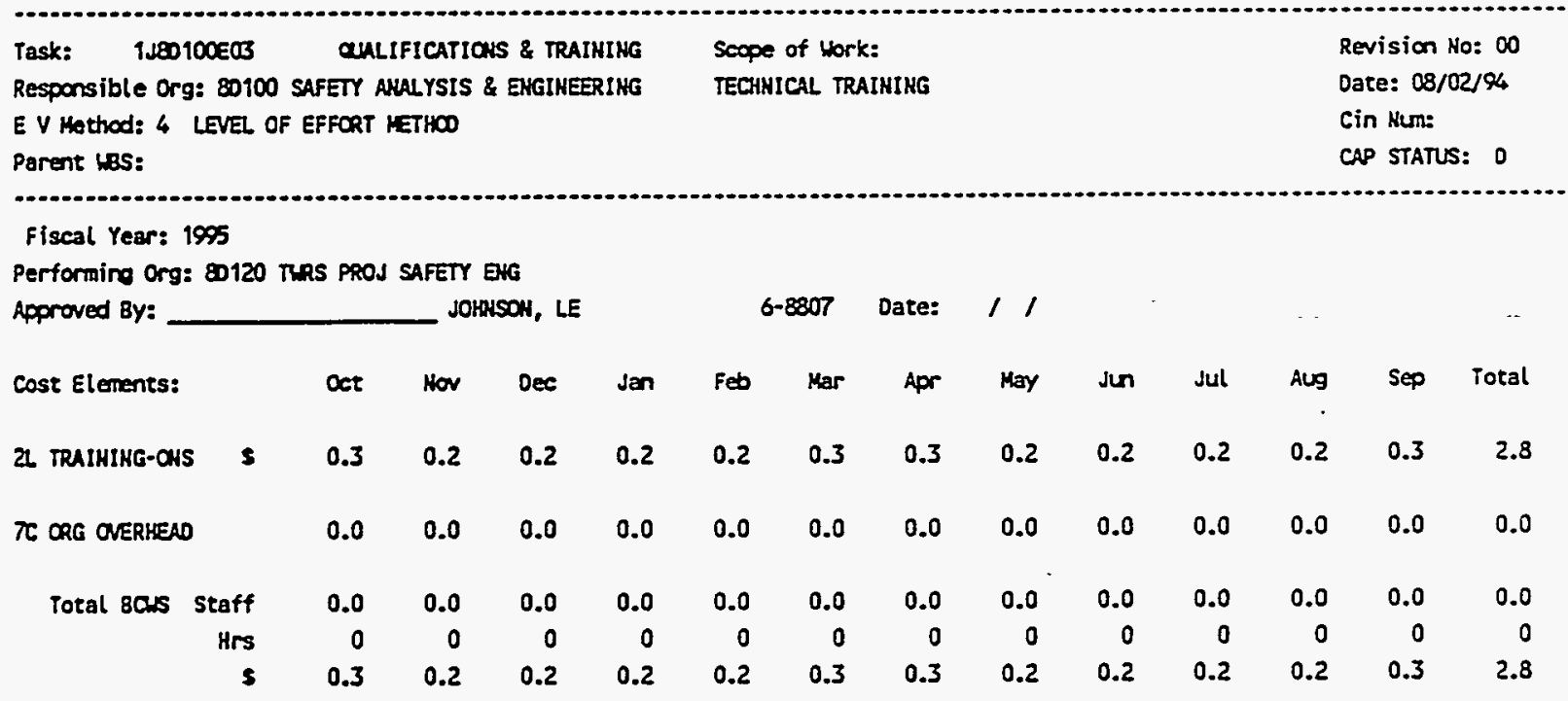


FOS-8620-R

Total BCP:

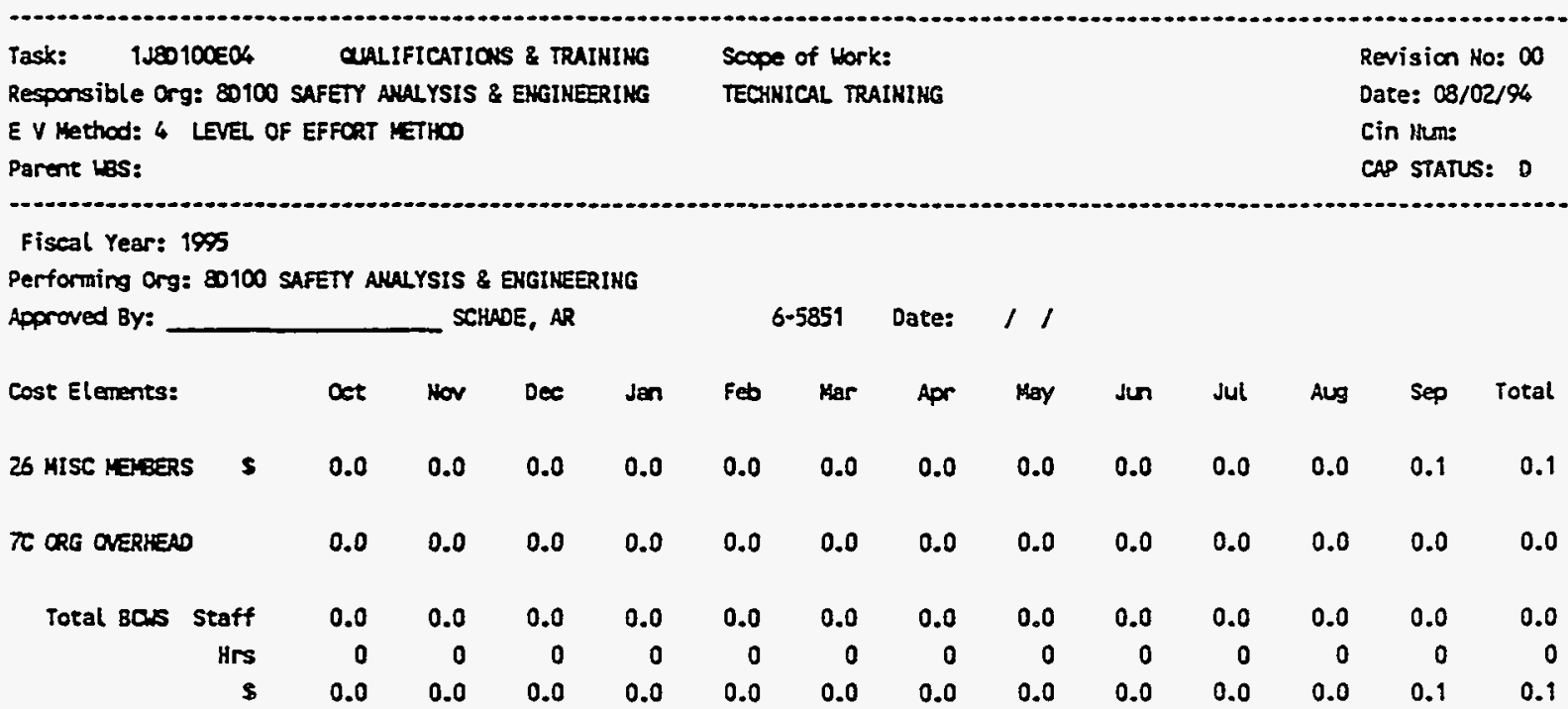

Total 80p:

Task: IJAD100EOS CUALIFICATIOUS \& TRAIHIHG

Responsible Org: 80100 SAFETY AUALYSIS \& EMGINEERING

E V Hethod: 4 LEVEL OF EFFORT HETHCO

Parent IBS:
Scope of Work:

TECHNICAL TRAINING
Revision Ho: 00 Date: $08 / 02 / 94$

Cin Hum: CAP STATUS: D

Fiscal Year: 1995

Performing Org: D0140 OPS TRAHS \& EN SAFETY ENG

Approved By: ZIMER, JJ

$$
\text { 6-2574 Date: } / 1
$$

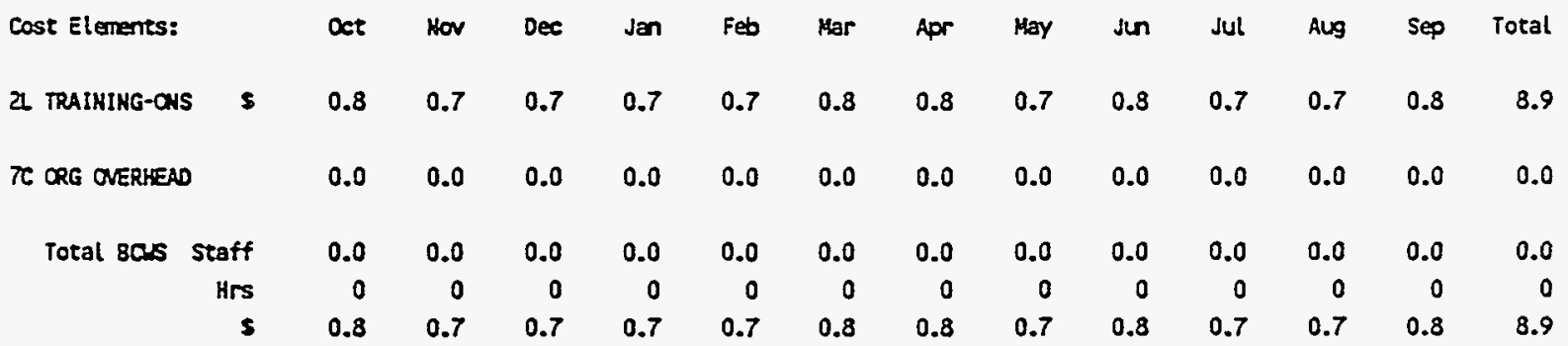

Total BCD:

Task: 1J80100EOS CUALIFICATIONS \& TRAINING Responsible Org: 80100 SAFETY AWHLYSIS \& ENGINEERING E V Wethod: 4 LEVEL OF EFFORT METHCO Parent lBs:

\section{Scope of Hork:} TECHMICAL TRAINING
Revision No: 00 Date: $08 / 02 / \%$

Cin Nus: cap status: 0

Fiscal Year: 1995 


\section{WHC-SP-1116 REV 0}

FDS-BG20-R

Performing Org: 80150 CRIT \& RADIOLOGICAL ANLLYSIS

Approved By: DAUETTRY, N

\begin{tabular}{|c|c|c|c|c|c|c|c|c|c|c|c|c|c|c|}
\hline Cost Elements: & & $\operatorname{cct}$ & Nov & Dec & Jan & Feb & Mar & Apr & May & $\operatorname{Jun}$ & Jut & Aug & Sep & Total \\
\hline 21. TRAIHIKG-ONS & $s$ & 0.5 & 0.5 & 0.5 & 0.5 & 0.5 & 0.5 & 0.5 & 0.5 & 0.5 & 0.5 & 0.5 & 0.6 & 6.1 \\
\hline$\pi$ CRG OVERKEAD & & 0.0 & 0.0 & 0.0 & 0.0 & 0.0 & 0.0 & 0.0 & 0.0 & 0.0 & 0.0 & 0.0 & 0.0 & 0 \\
\hline Total Bcus s & Staff & 0.0 & 0.0 & 0.0 & 0.0 & 0.0 & 0.0 & 0.0 & 0.0 & 0.0 & 0.0 & 0.0 & 0.0 & 0 \\
\hline & Hrs & 0 & 0 & 0 & 0 & 0 & 0 & 0 & 0 & 0 & 0 & 0 & 0 & 0 \\
\hline & s & 0.5 & 0.5 & 0.5 & 0.5 & 0.5 & 0.5 & 0.5 & 0.5 & 0.5 & 0.5 & 0.5 & 0.6 & \\
\hline
\end{tabular}

Total 8c:P:

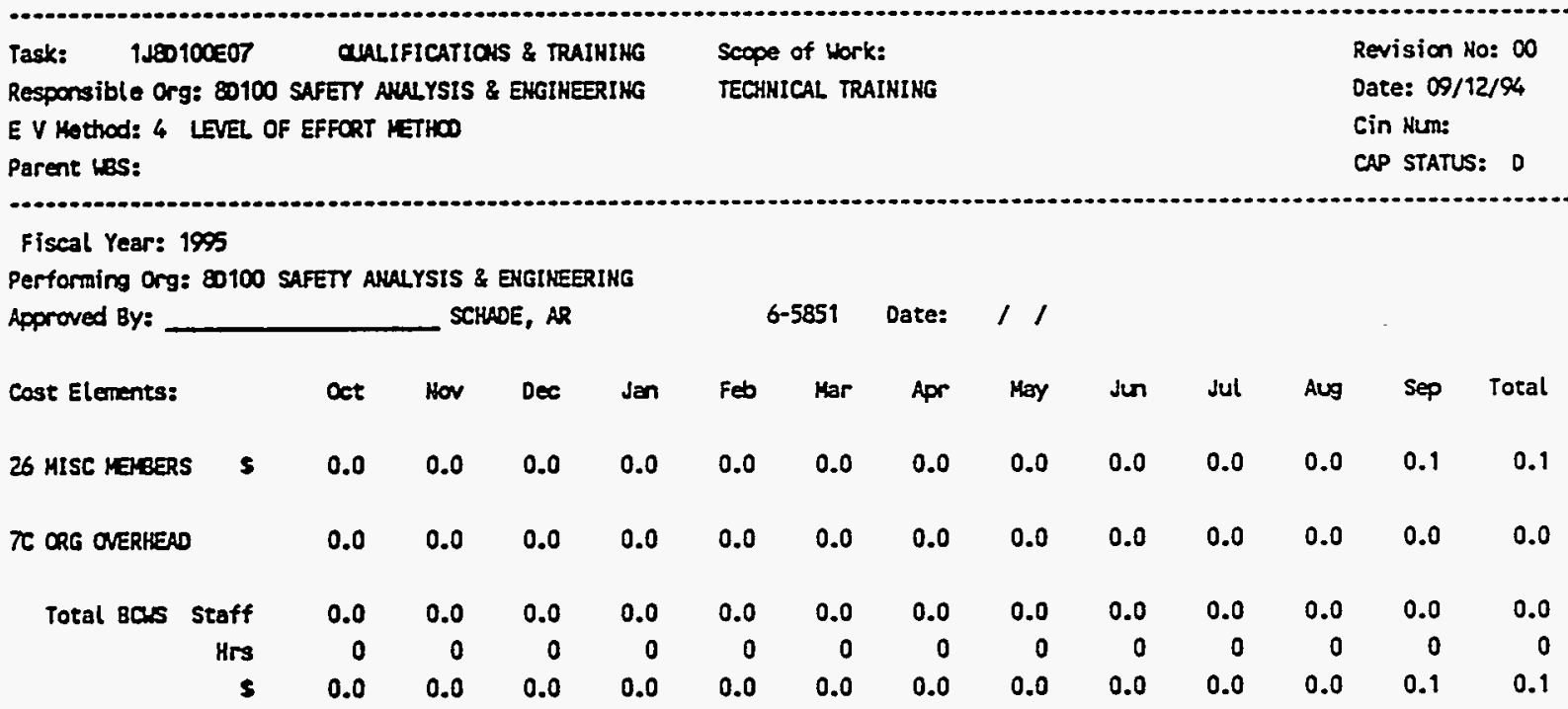

Total BCP:

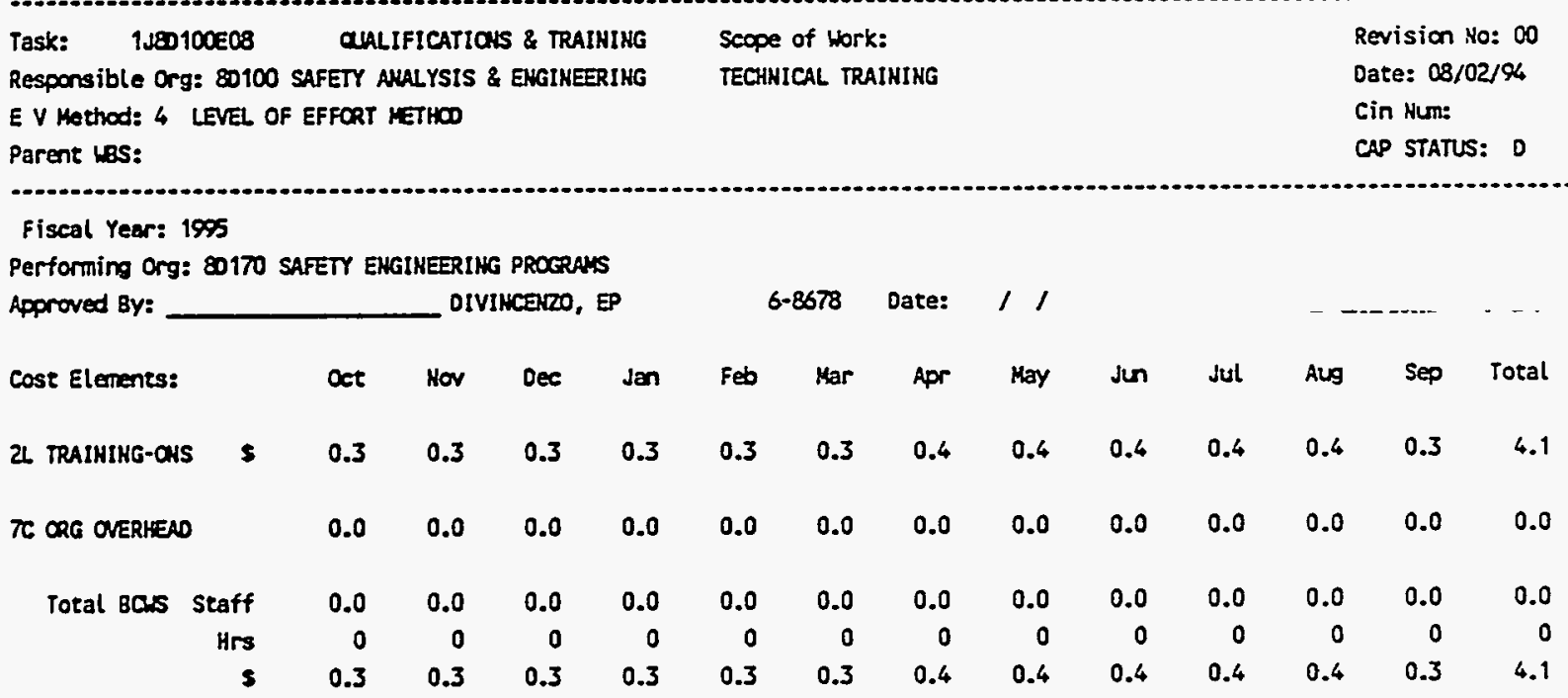

Total BCP: 


\section{WHC-SP-1116 REV 0}

FOS-B620-R

\begin{tabular}{|c|c|c|c|c|c|c|c|c|c|c|c|c|c|c|}
\hline Total ip & & Oet & Hor & Dec & Jan & Feb & Mar & Apr & May & Jun & Jul & Aug & 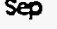 & al \\
\hline Z S UDIST BOGE & $\mathbf{s}$ & 0.0 & 1.0 & 0.0 & 0.0 & 1.0 & 0.0 & 0.0 & 0.0 & 1.0 & 0.0 & 0.0 & 0.0 & 3.0 \\
\hline 21 TRAINING-ONS & $\mathbf{s}$ & 3.3 & 3.3 & 3.2 & 3.3 & 3.2 & 3.5 & 3.5 & 3.4 & 3.4 & 3.4 & 3.6 & 3.7 & 40.6 \\
\hline 26 MISC MEVERS & $\mathbf{s}$ & 0.0 & 0.0 & 0.0 & 0.0 & 0.0 & 0.0 & 0.0 & 0.0 & 0.0 & 0.0 & 0.0 & 0.2 & 0.2 \\
\hline 41 TRKG-SAFEREH & $\mathbf{s}$ & 0.2 & 0.2 & 0.2 & 0.2 & 0.2 & 0.2 & 0.2 & 0.2 & 0.2 & 0.2 & 0.2 & 0.2 & 2.4 \\
\hline 46 OTRC TRAINIH & $\mathbf{s}$ & 0.2 & 0.2 & 0.2 & 0.2 & 0.2 & 0.2 & 0.2 & 0.2 & 0.2 & 0.2 & 0.2 & 0.3 & 2.5 \\
\hline$\pi$ ORG OVERHEAD & & 0.0 & 0.0 & 0.0 & 0.0 & 0.0 & 0.0 & 0.0 & 0.0 & 0.0 & 0.0 & 0.0 & 0.0 & 0.0 \\
\hline Total BCS S & Staff & 0.0 & 0.0 & 0.0 & 0.0 & 0.0 & 0.0 & 0.0 & 0.0 & 0.0 & 0.0 & 0.0 & 0.0 & 0.0 \\
\hline & Hrs & 0 & 0 & 0 & 0 & 0 & 0 & 0 & 0 & 0 & 0 & 0 & 0 & 0 \\
\hline & $\$$ & 3.7 & 4.7 & 3.6 & 3.7 & 4.6 & 3.9 & 3.9 & 3.8 & 4.8 & 3.8 & 3.8 & 4.8 & 48.7 \\
\hline
\end{tabular}

LORK PACCACE: IJ8O1OOF SA METHOOCOOGY \& STANDARDS SCOPE of HOrk: Resp Org:
Q100 SAFETY ANALYSIS \& ENGINEERING
Revision No: 00 Date: 08/02/94

CAP STATUS: D

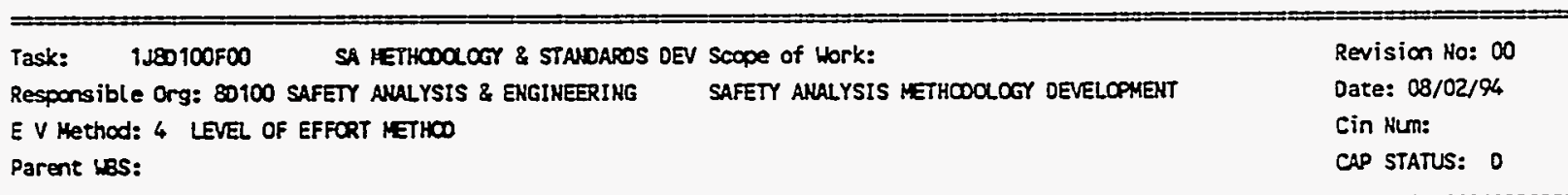

Milestones: Marrative

SAE- 5 -020 R 09/29/\%5 EXTERULL PEER REVIEN OF THE EXO CODE

SAE-95-021 R O6/30/95 IMPROVED ATMOSPHERIC OISPERSIOH MCDELS

SAE-95-022 R O6/30/95 DEVELOP OR OBTAIN METHODOOOGY FOR CHEMICAL MIXTRRE RELEASE ANALYSIS

SAE-95-023 R 06/30/\%5 DEVELCO A MDOEL TO CALCULATE RELEASES FROM A LIOUID POOL (SPILLS)

SAE-\$5-024 R 09/29/95 RELEASE FRACTICH/COMPUTER CODE ACTIVITIES

SAE-\$5-025 K 09/29/95 SAFETY ANALYSIS \& ENGINEERIMG METHOOQOOY DEVELOPMENT

Fiscal Year: 195

Performin Org: 80100 SAFETY AMALYSIS \& ENGINEERIHG

Approved By: SCHADE, AR

6-5851 Date: $/ 1$

\begin{tabular}{|c|c|c|c|c|c|c|c|c|c|c|c|c|c|c|}
\hline Cost Elements: & & $\infty e t$ & Hov & Dec & Jan & Feb & Mar & Apr & May & Jus & Jul & $A \cup g$ & Sep & Total \\
\hline 26 MISC MEMBERS & 5 & 0.0 & 0.0 & 0.0 & 0.0 & 0.0 & 0.0 & 0.0 & 0.0 & 0.0 & 0.0 & 0.0 & 0.1 & 0.1 \\
\hline$\pi$ ORG OVERHEAD & & 0.0 & 0.0 & 0.0 & 0.0 & 0.0 & 0.0 & 0.0 & 0.0 & 0.0 & 0.0 & 0.0 & 0.0 & 0.0 \\
\hline Total BCus & aff & 0.0 & 0.0 & 0.0 & 0.0 & 0.0 & 0.0 & 0.0 & 0.0 & 0.0 & 0.0 & 0.0 & 0.0 & 0.0 \\
\hline
\end{tabular}




\section{WHC-SP-1116 REV 0}

FDS-B620-R

Financial Date System

COST ACCONNT PLAN - 1J8010 - VERSICN 1995

$09 / 14 / 94,14: 22$

Page: 17

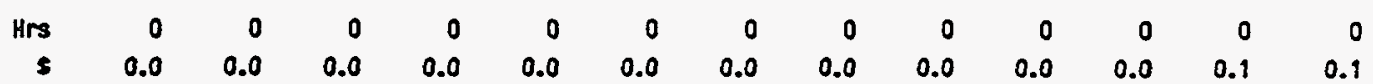

Total BOP:

Task: 1J80100F01 SA NETHCOOCOOY \& STADAADS DEV SCOpe of Hork:

Responsible Org: 80100 SAFETY ANALYSIS \& ENGIHEERING - SAFETY AKALYSIS STANDAROS DEVELOPYENT

E V Hethod: 4 LEVEL OF EFFCRT FETHOD

Parent LES:
Revision No: $\infty$ Date: $08 / 02 / 94$ Cin Kum:

CAP STATUS: 0

Fiscal Year: 1995

Performing Org: 80100 SAFETY AHLLYSIS \& ENGINEERIKG

Approved By: SCHADE, AR

6-5859 Date: / /

\begin{tabular}{|c|c|c|c|c|c|c|c|c|c|c|c|c|c|c|}
\hline Cost Elements: & & cet & Hor & Dec & Jen & Feb & Mar & Apr & May & Jun & Jul & Aug & $\operatorname{sep}$ & Total \\
\hline 26 MISC MEMBERS & s & 0.0 & 0.0 & 0.0 & 0.0 & 0.0 & 0.0 & 0.0 & 0.0 & 0.0 & 0.0 & 0.0 & 0.1 & 0.1 \\
\hline$\pi$ QRG OVERHEAD & & 0.0 & 0.0 & 0.0 & 0.0 & 0.0 & 0.0 & 0.0 & 0.0 & 0.0 & 0.0 & 0.0 & 0.0 & .0 \\
\hline Total BCus & Staff & 0.0 & 0.0 & 0.0 & 0.0 & 0.0 & 0.0 & 0.0 & 0.0 & 0.0 & 0.0 & 0.0 & 0.0 & \\
\hline & Hrs & 0 & 0 & 0 & 0 & 0 & 0 & 0 & 0 & 0 & 0 & 0 & 0 & 0 \\
\hline & $s$ & 0.0 & 0.0 & 0.0 & 0.0 & 0.0 & 0.0 & 0.0 & 0.0 & 0.0 & 0.0 & 0.0 & 0.1 & 0.1 \\
\hline
\end{tabular}

Total BCP:

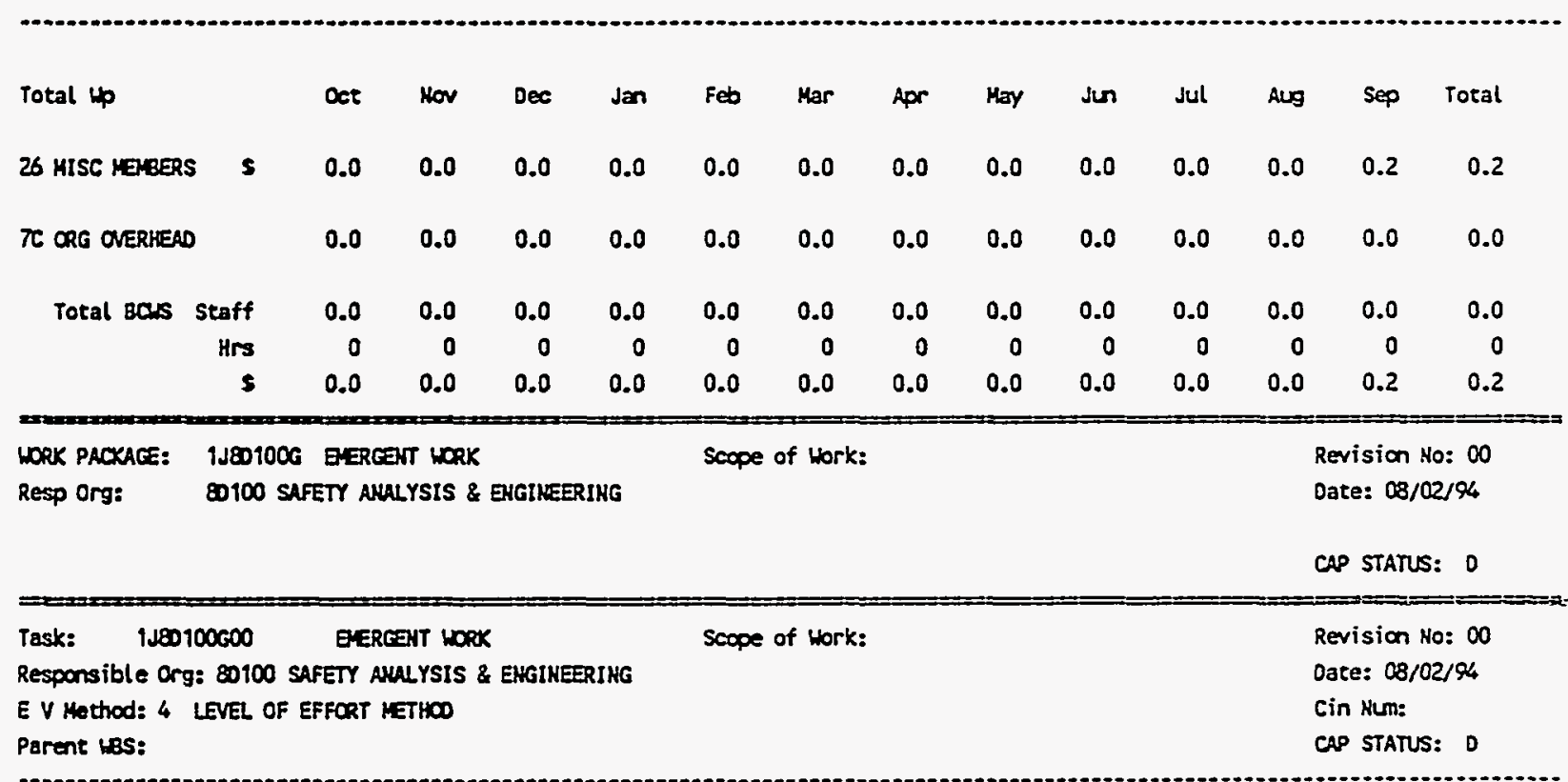

Fiseal Year: 1995

Performing Org: 80100 SAFETY AKALYSIS \& ENGINEERING

Approved By: SCHADE, AR

6-5851 Date: $/ /$

Cost Elements: Det Hov Dec Jan feb Mar Apr May Jun Jul Aug Sep Total

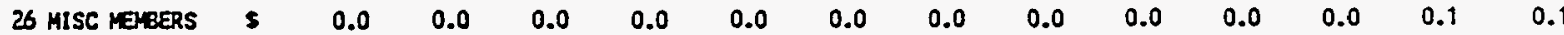


WHC-SP-1116 REV 0

FDS-8620-R

Financial Data System

09/14/94 14:22

COST ACCONT PLAN - 1J8010 - VERSION 1995

Page: 18

\begin{tabular}{|c|c|c|c|c|c|c|c|c|c|c|c|c|c|c|}
\hline C CRG OVERHEA & & 0.0 & 0.0 & 0.0 & 0.0 & 0.0 & 0.0 & 0.0 & 0.0 & 0.0 & 0.0 & 0.0 & 0.0 & 0.0 \\
\hline Total bous & Staff & 0.0 & 0.0 & 0.0 & 0.0 & 0.0 & 0.0 & 0.0 & 0.0 & 0.0 & 0.0 & 0.0 & 0.0 & 0 \\
\hline & Hrs & 0 & 0 & 0 & 0 & 0 & 0 & 0 & 0 & 0 & 0 & 0 & 0 & 0 \\
\hline & s & 0.0 & 0.0 & 0.0 & 0.0 & 0.0 & 0.0 & 0.0 & 0.0 & 0.0 & 0.0 & 0.0 & 0.1 & 0.1 \\
\hline
\end{tabular}

Total sap:

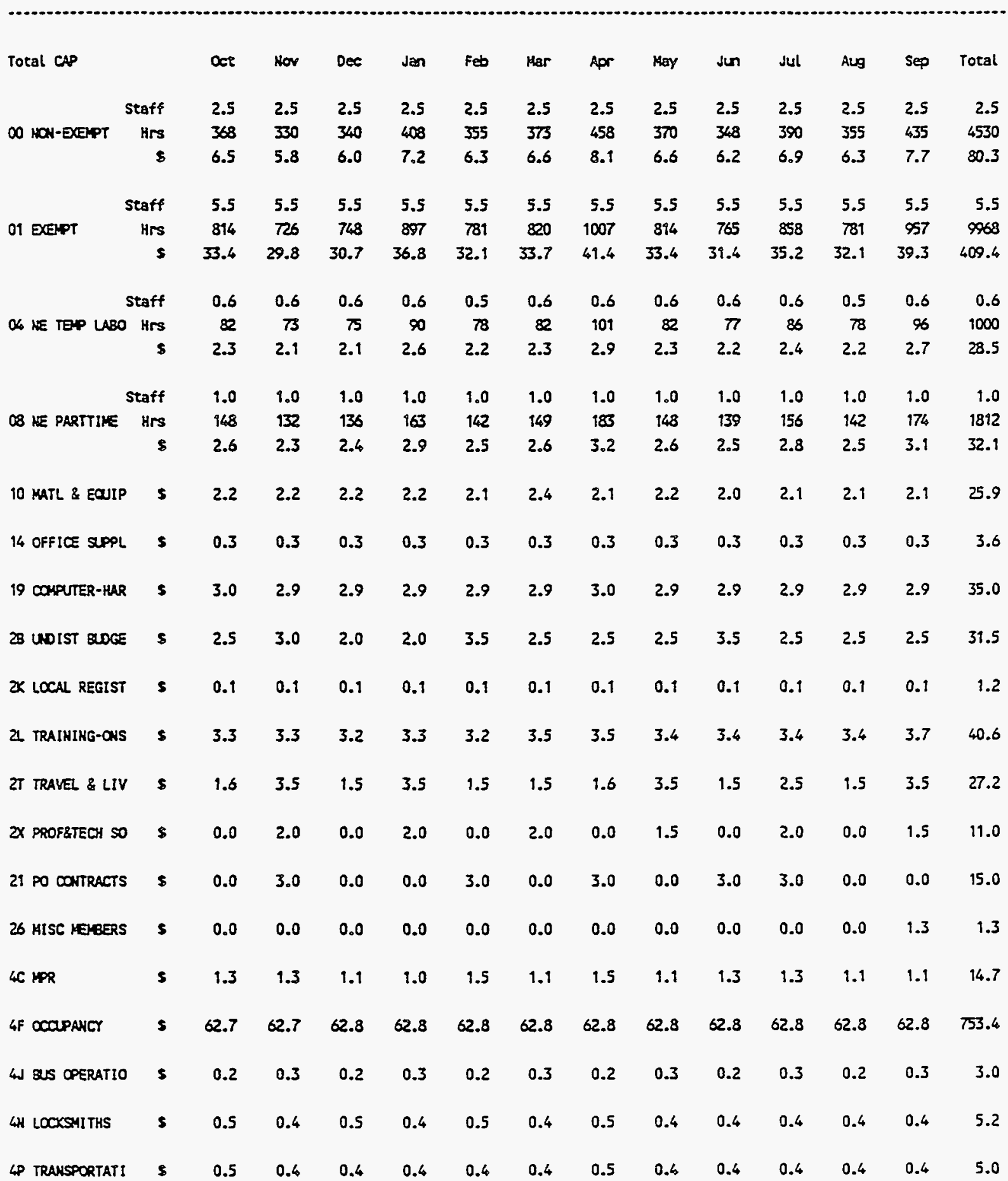




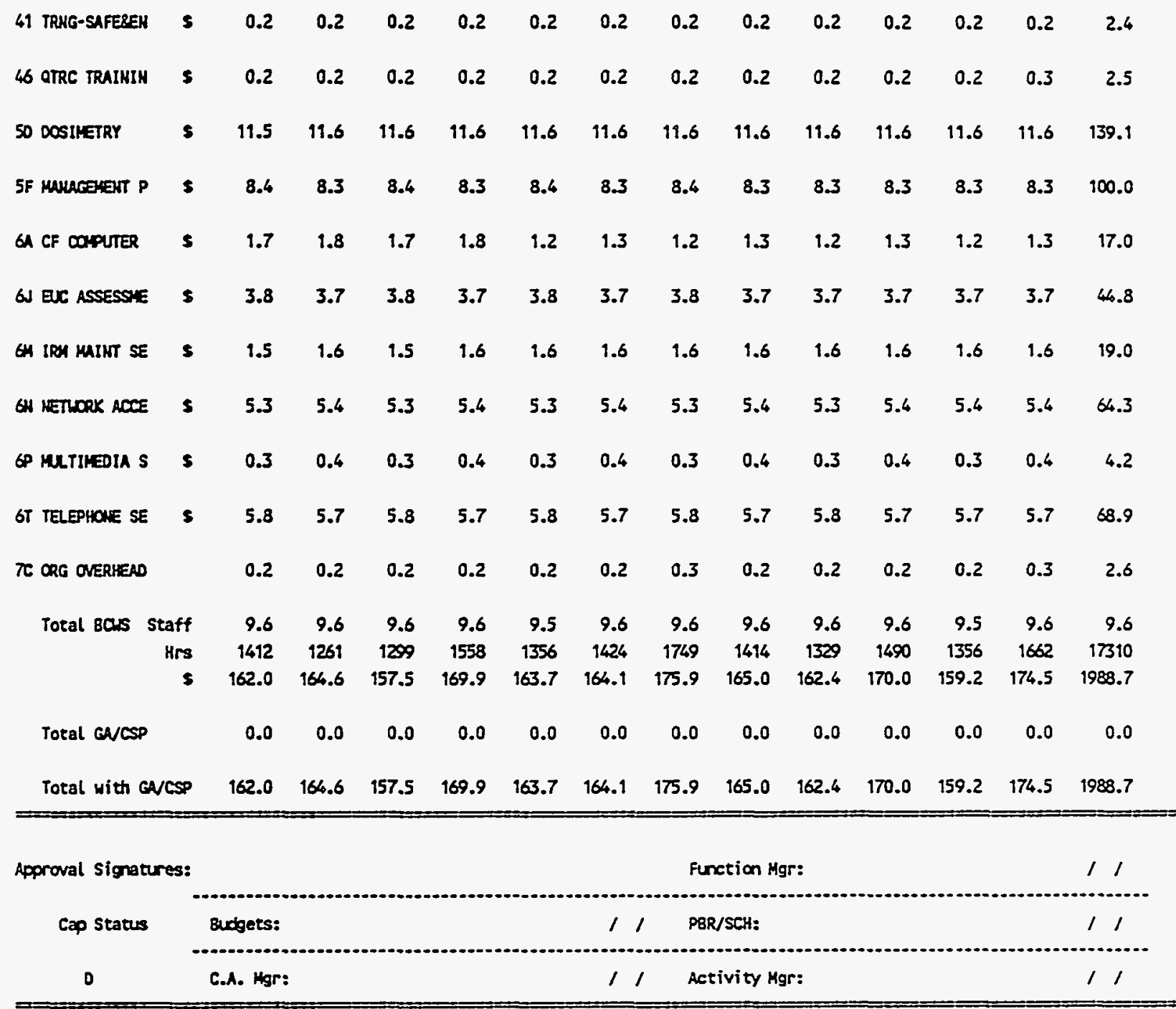


HHC-SP-1116 REV 0

Financial Data Systen

$09 / 14 / 94 \quad 14: 22$

FOS-8620-R

COST ACCONT PLAN - 1J8010 - VERSTON 1995

Page: 20

ED OF FOS8620

Overheads, Safety Analysis \& Engineering Page 3-50 
$1 \mathrm{~J} 80100800$ J801E

APPENDIX A

OVERHEADS, SAFETY AHALYSIS AHD EKGINEERIHG

PROGRAH DEVELOPMENT \& IMPLEMENTATIOH

MILESTOHE/UNIT OF MEASURE (UOM)

ESTIMATE 
$1 \mathrm{~J} 80100800$

J801E (continued)

\section{WHC-SP.-1116 REV 0}

APPENDIX A

OVERHEADS, SAFETY AKALYSIS AND ENGINEERIHG

PROGRAH DEVELOPMENT \& IMPLEMENTATIOH

HILESTONE/UNIT OF MEASURE (UOA)

ESTIMATE

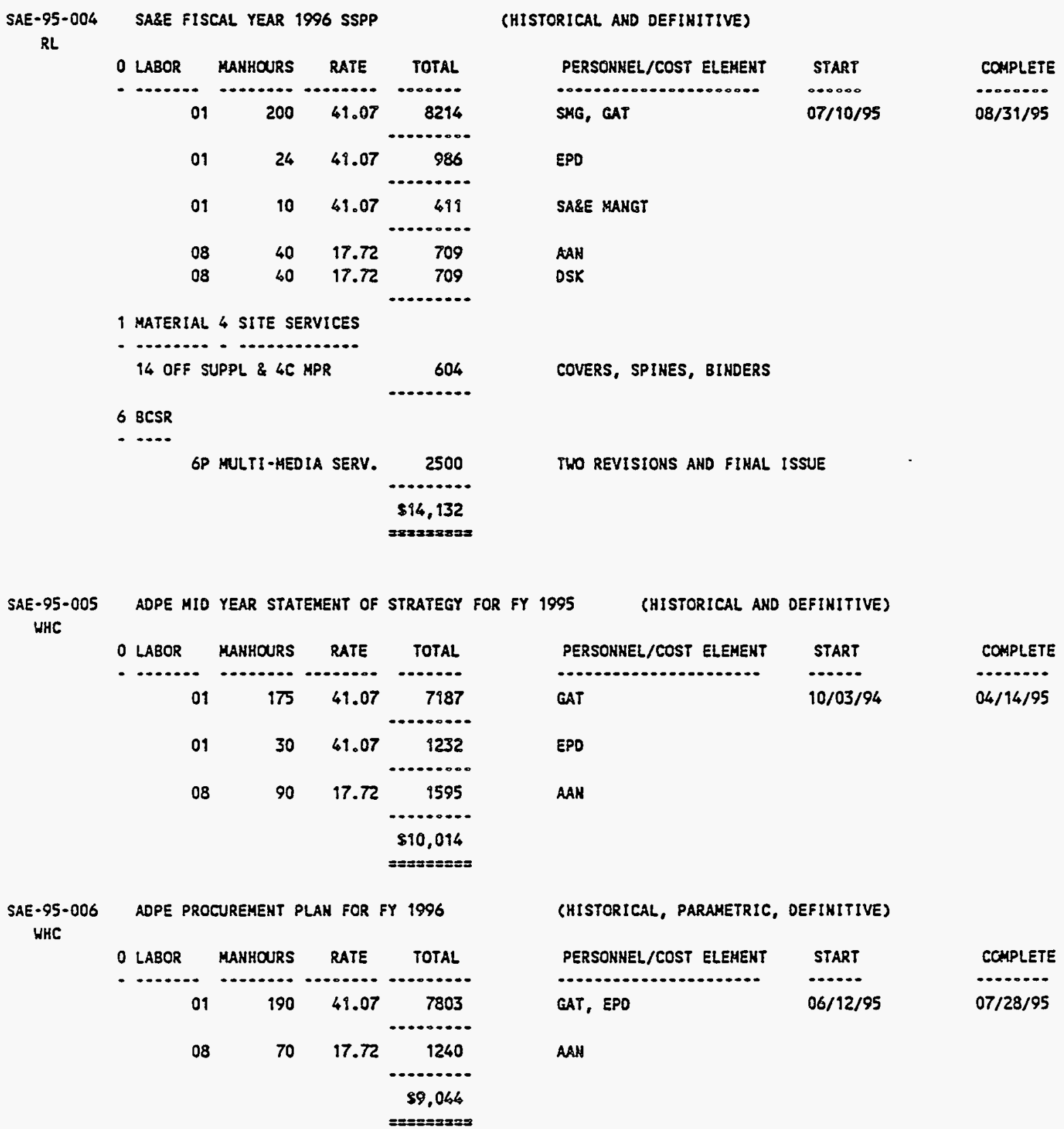

SAE-95-004 SA\&E FISCAL YEAR 1996 SSPP

RL

\begin{tabular}{crrrr}
0 LABOR & MAHHOURS & RATE & TOTAL \\
\hline 01 & 200 & 41.07 & 8214 \\
01 & 24 & 49.07 & 986 \\
01 & 10 & 49.07 & 419 \\
08 & 40 & 17.72 & 709 \\
08 & 40 & 17.72 & 709
\end{tabular}

1 MATERIAL 4 SITE SERVICES

- 14 OFF

14 OFF SUPPL \& $4 C$ MPR

604

6 BCSR

6P MULTI-MEDIA SERV.

2500

$\$ 14,132$

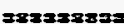

(HISTORICAL AND DEFINITIVE)

\begin{tabular}{|c|c|}
\hline ONHEL/COST ELEKENT & START \\
\hline SMG, GAT & $07 / 90 / 95$ \\
\hline
\end{tabular}

EPD

SAEE MANGT

AAN

DSK

COVERS, SPIHES, BIHDERS

TWO REVISIONS AND FIHAL ISSUE

SAE-95-005 ADPE MIO YEAR STATEMENT OF STRATEGY FOR FY 1995 (HISTORICAL AND DEFIHITIVE) WHC

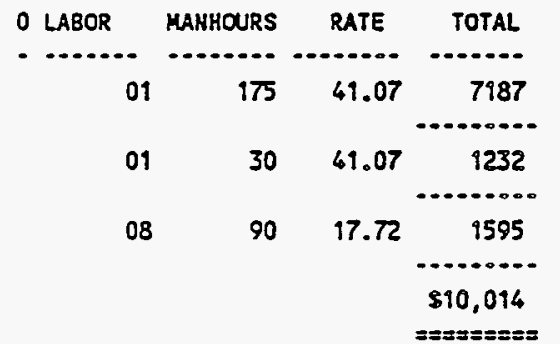

SAE-95-006 ADPE PROCUREMENT PLAM FOR FY 1996 UHC

\begin{tabular}{|c|c|c|}
\hline PERSONWEL/COST ELEMENT & START & COMPLETE \\
\hline$\cdots$ & $\ldots$ & $\cdots$ \\
\hline GAT & $10 / 03 / 94$ & $04 / 1$ \\
\hline
\end{tabular}

EPD

AAH

(HISTORICAL, PARAMETRIC, DEFINITIVE)

\begin{tabular}{lll} 
PERSONMEL/COST ELEMENT & START & CCMPLETE \\
\hdashline GAT, EPO & $06 / 12 / 95$ & $07 / 28 / 95$
\end{tabular}

AAM

$\$ 9.044$

$=====2353$ 
$1 \mathrm{~J} 80100800$

J801E (continued)

\section{WHC-SP-1116 REV 0}

APPENDIX A

OVERHEADS, SAFETY AMALYSIS AND EHGIMEERING

PROGRAY DEVELOPMENT \& IMPLEMENTATIOH

MILESTOHE/UHIT OF MEASURE (UOH)

ESTIMATE

SAE-95-007 SARE MONTHLY PERFORMAHCE STATISTICS WHC

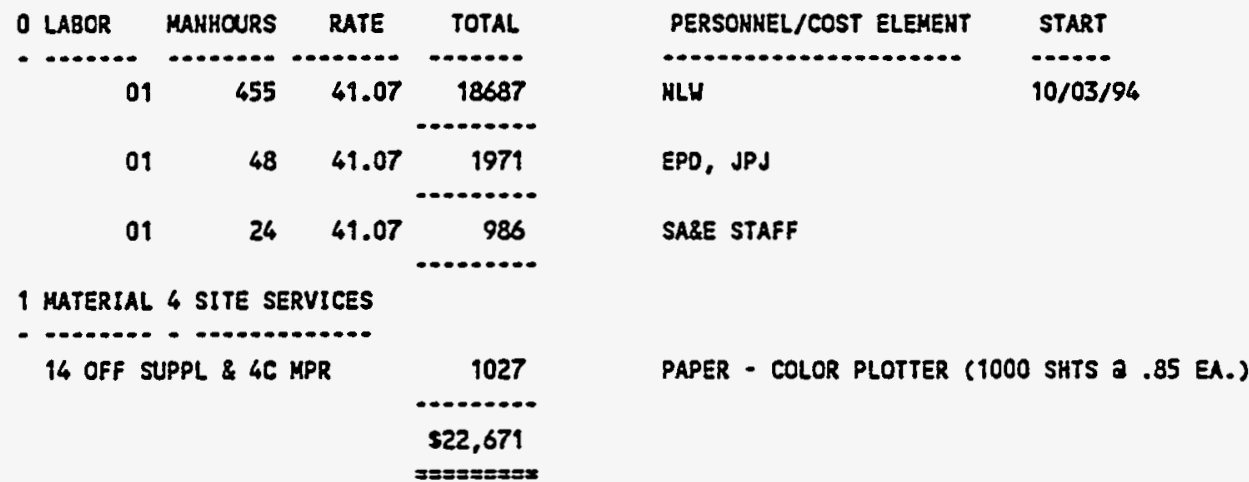

SAE-095-008 SA\&E SITE MAHAGEMENT SYSTEM REPORT (HISTORICAL AND DEFINITIVE) WHC

\begin{tabular}{|c|c|c|c|}
\hline O LABOR & MANHOURS & RATE & TOTAL \\
\hline . & $\ldots+\ldots$ & $\ldots$ & \\
\hline 01 & 240 & 49.07 & 9857 \\
\hline 01 & 136 & 49.07 & 5586 \\
\hline 08 & 48 & 17.72 & 851 \\
\hline
\end{tabular}

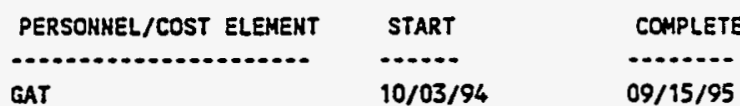

EPD, SHE

AAN
COMPLETE

$09 / 15 / 95$

SAE- $095-009$

SABE SITE VARLANCE AHD CORRECTIVE ACTIOH REPORT

(HISTORICAL AKD DEFINITIVE) WHC

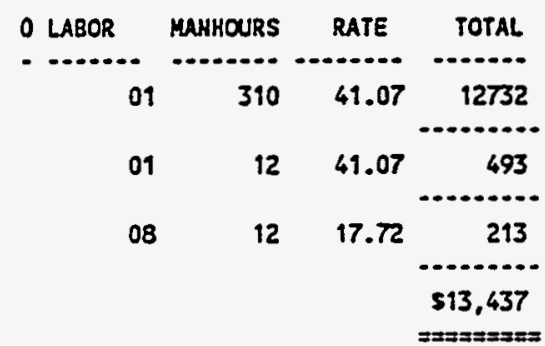

PERSOHNEL/COST ELEMENT

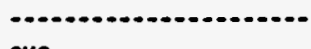

SHG

START

-......

$10 / 03 / 94$

EPD

AAN
COMPLETE

can......

$09 / 15 / 95$

Overheads, Safety Analysis \& Engineering Page 4-3 
1 1580100800

J801E (continued)

\section{WHC-SP-1116 REV 0}

APPENDIX A

OVERHEADS, SAFETY AMALYSIS AND ENGIMEERING

PROGRAM DEVELOPHENT \& IMPLEMENTATIOH

MILESTONE/UNIT OF MEASURE (UOH)

ESTIMATE

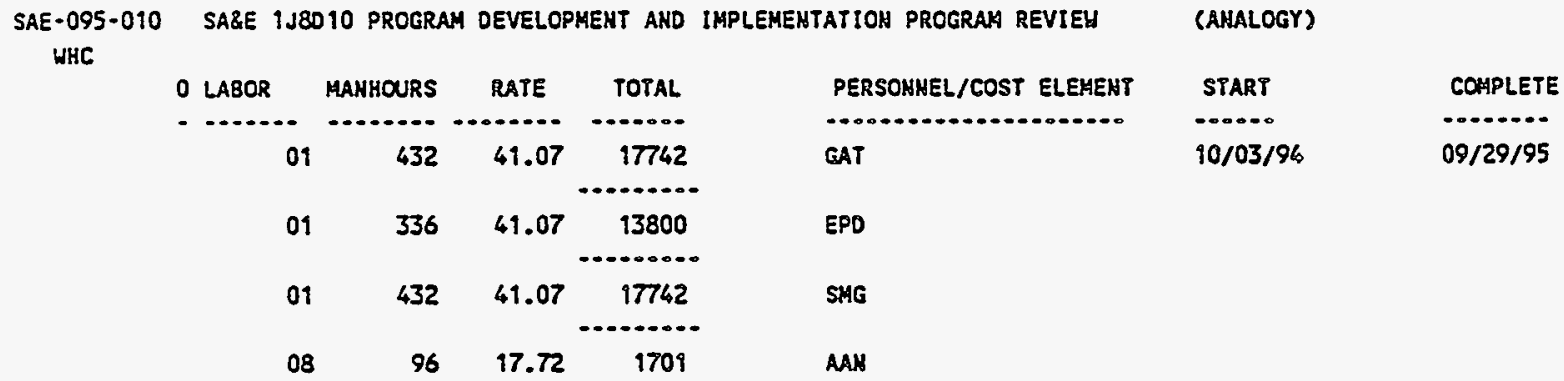

\begin{tabular}{|c|c|c|c|c|c|c|}
\hline \multirow{3}{*}{$\begin{array}{l}0 \text { LABOR } \\
01 \\
01\end{array}$} & MAHHOURS & RATE & TOTAL & PERSONHEL/COST ELEMEKT & START & COMPLETE \\
\hline & n...... & $\ldots \ldots$ & -..... & (n) & $\cdots \ldots$ & ......... \\
\hline & 24 & 49.07 & 986 & GAT & $01 / 03 / 95$ & $04 / 14 / 95$ \\
\hline 01 & 12 & 41.07 & 693 & SHG & & \\
\hline 01 & 1.5 & 41.07 & 62 & EPD & & \\
\hline 08 & 3 & 17.72 & $\begin{array}{r}53 \\
\$ 1,593 \\
= \pm 7=x=28\end{array}$ & AAN & & \\
\hline
\end{tabular}

(DEFINITIVE)

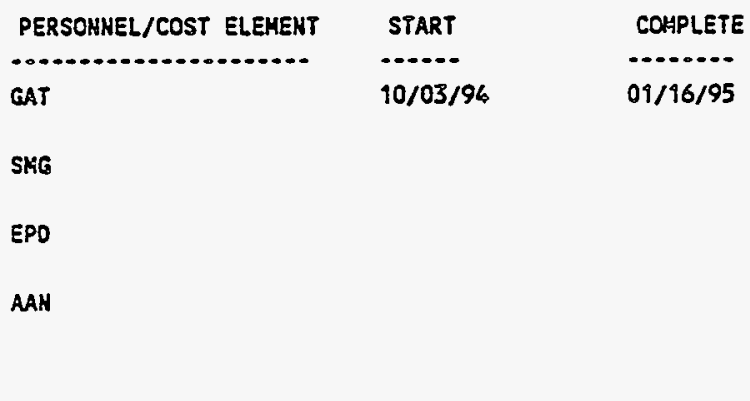

(DEFINITIVE)

$==x+2 x=2 x$ 
is 80100800

J801E (cont inued)
WHC-SP-1116 REV 0

APPEKDIX A

OVERHEADS, SAFETY AKALYSIS AHD ENGIMEERING

PROGRAM DEVELOPMENT \& IMPLEMEMTATION

MILESTOME/UHIT OF MEASURE (UOH)

ESTIMATE
SAE-095-013 QUARTERLY PROGRESS REPORT OF SARE

RL

\begin{tabular}{|c|c|c|c|}
\hline $\begin{array}{l}0 \text { LABOR } \\
01\end{array}$ & MAHKOURS & $\begin{array}{l}\text { RATE } \\
41.07\end{array}$ & $\begin{array}{r}\text { TOTAL } \\
\ldots . . . \\
986\end{array}$ \\
\hline 01 & 12 & 41.07 & 493 \\
\hline 01 & 1.5 & 49.07 & 62 \\
\hline 08 & 3 & 17.72 & $\begin{array}{r}53 \\
51,593 \\
=2=2=2=x=\end{array}$ \\
\hline
\end{tabular}

SAE-095-014 QUARTERLY PROGRESS REPORT OF SABE

RL

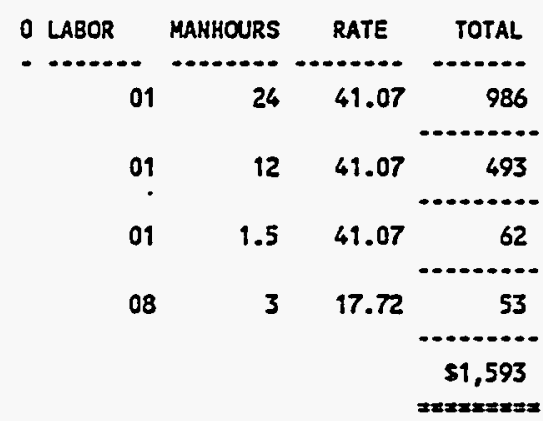

(DEFIMITIVE)

\begin{tabular}{|c|c|c|}
\hline PERSONHEL/COST ELEMENT & START & COMPLETE \\
\hline GAT & $04 / 03 / 95$ & $07 / 14 / 95$ \\
\hline
\end{tabular}

SHG

EPD

AAH

(DEFINITIVE)

PERSONMEL/COST ELEMENT
GAT
SHG
EPO
AAM

START

COMPLETE

$07105 / 95$

-........

$07 / 05 / 95 \quad 09 / 29 / 95$ 
$1 \mathrm{~J} 80100800$

J801U
WHC-SP-1116 REV 0

APPENDIX A

OVERHEADS, SAFETY AMALYSIS AKD ENGINEERIMG

MANDATORY PLAHHING

MILESTONE/UHIT OF MEASURE (UOM) ESTIMATE

SAE-095-015 SAFETY AMALYSIS \& ENGINEERIHG ANNUAL UPOATE OF TRAINING RECORDS HHC

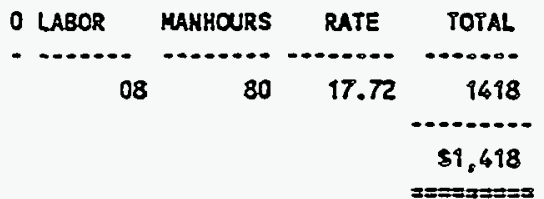

PERSOHAEL/COST ELEMENT

(DEFIHITIVE)

S4I

START

COMPLETE

$08109 / 95$

$08 / 31 / 95$ 
1180100804

Ј801J
HHC-SP-1116 REV 0

APPENDIX A

OVERHEADS, SAFETY AHALYSIS AND ENGIHEERIHG

COHTRACTS ADMIHISTRATIOH

MILESTONE/UHIT OF MEASURE (UOM)

ESTIMATE

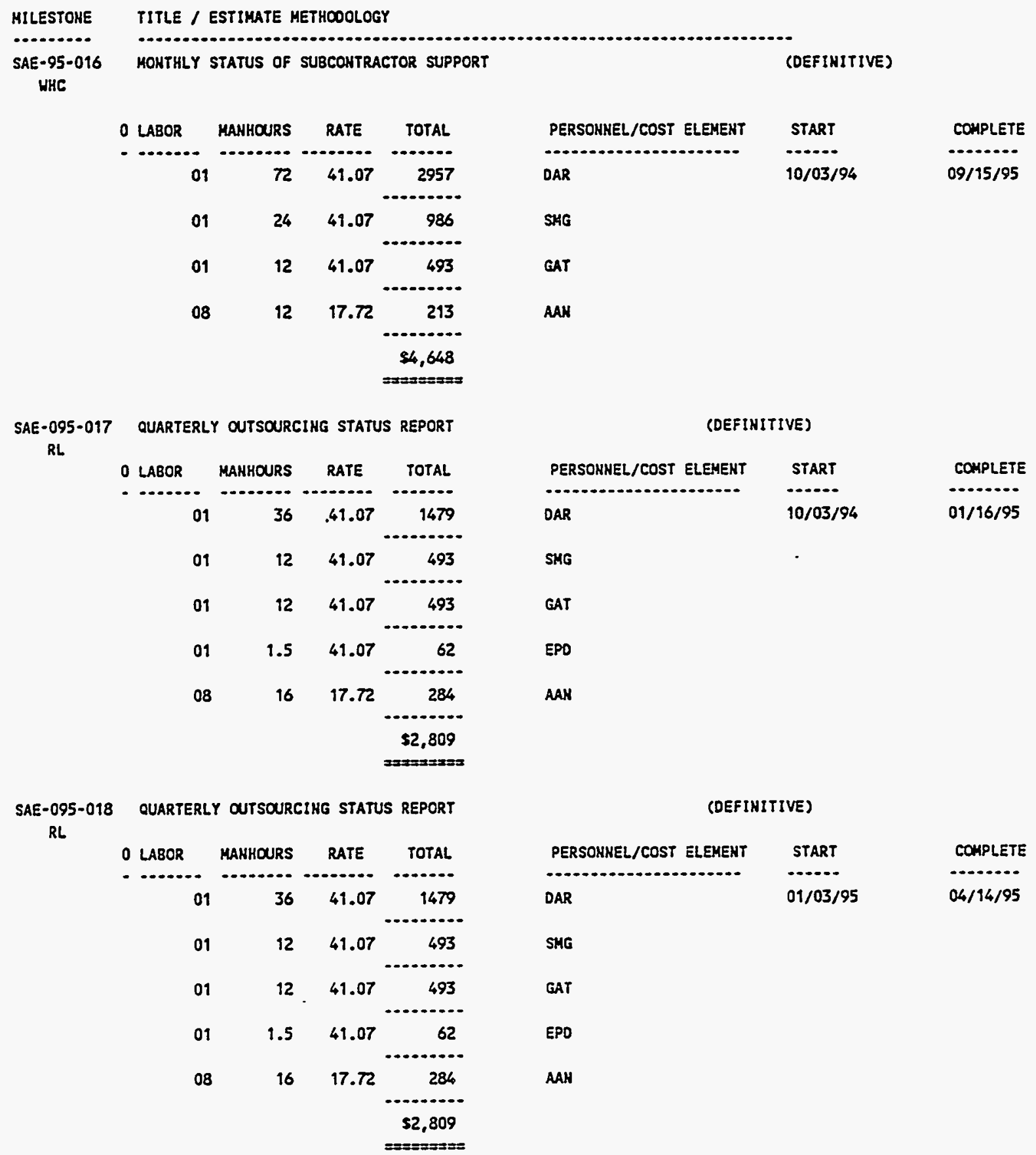


1380100804

J801/
WHC-SP-1116 REV 0

APPENDIX A

OVERHEADS, SAFETY AKALYSIS AND ENGIMEERING CONTRACTS ADHINISTRATIOH

MILESTONE/UNIT OF MEASURE (UOA)
SAE-095-019 QUARTERLY OUTSOURCING STATUS REPORT

RL
(DEFIHITIVE)

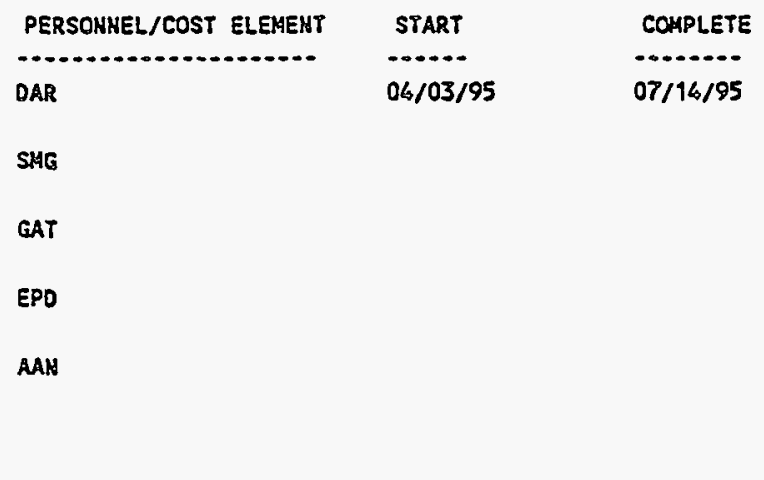

(DEFINITIVE)

\begin{tabular}{|c|c|c|}
\hline PERSONKEL/COST ELEMENT & START & COMPLETE \\
\hline - & $\cdots$ & $\cdots \cdots$ \\
\hline DAR & $07 / 05 / 95$ & $09 / 29 / 95$ \\
\hline
\end{tabular}

SHG

GAT

EPD

AAN
RL

\begin{tabular}{|c|c|c|c|}
\hline 0 LABCR & MANHOURS & RATE & TOTAL \\
\hline - & - & $\cdots$ & -...... \\
\hline 09 & 36 & 49.07 & 1479 \\
\hline 09 & 12 & 41.07 & 493 \\
\hline 01 & 12 & 49.07 & 493 \\
\hline 09 & 1.5 & 41.07 & 62 \\
\hline 08 & 16 & 17.72 & 286 \\
\hline & & & $\begin{array}{r}\$ 2,809 \\
=2=x=x=0\end{array}$ \\
\hline
\end{tabular}




\section{WHC-SP-1116 REV 0}

$1 / 80100800$ J801E
APPENDIX $A$

OVERHEADS, SAFETY ANALYSIS AND ENGINEERIHG

PROGRAM DEVELOPHEHT \& IMPLEMENTATIOH

MILESTOHE/UHIT OF MEASURE (UOH)

ESTIMATE

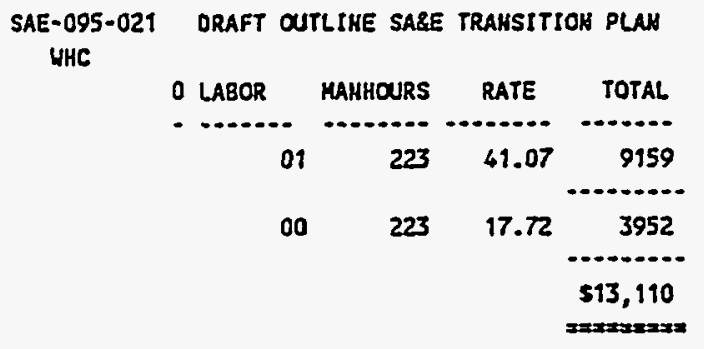

SAE-095-022 TRAHSITION PLAK

$R L$
(DEFIHITIVE)

\begin{tabular}{lll} 
PERSONHEL/COST ELEKENT & START & COMPLETE \\
\hdashline JJZ & $-10 / 01 / 94$ & $11 / 15 / 94$
\end{tabular}

PEX

(DEFIHITIVE)

\begin{tabular}{lll} 
PERSOMHEL/COST ELEMENT & START & COMPLETE \\
\hdashline $\mathrm{NJZ}$ & $11 / 16 / 94$ & $01 / 15 / 95$
\end{tabular}

PEK 
WHC-SP-1116 REV 0

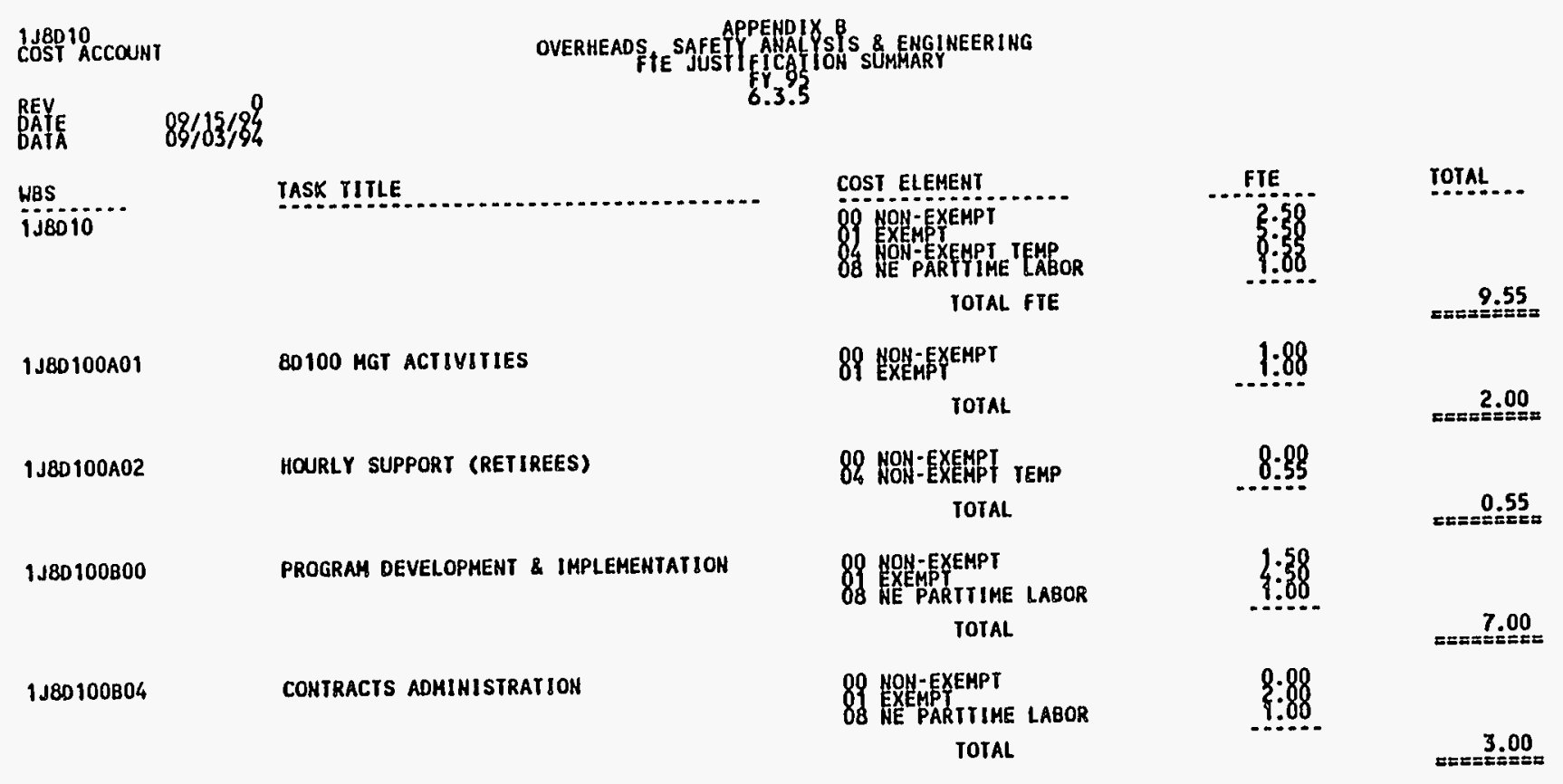




\section{WHC-SP-1116 REV 0}

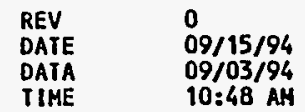

OVERHEADS, SAFETY ANALYSIS \& ENGIHEERING

FTE JUSTIFICATION

COST ELEMENT

HBS

TPCH TASK TITLE

FIE

J80100.- J8D MOH DISCAETIOMARY OPERATIMG COSTS

IJ80100A01 J801B 80100 HGT

$\begin{array}{lll}1 J 80100 A 03 & \text { J8D10 } & \text { STAFF MOVES } \\ 1 \text { J } 10001000 & \text { JBDIE } & \text { PROGRAH DEVELOPHENT \& IMPLEMENTATIOH }\end{array}$

$1 J 80100802$ J801G EFCOG ACTIVITIES

1JBD100B03 J8DIH PROFESSIONAL SOCIETY

$1 \mathrm{~J} 8 \mathrm{D} 100 \mathrm{E} 00 \mathrm{~J} \mathrm{~J} 8 \mathrm{D} 1 \mathrm{H}$

1J80100E01 J8DIV 8D100 TECHNICAL TRAINING

$1 J 80100 E 02$ J8DIH 80110 TECHHICAL TRAINING

J8D100E03 J801Y 80120 TECINICAL TRAINING

IJ8D100E05 J8D 1 80140 TECHNICAL IRAINING

IJ80100E06 J8D 12 80150 TECHNICAL TRAINING

IJ8D 100600 J8017 EMERGEHT MORK

TOTALS 
WHC-SP-1116 REV 0

$\begin{array}{ll}\text { REV } & 0 \\ \text { DAIE } & 09 / 15 / 94 \\ \text { DAIA } & 09 / 03 / 94 \\ \text { TIME } & 10: 48 \text { AH }\end{array}$

HBS

TPCN TASK TITLE

$1180100 \mathrm{AOO}$ J801A

IJ80 100A01 J8018

J80 100AO2 J8DIC

iJ80100A03 J8D10

1 J 80100800 J8D1E

$1 J 80100802 \quad 58016$

IJ80100B03 J8D1K

$1 J 80100000$ J801U

J8D 100001 J8DIV

IJ80100E02 J8D IW

1 J8D 100E03 JBD1Y

1 J8D 100E05 J8D11

1 J8D 100E06 J8D12

$\begin{array}{ll}\text { iJ80100E08 } & \mathrm{J} 8014 \\ \text { iJ80 100G00 } & \mathrm{J} 8017\end{array}$

rotals

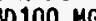

OVERHEADS, APPENDIX B

SAFETY AHALYSIS \& ENGINEERING

FIE JUSTIFICATION

$$
\begin{aligned}
& \text { ELEMENT } \\
& 6.3 .5
\end{aligned}
$$

fTE

MGT ACTIVITIES

HOURLY SUPPORT ( 48005 RETIREES)

EFCOG ACTIVITIES

PROFESSIONAL SOCIETY

CONTRACTS ADMIHISTRATIO

(ONDATINING

8D100 TECHNICAL TRAINING

8D110 TECHAICAL TRAIHING

BD 140 TECHNICAL TRAINING

80150 IECHNICAL TRAINING

8DI70 TECHNICAL TRAINING

EMERGENT WORK
1.00 SCHADE 1.0

2.50 DIVIHCENZO .75 , GOOFREY

50, TARZCA .75, ZIMMER .50

2.00 DIVIHCENZO .25, GODFREY

50, TARZCA .25, DAR 9.0

5.50

Overheads, Safety Analysis \& Engineering Page 4-12 


\section{WHC-SP-1116 REV 0}

$\begin{array}{ll}\text { REV } & 0 \\ \text { DATE } & 09 / 15 / 94 \\ \text { DATA } & 06 / 03 / 94 \\ \text { TIME } & 10: 48 \text { AN }\end{array}$

OVERHEADS, SAFEPE ANALIX B

$06 / 03394$
$10: 48$ AM

FTE JUSTIFICATION

COST ELEMENT 04

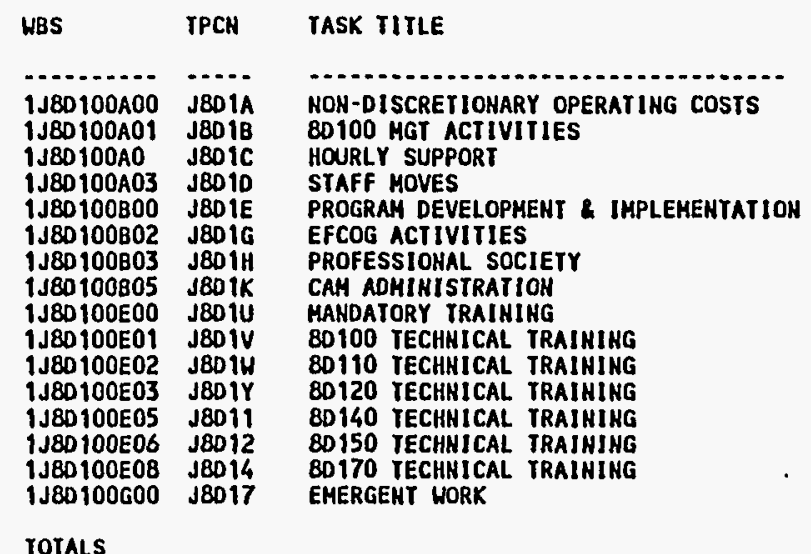

FTE

(..............

0.55

SEMHEROH

rotals 


\section{WHC-SP-1116 REV 0}

$\begin{array}{ll}\text { REV } & 0 \\ \text { DAIE } & 09 / 15 / 94 \\ \text { DAIA } & 06 / 03 / 94 \\ \text { IIME } & 10: 48 \text { AM }\end{array}$

WBS

TPCH TASK TITLE

J $80100 A 00$ J8DIA NON-DISCRETIOHARY OPERATING COSTS

IJ8D100A01 J801B BD100 MGT ACTIVITIES

IJ8D100A03 J8010

IJ8D100BO0 J8D1E

$1 J 80100 B 02 J 8016$

$1 J 80100803 \mathrm{~J} 8011$

$1 \mathrm{J00} 100805$ J801

1J80100E01 J80 IV

IJ80 100E02 J8D IY

$1 \mathrm{~J} 80100 \mathrm{E} 03$ J8D1Y

IJ8D 100EOS J801

$1 \mathrm{~J} 80100 \mathrm{E08}$ J8D14

iJ80100G00 J8017

STAFF MOVES

PROGRAM DEVELOPMENT \& IMPLEMENTATION

EFCOG ACTIVITIES

PROFESSIOHAL SOCIETY

CONTRACTS ADMINISTRATION

MANDATORY TRAINING

\$O100 TECHNICAL TRAIHING

EDECHICAL TRAINING

DD

80150 TECHUICAL TRAINING

DO 150 TECHNICAL TRAIHING

EMERGENT HORK

TOTALS
OVERHEADS, SAFETY ANALYSIS \& ENGINEERIHG

FTE JUSTIFICATION

$$
6.3 .5
$$

FIE

1.00 INGERSOL 0.46 , HESTER 0.6

1.00

Overheads, Safety Analysis \& Engineering Page 4-14 
WHC-SP-1116 REV 0

\section{DISTRIBUTION}

\section{Number of copies}

ONSITE

6

U.S. Department of EnergyRichland Operations Office
J. B. $\mathrm{Ha} 11$
H. P. Mooers
M. R. Moreno (2)
R. P. Saget
R. E. Traister

A5-55

A5-55

A5-55

A5-54

B3-63

29

Westinghouse Hanford Company

E. P. DiVincenzo

H5-36

W. C. Floberg

B3-75

S. M. Godfrey

J. E. Holmes (2)

H5-36

L. L. Powers

N. A. Samuels

B3-62

H6-28

J. A. Sargent

H5-37

R. L. Schlosser

R. M. Suyama

G. A. Tarcza (12)

B. R. Thomas

A. J. Watts

B3-75

H4-65

H5-60

H5-36

B3-73

B3-75

Central Files (2)

L8-04

OSTI (2)

L8-07

Overheads, Safety Analysis \& Engineering Page Distr-1 
WHC-SP-1116 REV 0

This page intentionally left blank.

Overheads, Safety Analysis \& Engineering Page Distr-2 